Draft VERSion March 7, 2018

Preprint typeset using $\mathrm{LAT}_{\mathrm{E}} \mathrm{X}$ style emulateapj v. 01/23/15

\title{
A WINDOW ON THE EARLIEST STAR FORMATION: EXTREME PHOTOIONIZATION CONDITIONS OF A HIGH-IONIZATION, LOW-METALLICITY LENSED GALAXY AT Z 2 *
}

\author{
Danielle A. Berg ${ }^{1,2}$, Dawn K. Erb ${ }^{1}$, Matthew W. Auger ${ }^{3}$, Max Pettini $^{3}$, Gabriel B. Brammer ${ }^{4}$ \\ Draft version March 7, 2018
}

\begin{abstract}
We report new observations of SL2SJ021737-051329, a lens system consisting of a bright arc at $z=1.84435$, magnified $\sim 17 \times$ by a massive galaxy at $z=0.65$. SL2SJ0217 is a low-mass (M $\left.<10^{9} \mathrm{M}_{\odot}\right)$, low-metallicity $\left(\mathrm{Z} \sim 1 / 20 \mathrm{Z}_{\odot}\right)$ galaxy, with extreme star-forming conditions that produce strong nebular UV emission lines in the absence of any apparent outflows. Here we present several notable features from rest-frame UV Keck/LRIS spectroscopy: (1) Very strong narrow emission lines are measured for C IV $\lambda \lambda 1548,1550$, He II $\lambda 1640$, O III] $\lambda \lambda 1661,1666$, Si III] $\lambda \lambda 1883,1892$, and C III] $\lambda \lambda 1907,1909$. (2) Double-peaked Ly $\alpha$ emission is observed with a dominant blue peak and centered near the systemic velocity. (3) The low- and high-ionization absorption features indicate very little or no outflowing gas along the sightline to the lensed galaxy. The relative emission line strengths can be reproduced with a very high-ionization, low-metallicity starburst with binaries, with the exception of He II, which indicates an additional ionization source is needed. We rule out large contributions from AGN and shocks to the photoionization budget, suggesting that the emission features requiring the hardest radiation field likely result from extreme stellar populations that are beyond the capabilities of current models. Therefore, SL2S0217 serves as a template for the extreme conditions that are important for reionization and thought to be more common in the early Universe. Subject headings: galaxies: abundances - galaxies: evolution - galaxies: ISM - cosmology: reionization
\end{abstract}

\section{INTRODUCTION}

Determining the precise epoch and source of reionization is currently a major focus of observational cosmology. Yet, the relative contributions to ionizing radiation from stellar and nuclear activity are still uncertain (e.g., Fontanot et al. 2014). However, there is a general consensus that metal-poor, low-mass galaxies host a substantial fraction of the star formation in the high-redshift Universe and are likely the key contributors to reionization (e.g., Wise et al. 2014; Madau \& Haardt 2015). Depending on the redshift, luminosity function, intergalactic medium (IGM) clumping factor, and ionizing photon production efficiency assumed, the estimated escape fraction of ionizing radiation needed to sustain cosmic reionization ranges from $<13 \%$ up to $50 \%$ (Finkelstein et al. 2012). Therefore, detailed studies of these chem-

* THIS WORK WAS SUPPORTED BY A NASA KECK PI DATA AWARD, ADMINISTERED BY THE NASA EXOPLANET SCIENCE INSTITUTE. DATA PRESENTED HEREIN WERE OBTAINED AT THE W. M. KECK OBSERVATORY FROM TELESCOPE TIME ALLOCATED TO THE NATIONAL AERONAUTICS AND SPACE ADMINISTRATION THROUGH THE AGENCY'S SCIENTIFIC PARTNERSHIP WITH THE CALIFORNIA INSTITUTE OF TECHNOLOGY AND THE UNIVERSITY OF CALIFORNIA. THE OBSERVATORY WAS MADE POSSIBLE BY THE GENEROUS FINANCIAL SUPPORT OF THE W. M. KECK FOUNDATION.

${ }^{1}$ Center for Gravitation, Cosmology and Astrophysics, Department of Physics, University of Wisconsin Milwaukee, 3135 N. Maryland Avenue, Milwaukee, WI 53211; bergda@uwm.edu; erbd@uwm.edu

2 Department of Astronomy, The Ohio State University, $140 \mathrm{~W}$. 18th Avenue, Columbus, OH 43202; berg.249@osu.edu

${ }^{3}$ Institute of Astronomy, Madingley Road, Cambridge, CB3 0HA, United Kingdom; mauger@ast.cam.ac.uk; pettini@ast.cam.ac.uk

${ }^{4}$ Space Telescope Science Institute, 3700 San Martin Dr, Baltimore, MD 21211; brammer@stsci.edu ically unevolved galaxies at early epochs are necessary to probe the initial stages of galaxy evolution and assess whether the physical conditions allow sufficient Ly continuum radiation to escape.

Surveys of galaxies at $2 \lesssim z \lesssim 4$ reveal a rich diversity of galaxy properties and mark a critical stage of galaxy evolution at which the peak of both star formation and black hole accretion activity occur (see Shapley (2011) for a review). These surveys have provided us with a broad understanding of how galaxies assemble and evolve, but the spatial and spectral limitations inherent in observing faint, distant objects mean that many of the physical processes regulating this dynamic evolution are poorly constrained. Clearly, a more detailed understanding of this key epoch is needed.

Until recently, very few high-redshift galaxies had been observed spectroscopically in detail, as they are generally too faint to obtain high signal-to-noise observations with even $8-10 \mathrm{~m}$ class telescopes. Previously, this challenge has been overcome by building composite spectra of highredshift galaxies (e.g., Steidel et al. 2001; Shapley et al. 2003 Steidel et al. 2016, Rigby et al. 2017), the properties of which generally indicate the sources are young $\left(\sim 10^{8} \mathrm{yrs}\right)$, metal-poor $\left(\mathrm{Z}_{n e b}<0.5 \mathrm{Z}_{\odot}\right)$ galaxies with high specific star formation rates and strong outflows of ionized gas. Currently, our most detailed information on the physical conditions in galaxies at moderate redshift comes from careful, high $\mathrm{S} / \mathrm{N}$ spectroscopic studies of gravitationally lensed objects (e.g., Pettini et al. 2000, 2002b; Fosbury et al.|2003; Hainline et al.|2009, Quider et al. 2009; Yuan \& Kewley 2009, Quider et al. 2010; Dessauges-Zavadsky et al. |2010; Rigby et al. 2011; Jones et al. 2013 Amorín et al. 2014, Vanzella et al. 2016). However, samples of such galaxies are necessarily obtained one at a time, and so remain small and do 
not yet adequately sample the diversity among high redshift galaxy populations. In particular, there are few detailed studies of low mass, low metallicity $\left(\mathrm{Z}_{n e b}<0.1 \mathrm{Z}_{\odot}\right)$ sources, and only a handful of targets with direct determinations of their physical conditions such as electron temperature, density, and gas phase oxygen abundance (e.g., Hainline et al. 2009, Bayliss et al. 2014; Christensen et al. 2014).

In order to understand how the physical conditions in galaxies have evolved over cosmic time, we need detailed studies of galaxies at early epochs that have undergone little chemical evolution in comparison with nearby counterparts. The Strong Lensing Legacy Survey $\sqrt{\mathrm{Tu}}$ et al. 2009) has discovered several such targets, including the $z \sim 1.85$ gravitationally lensed arc SL2SJ02170513, hereafter SL2S0217. Low-resolution rest-frame UV and optical spectra of this arc display very strong, highionization nebular emission lines, suggesting SL2S0217 is both very metal poor and highly ionized (Tu et al. 2009 Brammer et al. 2012).

In preparation for the next frontier of high-redshift studies made possible by the forthcoming generation of space-based (i.e., JWST) and 30m ground-based observatories (i.e., GMT, ELT, and TMT), we seek to understand the conditions producing the extreme UV emission lines seen in SL2S0217 and even higher-redshift sources. Because the intergalactic medium becomes increasingly neutral at $z>6$, Ly $\alpha$ emission, which is typically the strongest emission feature in UV spectra of high redshift Ly $\alpha$-emitting galaxies ${ }^{2}$, is increasingly scattered and therefore suppressed (Stark 2016). Instead, the next strongest UV emission lines, such as C III] must be used to spectroscopically confirm redshifts. Additionally, these lines will provide useful constraints on the physical properties of the emitting source and may be used as important diagnostics for characterizing high-redshift galaxies. Recently, these strong UV nebular emission lines have been observed in high redshift $(z>6)$ galaxies (e.g., Stark et al. 2015, Stark 2016), indicative of low-metallicity and high-ionization (e.g., Erb et al. 2010 Stark et al. 2014). Because high-ionization targets may also have large escape fractions of $\mathrm{H}$-ionizing photons (e.g., Brinchmann et al. 2008; Jaskot \& Oey 2013; Stark et al. 2014), SL2S0217 is a good candidate for studying the chemically unevolved, extreme physical conditions that are expected in the early galaxies responsible for reionizing the Universe.

Here we present the analysis of new Keck LRIS spectroscopy of SL2S0217. Section 2 describes the previous observations and derived properties of SL2S0217 presented in Brammer et al. (2012). In Section 3 we present our analysis of the existing photometry and an improved lensing model for SL2S0217. The spectral observing plan and data reduction for the new LRIS spectrum are laid out in Section 4. We examine the remarkable features of the resulting rest-frame UV spectrum in Section 5, paying particular attention to the nebular emission lines ( $\S 5.2$, the double-peaked Ly $\alpha$ profile ( $\S 5.3$ ), and the interstellar absorption line profiles $(\S 5.4)$. The physical conditions of the gas and chemical abundances of oxygen, carbon, nitrogen, and silicon are estimated from the nebular emission lines in Section 7. Finally, in Section 8, we attempt to reproduce our spectra and constrain the ionization source by inspecting a large grid of photoioniza-
TABLE 1

Observations OF SL2SJ0217-0513

\begin{tabular}{|c|c|}
\hline Observing Program & Band / Wavelength \\
\hline $\begin{array}{l}\text { PHOTOMETRY: } \\
\text { HST CANDELS }\end{array}$ & $\begin{array}{l}\text { ACS F606W (V), F814W (i) } \\
\text { WFC3 F125W (J), F160W (H) }\end{array}$ \\
\hline 3D-HST & F140W ( $\left.\mathrm{H}_{\text {wide }}\right)$ \\
\hline Spitzer & MIPS $24 \mu \mathrm{m}(\mathrm{mJy})$ \\
\hline $\operatorname{HST} \operatorname{Ly} \alpha$ & WFC3 F343N, F390M \\
\hline SPECTROSCOPY: & \\
\hline 3D-HST Optical & WFC3 G141; $1.10-1.65 \mu \mathrm{m}$ \\
\hline Keck/LRIS UV & $600 / 4000 ; \sim 3100-9000 \AA$ \\
\hline
\end{tabular}

tion models, considering contributions from stars, shocks $(\S 8.3 .1)$, and active galactic nuclei (AGN; $\S 8.3 .2$. The photoionization budget is discussed further in the context of the strong C IV $(\S 8.4 .1)$ and He II $(\S 8.4 .2)$ emission. Throughout this paper we adopt a flat FRW metric with $\Omega_{\mathrm{m}}=0.3, \Omega_{\Lambda}=0.7$, and $\mathrm{H}_{0}=70 \mathrm{~km} \mathrm{~s}^{-1} \mathrm{Mpc}^{-1}$, and the solar metallicity scale of Asplund et al. (2009), where $12+\log (\mathrm{O} / \mathrm{H})_{\odot}=8.69$.

\section{GALAXY PROPERTIES AND PAST OBSERVATIONS}

SL2SJ0217 is a gravitationally lensed galaxy at a redshift $z \sim 1.85$ that is lensed by a massive galaxy at $z=$ 0.6459 as discovered by the Strong Lensing Legacy Survey (Tu et al. 2009). The general properties of SL2S0217 are well characterized due to its location within both the HST CANDELS imaging survey (ACS F606W and F814W and WFC3 F125W and F160W Grogin et al. 2011; Koekemoer et al. 2011), and 3D-HST spectroscopic survey (WFC3 G141 grism spectroscopy; Brammer et al. 2012, hereafter B12). Additionally, a low-resolution Keck LRIS spectrum ( $\mathrm{Tu}$ et al. 2009) exists for the arc. All existing imaging and spectral observations for SL2S0217 are summarized in Table 1 .

B12 report that the arc is very blue with a UV slope of $\beta=-1.7 \pm 0.2\left(f_{\lambda} \propto \lambda^{\beta}\right)$ for $\lambda_{\text {rest }}=2100-2800$ $\AA$, but is rather red in the F160W band due to strong [O III] $\lambda \lambda 4959,5007$ emission. The low resolution WFC3 G141 grism spectrum of the lens system presented in B12 shows extremely high equivalent width rest-frame optical emission lines that are atypical of $z \sim 0$ starforming galaxies (namely, rest-frame $\mathrm{EW}(\mathrm{H} \beta)=1470 \AA$ and $\mathrm{EW}([\mathrm{O}$ III $] \lambda \lambda 4959,5007)=5690 \AA)$. The emission lines are all spatially extended, indicating that strong nebular emission is coming from multiple clumps along the arc. From their analysis of this grism spectrum and modeling of the broadband spectral energy distribution, B12 concluded that the arc is a young, low-mass, lowmetallicity $(12+\log (\mathrm{O} / \mathrm{H}) \sim 7.5)$ star-bursting (specific star formation rate, sSFR, $\sim 100 \mathrm{Gyr}^{-1}$ ) galaxy, with similar characteristics as local blue compact dwarf galaxies. SL2S0217 is thus one of the lowest metallicity starforming galaxies yet identified at $z>1$. Derived quanti-

\footnotetext{
2 Typical, moderately massive $z \sim 2-3$ galaxies actually have
} net Ly $\alpha$ absorption. 
TABLE 2

Properties of SL2SJ0217-0513

\begin{tabular}{|c|c|}
\hline Parameter & Value \\
\hline $\begin{array}{l}\text { R.A. } \\
\text { Dec. } \\
z\end{array}$ & $\begin{array}{c}02: 17: 37.237 \\
-05: 13: 29.78 \\
1.84435 \pm 0.00066\end{array}$ \\
\hline \multicolumn{2}{|c|}{ From Brammer et al. (2012): } \\
\hline $\begin{array}{l}\log (\text { age } / \mathrm{yr}) \\
\mu_{\star} \\
\mu^{\prime} \\
A_{V}(\text { continuum }) \\
\log \left(\mu \cdot M / M_{\odot}\right) \\
\beta(2000-2800 \AA) \\
\mu \cdot \mathrm{SFR}_{\mathrm{H} \beta}\left(M_{\odot} \mathrm{yr}^{-1}\right) \\
\sqrt{\mu^{\prime}} \cdot r_{e}\end{array}$ & $\begin{aligned} 7.2 & \pm 0.2 \\
25 & \pm 1^{a} \\
\sim & 1.4^{a} \\
0.09 & \pm 0.15 \\
9.5 & \pm 0.1 \\
-1.7 & \pm 0.2 \\
390 & \pm 9 \\
350 \mathrm{pc} & \end{aligned}$ \\
\hline \multicolumn{2}{|c|}{ Using Updated Lens Model: } \\
\hline $\begin{array}{l}\mu_{t o t} \\
\mu_{\text {eff }} \\
\log \left(M_{\star} / M_{\odot}\right) \\
\operatorname{SFR}\left(M_{\odot} \mathrm{yr}^{-1}\right)^{b}\end{array}$ & $\begin{array}{c}17.3 \pm 1.2 \\
19 \pm 1.5 \\
8.26 \pm 0.10 \\
22.5 \pm 1.6\end{array}$ \\
\hline $\begin{array}{l}\text { NOTE. - Observed ar } \\
\text { for SL2SJ0217-0513. T } \\
\text { (Koekemoer et al. } 2011 \\
\text { et al. 2006) photometry } \\
\text { ties derived from the 3D- } \\
\text { and SED fit, are taken } \\
\text { et al. (2012). Updated pa } \\
\text { ing the lensing model fro } \\
\text { listed. RA and Dec spe } \\
\text { ter in units of hours, m } \\
\text { and degrees, arcminutes, } \\
\text { spectively. } \\
a \mu_{\star} \text { is the relative magn } \\
\text { integrated arc and the cou } \\
\text { brightness magnification } \\
\text { The total lens magnificat } \\
{ }^{b} \text { Determined from the } \mathrm{H}\end{array}$ & $\begin{array}{l}\mathrm{d} \text { derived quantities } \\
\text { e HST CANDELS } \\
\text { and MIPS (Labbé } \\
\text { as well as proper- } \\
I S T \text { grism spectrum } \\
\text { the from Brammer } \\
\text { ameters incorporat- } \\
\text { n this work are also } \\
\text { ify the optical cen- } \\
\text { autes, and seconds, } \\
\text { and arcseconds re- } \\
\text { ication between the } \\
\text { nter image. } \mu^{\prime} \text { is the } \\
\text { f the counter image. } \\
\text { on is } \mu=\mu_{\star} \cdot \mu^{\prime} \text {. } \\
3 \text { line luminosity. }\end{array}$ \\
\hline
\end{tabular}

ties from B12 are given in the top of Table 2

\section{IMPROVED PHOTOMETRY AND LENSING MODEL}

Using the available 5-band $H S T$ imaging, we quantify the resolved photometric properties of SL2S0217. These data include optical F606W and F814W images observed with ACS/WFC (4,856 s and 11,300 s respectively) and near infrared F125W, F140W, and F160W images observed with WFC3/IR $(1,912 \mathrm{~s}, 812 \mathrm{~s}$, and 3,512 s respectively). All of the data were reduced with the AstroDrizzle package (Fruchter et al. 2010) and were resampled to a pixel scale of $0.05^{\prime \prime}$. The absolute astrometry from the telescope differs between the different bands, but we ensured that each band was well registered by matching the apparent coordinates of the central lensing galaxy in the output resampled images. We used an unsaturated bright star $21^{\prime \prime}$ from the lens as a model for the PSF in our subsequent surface brightness and lens modeling.

The surface brightness distribution of the foreground lensing galaxy overlaps with the lensed arc features, and we therefore modeled and removed this light from each image using the following process. First, we manually masked several interloping objects that lie both within and around the lens system. Next, we constructed a mask for the arc and counter image and simultaneously fit the foreground lensing galaxy light distribution in each band with a model that allowed three concentric Sérsic profiles. As a test of this procedure, we have also fit the foreground light in the $\mathrm{F} 606 \mathrm{~W}$ band while simultaneously modeling the light of the background source and found consistent results. Finally, the foreground surface brightness model was subtracted from the data to give an uncontaminated view of the background source structure.

The structure of the bright, lensed arc indicates that the background galaxy has a complex morphology. We therefore used the adaptive pixellated source modeling technique described in Vegetti \& Koopmans (2009) to delens and 'reconstruct' the lensed source. The intrinsic source surface brightness distribution was described on an irregular grid of pixels that approximately follows the magnification of the lens, with a PSF-deconvolved intensity at each pixel determined from the lens mass model and observed data. We modeled the lensing potential as an elliptical powerlaw mass distribution with an empirical external shear, and constrained it with the F606W data, which have excellent spatial resolution and $\mathrm{S} / \mathrm{N}$. The reconstructed source surface brightness distribution is shown in the right panel of Figure 1 .

The total observed (magnified) magnitudes are given in Table 3, with systematics-dominated uncertainties of $\sim 0.03$ magnitudes. While the increased freedom in our lens model generally increases the uncertainties on the magnification, our flexible source model allows a better fit to the data, decreasing the systematic uncertainty. Therefore, the lens modeling yields a total magnification of $\mu=17.3 \pm 1.2$, significantly lower than the value of $\sim 35$ determined by Cooray et al. (2011) and reported in B12. This discrepancy in magnification is largely driven by the choice of source model. In our analysis, we find that there is a significant amount of low-surface-brightness (and low-magnification) flux that is not encapsulated by the Cooray et al. (2011) sources, and for that reason the magnification (observed light)/(modeled source light) is larger for their model. ${ }^{3}$

This low-surface-brightness flux is readily seen in the bluer filters, where the light from the lensing galaxy is less prominent, as extensions of the bright arc immediately north and south of the lensing galaxy. Our flexible source model allows magnification to vary across the source, and results in the intrinsically higher-surfacebrightness regions (highlighted in the right panel of Figure 1) having somewhat higher magnifications of $\sim 25$. In other words, there is clearly differential magnification across the broad-band image of the source. We simulated slit losses of $\sim 10 \%$ by convolving the light profile from the HST F606W image with the seeing disk and integrating through the $1^{\prime \prime}$ slit of the LRIS spectrum. Accounting for the lensing magnification and applying the slit loss correction, we find that the flux within the spectroscopic aperture has an effective mean amplification of $19 \pm 1.5$.

As in B12, we find significant broad-band color differ-

\footnotetext{
3 Cooray et al. (2011) used two exponential profiles for the source and a singular isothermal elliptical (SIE) lens, perturbed by a distant singular isothermal sphere (SIS) halo. This model fixes the external shear field and the radial distribution. The subsequent lack of structure in the source surface brightness model results in significant residuals (see Figure 2(h) of Cooray et al. (2011)).
} 

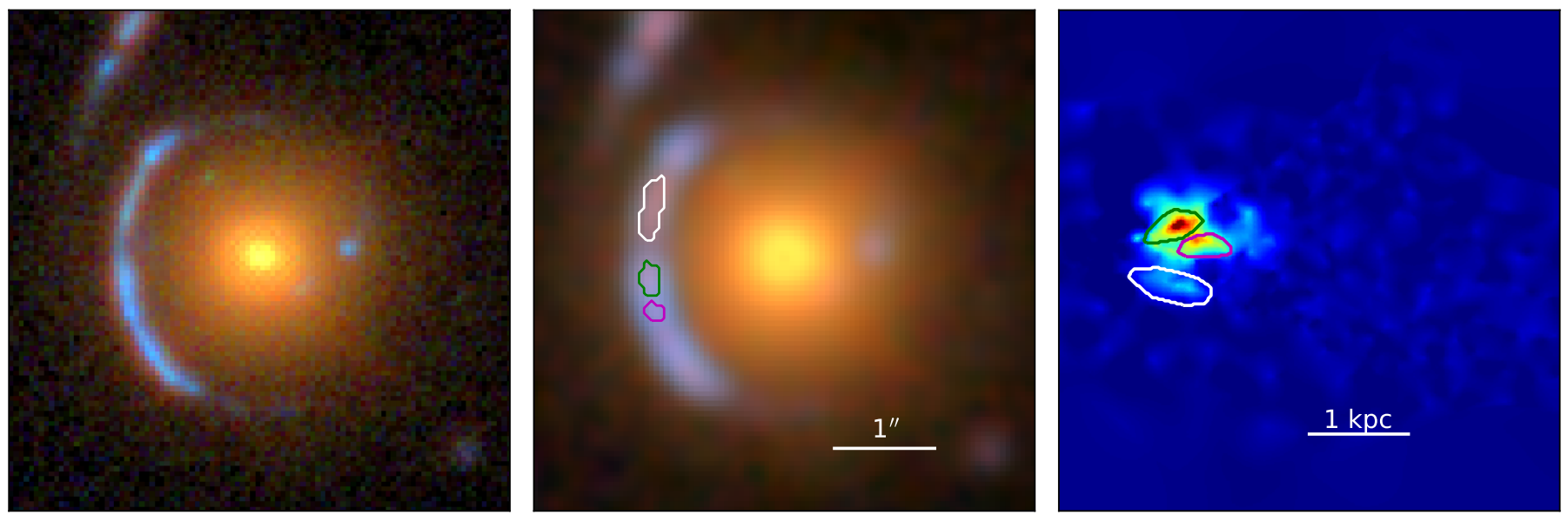

Fig. 1.- (Left) HST F606W, F814W, and F125W image of the lens. (Middle) Same, but with the resolution degraded to the resolution of the F160W image to perform aperture photometry within the shown apertures. (Right) Reconstructed source in the F606W filter, showing the intrinsic three regions corresponding to the apertures in the middle panel.

TABLE 3

Lens Model Photometry

\begin{tabular}{lccc}
\hline \hline \multicolumn{1}{c}{ Parameter } & \multicolumn{3}{c}{ Region } \\
\hline & \multicolumn{3}{c}{ Total } \\
F606W & \multicolumn{3}{c}{22.15} \\
F814W & 22.03 & \\
F125W & 21.80 & \\
F140W & \multicolumn{3}{c}{21.14} \\
F160W & \multicolumn{3}{c}{20.98} \\
\hline & Magenta & Green & White \\
\cline { 2 - 4 } F606W-F160W & $1.03 \pm 0.10$ & $1.35 \pm 0.19$ & $1.79 \pm 0.27$ \\
F814W-F160W & $1.01 \pm 0.10$ & $1.24 \pm 0.18$ & $1.53 \pm 0.25$ \\
F125W-F160W & $0.79 \pm 0.08$ & $0.93 \pm 0.14$ & $1.01 \pm 0.21$ \\
F140W-F160W & $0.25 \pm 0.08$ & $0.28 \pm 0.13$ & $0.26 \pm 0.20$
\end{tabular}

Note. - Top: The total observed (magnified) magnitudes, with systematics-dominated uncertainties of $\sim 0.03$ magnitudes. Bottom: The colors measured for the magenta, green, and white apertures show significant broadband color differences across the galaxy.

ences across the galaxy. To quantify this, we convolved each of the four bluer images with a gaussian filter to match the resolution of the $\mathrm{F} 160 \mathrm{~W}$ image and determined aperture colors within the three regions shown in Figure 1. The colors measured for the magenta, green, and white apertures are listed in Table 3 . The white region, which is clearly redder, is also much lower surface brightness and lower magnification than the other regions, and so contributes less to the observed LRIS spectrum. Thus, the UV spectrum of SL2S0217 is largely produced by the neighboring magenta and green starforming regions.

\section{NEW KECK/LRIS SPECTROSCOPIC OBSERVATIONS AND DATA REDUCTION}

\subsection{Observations}

We obtained spectra of SL2S0217 using the Low Resolution Imaging Spectrograph (LRIS; Oke et al. 1995) on the Keck I telescope on the UT date of 2015 October 12. The $600 / 4000$ grism and $600 / 7500$ grating were used with the blue and red detectors respectively, with the dichroic at $560 \mathrm{~nm}$, resulting in an observed-wavelength coverage of approximately $3100-9000 \AA$ and an average resolution at full width half maximum (FWHM) of roughly $4.0 \AA\left(275 \mathrm{~km} \mathrm{~s}^{-1}\right)$ in the blue and $4.7 \AA(190 \mathrm{~km}$ $\left.\mathrm{s}^{-1}\right)$ in the red. This corresponds to a rest-wavelength coverage of approximately $1100-3000 \AA$ and resolutions of 1.4 (1.7) $\AA$ in the blue (red).

Internal and twilight calibration flats were obtained at the beginning of the night to account for differences between the chip illumination patterns on the sky and the internal lamps. Two standard stars, Feige 24 and Feige 110, with spectral energy distributions peaking in the blue were observed over a range of hour angles throughout the night, allowing the flux calibration to be determined as a function of airmass. SL2S0217 was observed for $13 \times 1800 \mathrm{~s}$ or 6.5 hours in clear, dark conditions with $0.7-0.8^{\prime \prime}$ seeing, over an airmass range of $1.0-1.7$. A slit of $1.0^{\prime \prime} \times 168^{\prime \prime}$ at an angle of $3^{\circ}$ was chosen in order to encompass the maximum flux from the arc, as shown in Figure 2. HgNeArCdZn arc lamps were observed after every other exposure to mitigate the effects of instrument flexure in the wavelength calibration.

\subsection{Spectra Reduction}

The LRIS spectra were processed using ISPEC2D (Moustakas \& Kennicutt 2006), a long-slit spectroscopy data reduction package written in IDL. Master sky and internal flats were constructed by taking the median at each pixel after normalizing the counts in the individual images. These calibration files were then used to bias-subtract, flat-field, and illumination-correct the raw science data frames. Misalignment between the trace of the light in the dispersion direction and the orientation of the CCD detector was rectified via the mean trace of the standard stars, providing alignment to within a pixel across the detector. A median sky subtraction was performed at each column along the dispersion, followed by a wavelength calibration applied from the $\mathrm{HgNeArCdZn}$ comparison lamps taken at the nearest airmass. The onedimensional spectra were produced using a box-car aperture that encompassed roughly $99 \%$ of the light in the Ly $\alpha$ feature. We note that while a second lensed source is with in the top part of the slit (see Figure 2), it is 


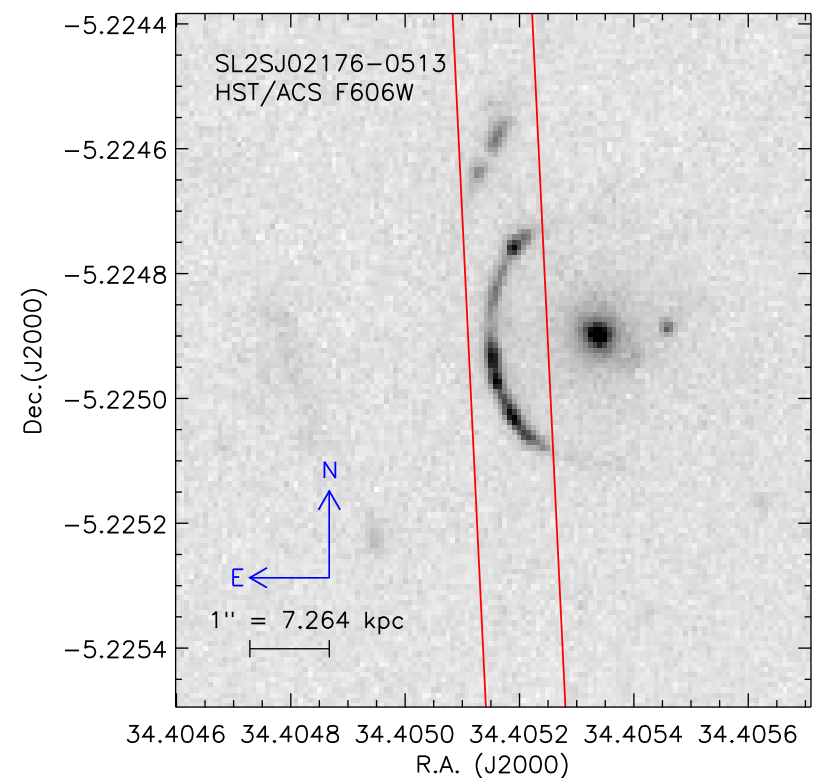

Fig. 2.- CANDELS HST ACS F606W image of SL2SJ02170513. The $1.0^{\prime \prime}$ LRIS slit is overlaid in red, demonstrating that the majority of the arc light was captured in the slit. A second lensed source is visible in the top part of the slit, but it is spatially distinct (both along the slit and in redshift) and so does not affect the spectrum of SL2SJ0217-0513.

distinct spatially, as well as in redshift $(z \sim 2.32$; Cooray et al. 2011), and so does not affect the extracted spectrum of SL2SJ0217. Individual exposures were then flux calibrated using the sensitivity curve derived from the standard star observations taken throughout the night. Finally, the 13 sub-exposures were median combined, eliminating cosmic rays in the process.

The resulting 1D spectra were then compared as a check on the flux calibration: the overlapping regions of flux-calibrated blue and red spectra were found to be in good agreement within the dispersion of their continua. We then checked the absolute flux calibration of the combined blue+red LRIS spectrum using newly obtained HST images in the WFC3 F390M (covering restframe $\sim 1300-1400 \AA$ ) and F343N (covering the Ly $\alpha$ emission line) filters; these images will be presented in a future paper. We used the PYSYNPHOT package in Python to predict the F390M AB magnitude from the spectrum, and then scaled the spectrum to match the observed F390M AB magnitude $(22.68 \pm 0.07$; Erb et al. 2018, in prep.). Note that we used the bluest possible continuum band so that contamination of the lens is negligible. The resulting 1D spectrum and error spectrum are shown in Figure 3. Regions of strong sky line emission are shaded grey. Potential emission and absorption features are labeled accordingly.

\section{THE REST-FRAME UV SPECTRUM}

The blue portion of the rest-frame UV LRIS spectrum of SL2S0217 is shown in Figure 4, where the middle and bottom panels are zoomed in on the vertical scale to highlight the numerous absorption and emission features observed. In comparison we plot the composite spectrum of $\sim 1,000 z \sim 2$ galaxies from Erb et al. (2010), and note two defining properties of the SL2S0217 spectrum: 1) The interstellar absorption features typical of $z \sim 2$
TABLE 4

Emission Line Redshift Determinations

\begin{tabular}{cccc}
\hline \hline Ion & $\lambda_{\text {lab }}(\AA)^{a}$ & $\lambda_{\text {obs }}(\AA)$ & $\mathrm{z}$ \\
\hline O III] & 1660.81 & 4723.97 & 1.84438 \\
O III] & 1666.15 & 4739.03 & 1.84430 \\
Si III] & 1882.71 & 5355.23 & 1.84443 \\
Si III] & 1892.03 & 5381.48 & 1.84429 \\
{$[$ C III] } & 1906.68 & 5423.25 & 1.84434 \\
C III] & 1908.73 & 5429.13 & 1.84437 \\
\hline
\end{tabular}

Average: $\quad 1.84435 \pm 0.00066$

Note. - Systemic redshift of SL2S0217 calculated from the strong nebular emission lines in the LRIS spectrum.

a Vacuum wavelengths.

galaxies appear to have velocity profiles that are roughly 3 times narrower in SL2S0217, and 2) significant, highionization nebular emission is present in SL2S0217 that is atypical of $z \sim 2$ galaxies. We further discuss some of the most striking features of the LRIS spectrum below.

\subsection{Source Redshift}

In order to analyze the velocity structure of the absorption and emission features seen in the SL2S0217 UV spectrum, it is necessary to first establish a reliable determination of the systemic redshift as the reference velocity zero point. Tu et al. (2009) used all of the strong emission lines present in their low-resolution Keck/LRIS spectrum (Ly $\alpha, \mathrm{C}$ IV, He II, O III], and C III]) to estimate $z_{\text {arc }}=1.84691 \pm 0.0024$. However, our LRIS spectrum shows that the Ly $\alpha$ emission for SL2S0217 is double peaked (see Figure 6). Additionally, both the He II and C IV emission can have complex profiles complicated by combinations of stellar and nebular contributions. Therefore, we choose not to use these lines in our redshift measurement. With the higher resolution of our LRIS spectrum, we are able to use the O III] $\lambda \lambda 1660,1666$, Si III] $\lambda \lambda 1883,1892$, and C III] $\lambda \lambda 1907,1909$ emission lines for our systemic redshift determination.

The line centers used to calculate the redshift are given in Table 4, resulting in a systemic redshift of $z_{\text {sys }}=$ $1.84435 \pm 0.00066$ for SL2S0217. Since this value is within the uncertainty of the redshift measured by Tu et al. (2009), the line centers of the observed Ly $\alpha$, He II, and C IV profiles must lie close to the systemic velocity.

\subsection{Nebular Emission Lines}

The rest-frame UV spectrum of SL2S0217 contains numerous emission line features that are sensitive to the ionizing stellar population, physical properties of the emitting gas, and kinematics of outflows. In particular, SL2S0217 shows double-peaked Ly $\alpha$ emission and exceptionally strong, nebular-like emission from highionization species such as C IV $\lambda \lambda 1548,1550$, He II $\lambda 1640, \mathrm{O}$ III] $\lambda \lambda 1661,1666, \mathrm{Si}$ III] $\lambda \lambda 1883,1892$, and C III] $\lambda \lambda 1907,1909$, with additional weak detections of the lowionization [O III] $\lambda 2322$ and C II] $\lambda \lambda \lambda 2325,2327,2328$ lines. While UV emission lines have rarely been observed at strengths comparable to SL2S0217 in starforming galaxies at any epoch, these features are especially uncommon for the typically older, more massive star-forming galaxies studied at $z \sim 2$ (see, e.g., Jones 


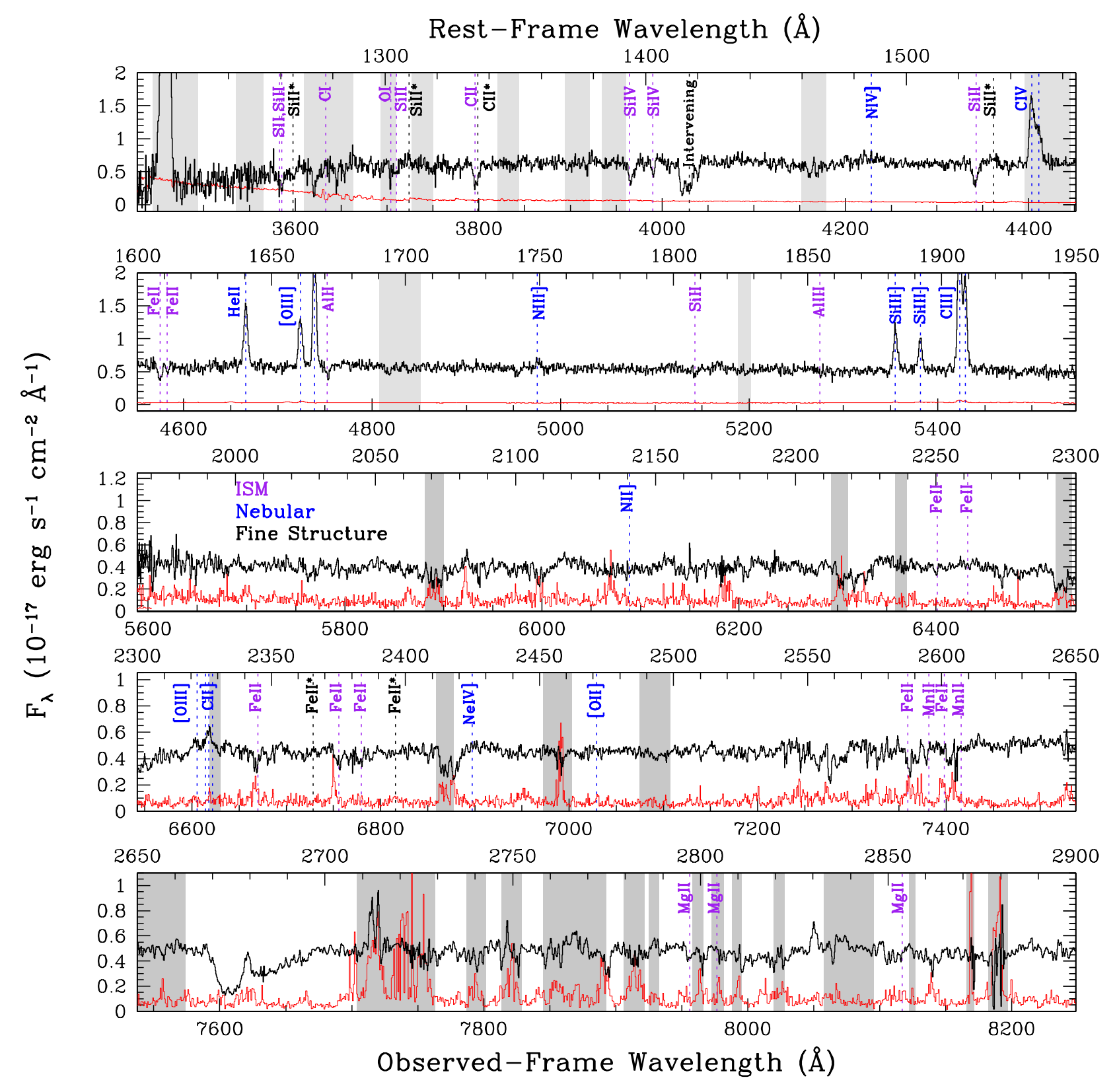

Fig. 3.- The absorption and emission features observed in the LRIS spectra of SL2S0217, with the error spectrum shown in red. Regions of strong sky line emission are shaded grey. Note that the sky features in the red spectrum are stronger than in the blue, and so are shaded darker gray accordingly. The locations of nebular emission lines are identified with dashed blue lines. Absorption features due to the interstellar medium are identified by dashed purple lines, and corresponding fine structure emission lines are labeled in black. Not all labels correspond to detections, but identify the location of features discussed in the text.

et al. 2012, Reddy et al. 2012, Whitaker et al. 2012). Figures 3 and 4 highlight these atypical emission features of SL2S0217. However, extreme emission-line features may be more common at higher redshifts where we expect to find harder radiation fields associated with less evolved, more metal-poor galaxies. Therefore, the magnified emission-line spectrum of SL2S0217 provides a unique window to examine the conditions driving powerful photoionization in distant galaxies.

To characterize the emission line spectrum, all emission line strengths for our LRIS spectrum were measured using the SPLOT routine within IRAF ${ }^{4}$. With the exceptions of Ly $\alpha, \mathrm{C}$ IV, and He II which have multiple compo- nents, groups of nearby lines were constrained to a single Gaussian FWHM and a single shift in wavelength from vacuum when possible ${ }^{5}$. Note that the nebular emission lines are unresolved, and so subsequent discussions of line widths do not account for the instrumental resolution. Following B12, who found that the $\mathrm{H} \gamma / \mathrm{H} \beta$ ratio from the grism spectrum was consistent with no reddening, we ignored the reddening from dust and simply corrected the line fluxes for the galactic extinction along the line of sight to SL2S0217 $(E(B-V)=0.0194 ;$ Schlafly \& Finkbeiner 2011) ${ }^{6}$ using the Cardelli et al. (1989) reddening law. The adopted emission line fits are depicted in Figure 5, with with the resulting line strengths and 


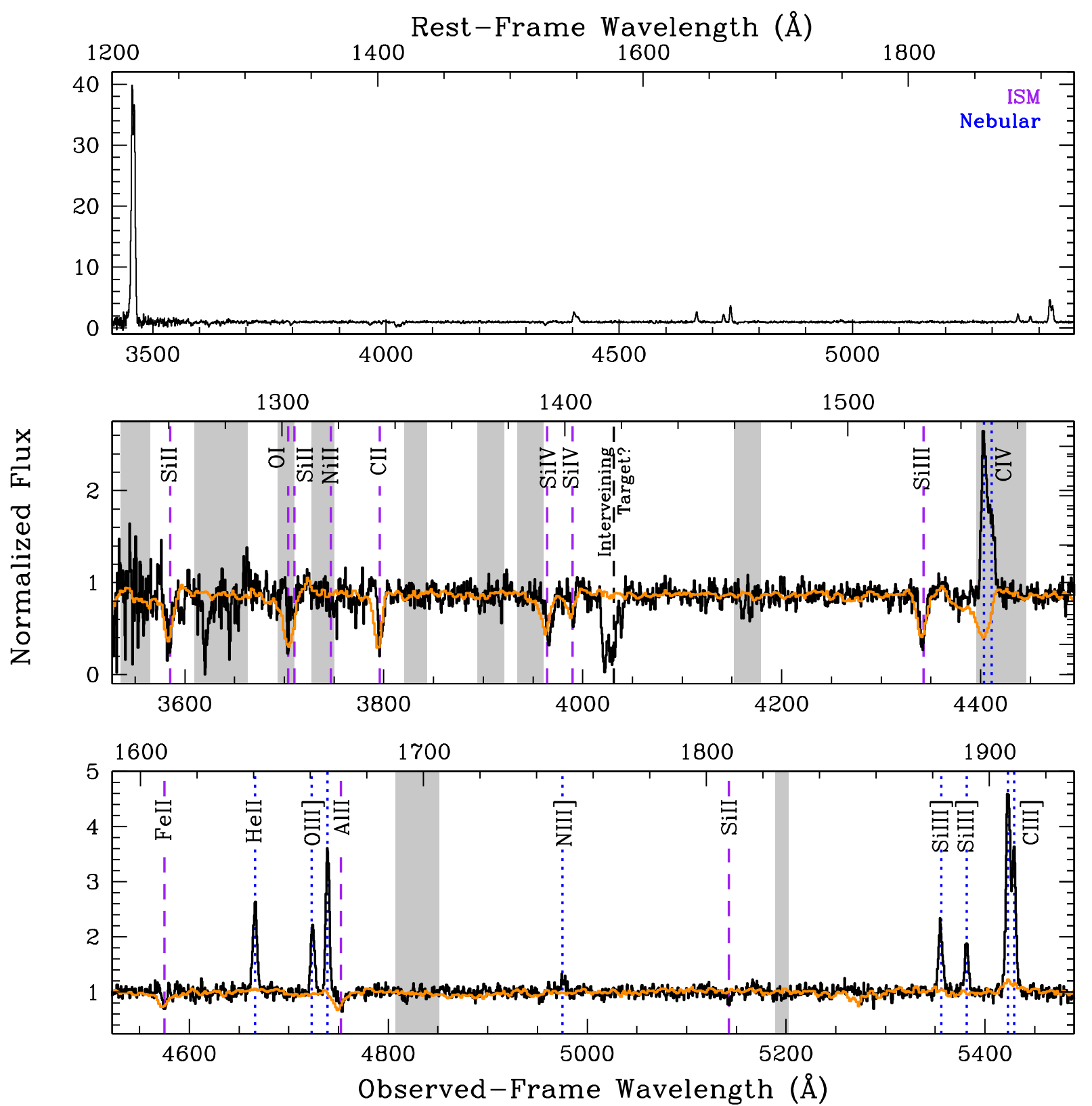

FIG. 4.- Blue arm of the Keck LRIS spectrum of SL2S0217, where regions of significant sky contamination are designated by the grey shading. The top panel shows the full extent of the spectrum, whereas the bottom two panels are zoomed in to depict the numerous interstellar medium (ISM) absorption and nebular emission features. The $z \sim 2$ composite spectrum from Erb et al. (2010) is over plotted in orange, demonstrating the staggering difference in the strength of the emission features and widths of the absorption teatures between the two spectra.

intensities given in Table 5 . We examine a few of the interesting spectral features of SL2S0217 individually in the next sections and in the discussion (Section 8).

\subsubsection{Blended Emission Features}

Neither the components of the double Ly $\alpha$ emission feature nor the individual lines of the C III] $\lambda \lambda 1907,1909$ or C IV $\lambda \lambda 1548,1550$ doublets are resolved, but are still well fit by two blended Gaussians. C IV and He II are further complicated by the potential for their profiles to be modified by multiple components. In the case of He II, which can have both nebular and stellar emission, we used a two-component fit with narrow and wide profiles respectively. Both single and multi-component fits to the He II profile are demonstrated in Figure 5. While the two-component model may offer a more visually appeasing fit to the He II profile, it does not significantly reduce the fit residuals, and so the significance of the wide $\left(\sim 700 \mathrm{~km} \mathrm{~s}^{-1}\right)$, stellar component is difficult to assess with the current $\mathrm{S} / \mathrm{N}$ and resolution. We therefore suggest that the reported fits are upper/lower limits for the stellar/nebular components. Regardless of the correct fit, the He II emission in SL2S0217 is clearly dominated by a narrow component whose width is consistent with 


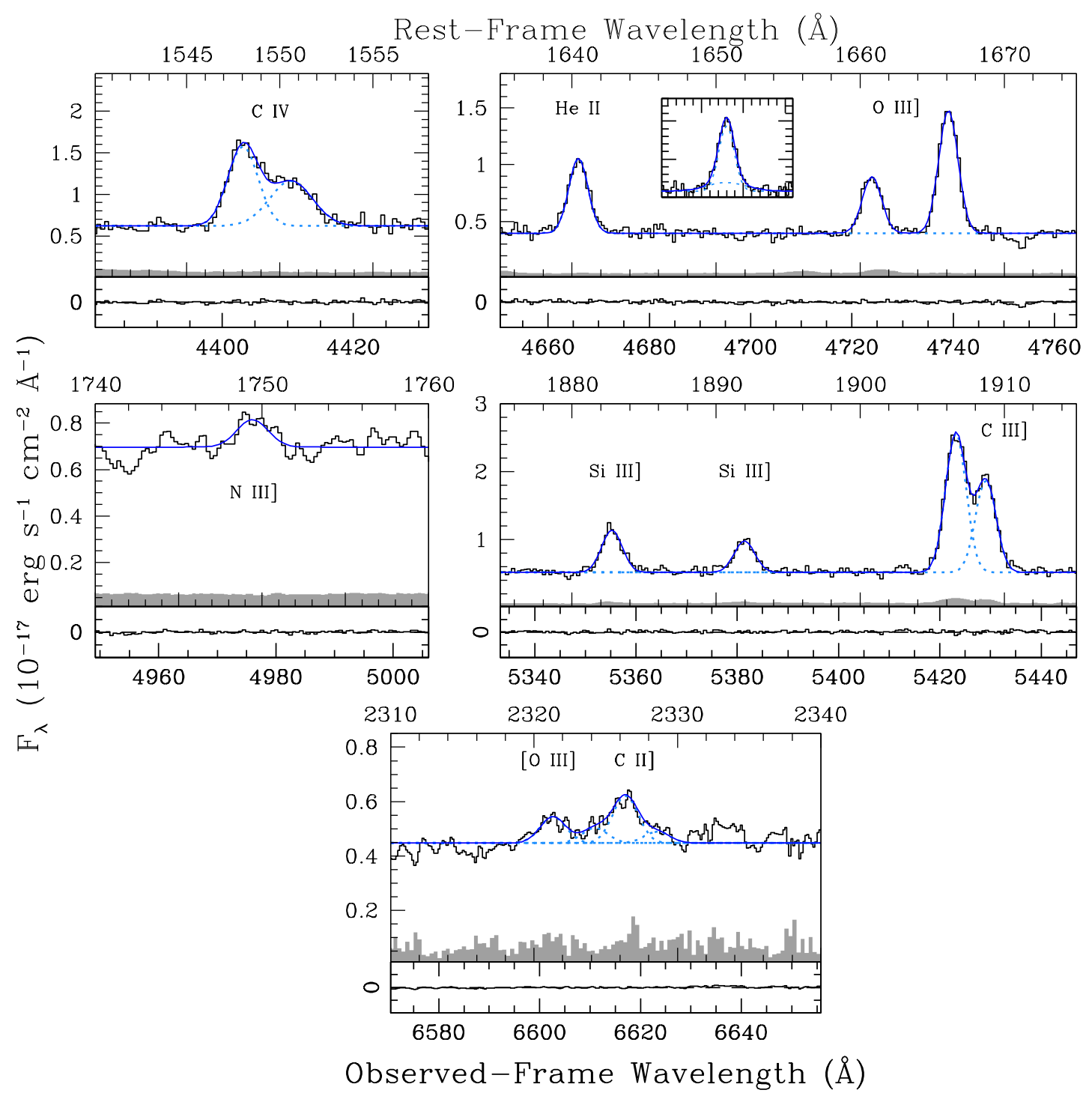

FIG. 5.- The unusually strong nebular emission lines of SL2S0217. Lines are fit by Gaussian profiles (blue), with FWHMs and wavelength shifts tied together for nearby lines. When multiple components are fit to a single blended structure, the components are shown by dashed light blue lines. In the case of He II, we show both a single Gaussian fit and a two-component fit (inset window) allowing for narrow $(\sim 280$ $\left.\mathrm{km} \mathrm{s}^{-1}\right)$ nebular and broad $\left(\sim 710 \mathrm{~km} \mathrm{~s}^{-1}\right)$ stellar contributions. Two features are particularly interestingly: (1) the C IV emission is surprisingly strong and (2) the He II emission is both strong and narrow, appearing mostly nebular in origin. The $\pm 1 \sigma$ error spectrum (shaded grey) is shown for comparison. Residuals to the fit are plotted below the corresponding spectral windows on a scale of -1 to 1 .

nebular emission $\left(\sim 280 \mathrm{~km} \mathrm{~s}^{-1}\right)$.

In the case of $\mathrm{C}$ IV, which is typically an interstellar medium (ISM) absorption feature or a P-Cygni profile from the stellar winds of massive stars, it is clearly seen in SL2S0217 as an emission doublet. Because the C IV doublet, like $\mathrm{Ly} \alpha$, can also be resonantly scattered, its profile widths were not constrained to the pure nebular line widths. The resulting best fit had an observed-frame Gaussian FWHM $6.0 \AA$ or about $410 \mathrm{~km} \mathrm{~s}^{-1}$. Compared to the nearby profiles of the $\mathrm{O}$ III] nebular lines $\left(285 \mathrm{~km} \mathrm{~s}^{-1}\right)$ this $45 \%$ increase in profile width indicates that $\mathrm{C}$ IV is likely dominated by nebular emission, but is broadened by resonant scattering. Additionally, strong emission may be masking an absorption component, and, therefore, we report the C IV emission line strengths as lower limits.

\section{3. $L y \alpha$}

Ly $\alpha$ emission is predominantly produced by recombination in the ionized gas surrounding star-formation, but

${ }^{4}$ IRAF is distributed by the National Optical Astronomical Observatories.

${ }^{5}$ For the current spectrum, the emission line shape is largely determined by the instrument resolution, which is wavelength dependent, and so the FWHM changes slightly across the spectrum $(\sim 1.5-1.7 \AA$ in the rest frame).

${ }^{6} \mathrm{~B} 12$ presented further evidence for minimal dust. They determined the extinction of the arc continuum to be $A_{V}=0.09$ from their 2D model fit to the observed 3D-HST grism spectrum using a Calzetti et al. (2000) reddening law. Assuming $R_{V}=4.05$, this corresponds to a very small reddening value of $E(B-V)=0.022$, and so was concluded to be negligible. 
TABLE 5

NEBULAR EMission Lines

\begin{tabular}{|c|c|c|c|c|c|}
\hline Ion & $\lambda_{\text {lab }}(\AA)^{a}$ & $F_{\lambda}^{b}$ & $W(\AA)^{c}$ & $I_{\lambda}^{b}$ & $I_{\lambda} / I_{\lambda 1909}$ \\
\hline $\operatorname{Ly} \alpha_{b}$ & 1215.67 & $58.7 \pm 0.6$ & 66.0 & $71.3 \pm 1.2$ & 8.94 \\
\hline $\operatorname{Ly} \alpha_{r}$ & 1215.67 & $39.0 \pm 0.4$ & 43.2 & $47.3 \pm 0.8$ & 5.94 \\
\hline $\operatorname{Ly} \alpha_{t o t}$ & 1215.67 & $100.8 \pm 1.0$ & 113.0 & $122.4 \pm 2.1$ & 15.3 \\
\hline \multirow[t]{2}{*}{ C IV } & 1548.19 & $5.54 \pm 0.07$ & 3.1 & $6.40 \pm 0.12$ & 0.80 \\
\hline & 1550.77 & $4.47 \pm 0.06$ & 2.5 & $5.17 \pm 0.10$ & 0.65 \\
\hline He II & 1640.42 & $4.62 \pm 0.07$ & 2.8 & $5.32 \pm 0.10$ & 0.67 \\
\hline He $\mathrm{II}_{n}$ & 1640.42 & $3.98 \pm 0.06$ & 2.4 & $4.58 \pm 0.09$ & 0.57 \\
\hline $\mathrm{He} \mathrm{II}_{w}$ & 1640.42 & $1.33 \pm 0.05$ & 0.8 & $1.53 \pm 0.07$ & 0.19 \\
\hline \multirow[t]{2}{*}{ O III] } & 1660.81 & $3.50 \pm 0.06$ & 2.1 & $4.03 \pm 0.09$ & 0.51 \\
\hline & 1666.15 & $7.60 \pm 0.09$ & 4.5 & $8.74 \pm 0.15$ & 1.10 \\
\hline $\mathrm{N}_{\text {IIII }}{ }^{d}$ & 1749 & $0.52 \pm 0.10$ & 0.4 & $0.60 \pm 0.12$ & 0.08 \\
\hline \multirow{2}{*}{ Si III] } & 1882.71 & $3.17 \pm 0.06$ & 2.1 & $3.66 \pm 0.08$ & 0.46 \\
\hline & 1892.03 & $2.35 \pm 0.05$ & 1.6 & $2.72 \pm 0.07$ & 0.34 \\
\hline [C III] & 1906.68 & $10.4 \pm 0.12$ & 7.0 & $12.1 \pm 0.19$ & 1.51 \\
\hline $\mathrm{C} \mathrm{III}]$ & 1908.73 & $6.90 \pm 0.08$ & 4.7 & $7.98 \pm 0.13$ & 1.00 \\
\hline [O III] & 2321.66 & $0.64 \pm 0.03$ & 0.6 & $0.74 \pm 0.08$ & 0.09 \\
\hline \multirow[t]{3}{*}{$\mathrm{C}$ II] } & 2325.40 & $0.36 \pm 0.03$ & 0.4 & $0.42 \pm 0.08$ & 0.05 \\
\hline & 2326.93 & $1.11 \pm 0.03$ & 0.9 & $1.29 \pm 0.09$ & 0.16 \\
\hline & 2328.12 & $0.27 \pm 0.03$ & 0.4 & $0.32 \pm 0.08$ & 0.04 \\
\hline$|\mathrm{Ne} \mathrm{III}|$ & 3870.16 & $30 \pm 4$ & $\cdots$ & $32.6 \pm 4.4$ & $\cdots$ \\
\hline & 4101.00 & $19 \pm 2$ & $\cdots$ & $21.1 \pm 2.2$ & $\ldots$ \\
\hline $\mathrm{H} \gamma^{e}$ & 4341.69 & $35 \pm 2$ & $\ldots$ & $37.8 \pm 2.2$ & $\ldots$ \\
\hline$[\mathrm{O} \text { III }]^{e}$ & 4364.44 & $5 \pm 3$ & $\ldots$ & $5.3 \pm 3.2$ & $\ldots$ \\
\hline & 4862.69 & $74 \pm 2$ & 517 & $78.9 \pm 2.3$ & $\ldots$ \\
\hline \multirow{2}{*}{ [O III] } & 4960.30 & $79 \pm 2$ & & $84.6 \pm 3.3$ & $\cdots$ \\
\hline & 5008.24 & $230 \pm 2$ & $2095^{f}$ & $244.2 \pm 4.1$ & $\ldots$ \\
\hline
\end{tabular}

Note. - Rest-Frame UV emission lines from this work and optical emission lines from the 3D-HST spectrum of B12. Emission features were fit with Gaussian profiles, where nearby lines were constrained to the same FWHMs and a single wavelength offset. The exceptions to this rule are the Ly $\alpha, \mathrm{C}$ IV, and $\mathrm{He}$ II profile fits, which have additional contributions from resonant scattering (Ly $\alpha$ and $\mathrm{C} \mathrm{IV}$ ) and stars (C IV and He II). The two fits listed for $\operatorname{Ly} \alpha_{b}$ and $\operatorname{Ly} \alpha_{r}$ correspond to blue and red Gaussian components, whereas Ly $\alpha_{t o t}$ was determined by integrating the profile as a whole. For He II, a single Gaussian fit is reported, as well as a twocomponent fit with narrow and (potential) wide parts. Following B12, who found negligible reddening in SL2S0217, we simply corrected for the galactic extinction $(E(B-V)=0.0194$; Schlafly \& Finkbeiner 2011). Line fluxes (observed-frame) and equivalent widths (rest-frame) are listed in Columns 3 and 4 , followed by the extinction corrected intensities in Column 5.

${ }^{a}$ Vacuum wavelengths.

${ }^{b}$ Units are $10^{-17} \mathrm{erg} \mathrm{s}^{-1} \mathrm{~cm}^{-2}$, uncorrected for lens magnification.

${ }^{c}$ Equivalent widths are measured in the rest-frame.

${ }^{d} \mathrm{~N}$ III] is the blend of $\mathrm{N}$ III] $\lambda \lambda 1748,1749$.

${ }^{e}$ Lines were completely blended in the $3 \mathrm{D}-H S T$ grism spectrum.

${ }^{f}$ Total EW of the blended [O III] $\lambda \lambda 4959,5007$ doublet.

also by collisionally excited neutral $\mathrm{H}$ gas (cooling radiation; Dijkstra 2014). However, the emission feature is increasingly obscured and scattered with increasing Hi column density and dust attenuation. Therefore, the shape of the Ly $\alpha$ profile depends on the kinematics and distribution of the outflowing gas and dust in star-forming galaxies (e.g., Verhamme et al. 2006; Steidel et al. 2010; Kornei et al. 2010).

The Ly $\alpha$ velocity profile from the LRIS spectrum of SL2S0217 is shown in Figure 6, where we observe strong, double-peaked emission with a dominant blue peak and nearly systemic central velocity. The total, integrated Ly $\alpha$ emission has a large equivalent width of $W_{L y \alpha}=113$ $\AA$, consistent with values for other young, metal-poor galaxies (e.g., Cowie et al. 2011, Trainor et al. 2016). Fitting the emission with two independent Gaussian profiles, we measure the relative blue to red flux to be $\sim 1.5$. The peak separation of this profile is $\Delta_{\text {peaks }}=371 \mathrm{~km}$ $\mathrm{s}^{-1}$. From Figure 6 we can see that this model fits the profile fairly well, however, some of the flux at the peaks is missed, and a residual blue tail extends to $\sim-1300$ $\mathrm{km} \mathrm{s}^{-1}$.

Multi-peaked Ly $\alpha$ emission (multiplicity) is commonly associated with star-forming galaxies. In fact, recent studies have found multiplicity rates of $30-50 \%$ amongst large samples of $z \sim 2-3$ star-forming galaxies (e.g., Kulas et al. 2012) and Ly $\alpha$ emitters (LAEs; e.g. Yamada et al. 2012; Trainor et al. 2015). Targets with Ly $\alpha$ multiplicity are typically grouped according to whether they are blue- or red-peak dominant. While targets with red dominant peaks are much more common than blue dominant peaks, Trainor et al. (2015) find that $26 \%$ of their 129 multi-peak LAEs are blue dominant, with an average peak separation of $\left\langle\Delta v_{\text {peaks }}\right\rangle=660 \pm 300 \mathrm{~km} \mathrm{~s}^{-1}$. Additionally, for blue dominant profiles in star-forming galaxies, Kulas et al. (2012) found an average peak separation of $\left\langle\Delta v_{\text {peaks }}\right\rangle \sim 800 \mathrm{~km} \mathrm{~s}^{-1}$, where the blueshifted peak is located at $v_{\text {peak }} \sim-200 \mathrm{~km} \mathrm{~s}^{-1}$. Note, however, that these results are based on low resolution spectra $\left(\Delta v \sim 200-600 \mathrm{~km} \mathrm{~s}^{-1}\right)$, which are more sensitive 


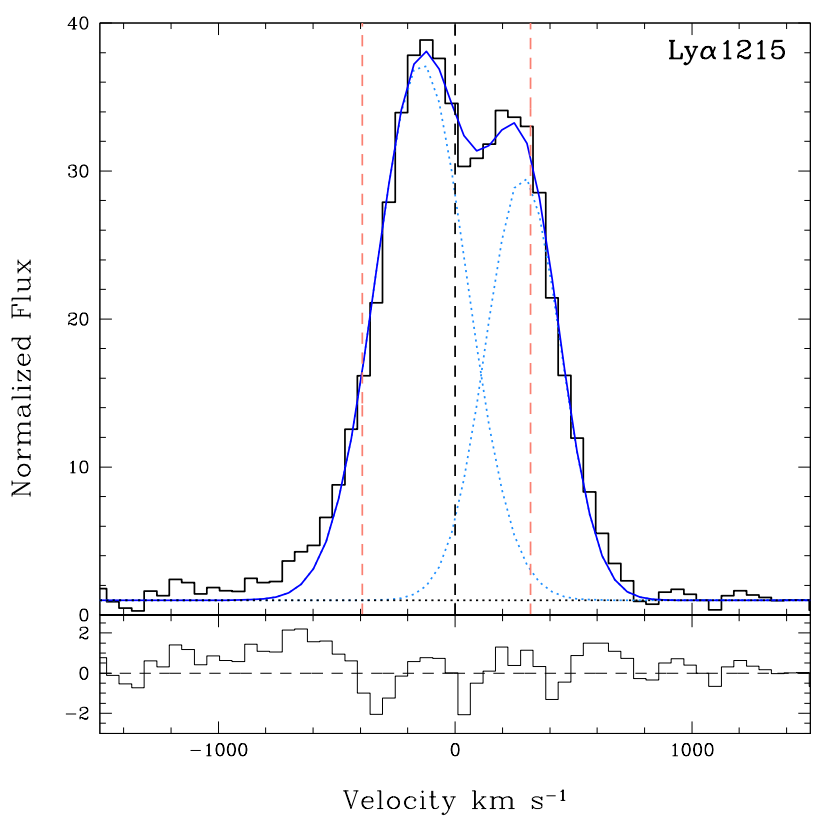

FIG. 6.- Velocity profile of the double-peaked Ly $\alpha$ emission in SL2S0217. The overall profile is reasonably well-fit using two Gaussians (blue lines), but a residual blue tail remains (shown in the bottom panel), indicating a more complex fit is needed to fully describe the kinematic properties of the Ly $\alpha$-emitting gas. Interestingly, the blue component is stronger than the red, and has a slightly larger velocity width. The Ly $\alpha$ peak separation is relatively small, shown here to be narrower than the velocity range of the average absorption feature (red dashed lines), with a nearly systemic central velocity.

to larger peak separations, and so may be biased to a higher average value. Still, the peak separation we observe for SL2S0217 $\left(\Delta v_{\text {peaks }} \sim 371 \mathrm{~km} \mathrm{~s}^{-1}\right)$ is small relative to these samples of star-forming galaxies at similar redshifts.

The simplest explanation for a double-peaked Ly $\alpha$ emission profile is scattering by a static, homogeneous, spherical or shell gas cloud. Given such a medium, the Ly $\alpha$ peak separation is set by the total Hi column density. For the peak separation and doppler parameter (thermal velocity dispersion) of $b=\sqrt{2 k_{B} T / m_{\mathrm{H}}}=16.3 \mathrm{~km} \mathrm{~s}^{-1}$ (for $T=16100 \mathrm{~K}$ ) measured in SL2S0217, the radiative transfer models of Verhamme et al. (2015) predict a column density of neutral gas of $N_{\mathrm{HI}} \lesssim 10^{20} \mathrm{~cm}^{-2}$. Observationally, the trend in $\mathrm{Ly} \alpha$ peak separation with $\mathrm{HI}$ column density found for $12 \operatorname{Ly} \alpha$ emitters at $z \sim 2$ by Hashimoto et al. (2015) also suggests $N_{\mathrm{HI}} \approx 10^{20} \mathrm{~cm}^{-2}$. However, static models produce red and blue emission components of equal strengths and widths, and so a more complex picture is needed to explain the larger intensity and wing of the blue Ly $\alpha$ peak in SL2S0217.

Additional explanations of the Ly $\alpha$ emission profile exist, including fluorescence, where ionizing photons that escape from galaxies can produce double-peaked fluorescent Ly $\alpha$ emission in cold clouds (e.g., Mas-Ribas \& Dijkstra 2016). Like the Ly $\alpha$ profile of SL2S0217, Huorescence tends to produce a small peak separation (e.g., Gould \& Weinberg 1996). Alternatively, Vanzella et al. (2017) used MUSE integral field spectroscopy of two extended Ly $\alpha$ systems to measure spatial-dependent emission and varying sub-structures, suggesting radiative transfer through clumpy/multiphase media as the source of blue-dominant Ly $\alpha$ emission (e.g., Gronke et al. 2016). Unfortunately, our observations of SL2S0217 lack sufficient resolution to assess the spatial extent of or variations in emission, and are further complicated by lensing.

Even in a simple static model, viewing Ly $\alpha$ emission from different inclinations / orientations affects the relative blue and red Ly $\alpha$ emission strengths (Behrens \& Braun 2014), especially in a differentially lensed system such as SL2S0217. Alternatively, in radiation transfer models, blue- and red-peak dominant Ly $\alpha$ morphologies are associated with inflowing and outflowing gas respectively (e.g., Dijkstra et al. 2006, Verhamme et al.|2006). In the case of infalling gas towards a central source, the red side of a double-peaked profile is depressed as the redshifted Ly $\alpha$ photons within the galaxy see higher optical depth due to the line-of-sight infalling gas, resulting in dominant blue-peak Ly $\alpha$ emission (see, e.g., Yang et al. 2012). The Ly $\alpha$ profile of SL2S0217 is consistent with this infalling halo model, which predicts a peak separation of $\left\langle\Delta v_{\text {peaks }}\right\rangle \sim 400 \mathrm{~km} \mathrm{~s}^{-1}$ (Verhamme et al.|2006 see their Figure 5). However, such a model does not eliminate the possibility of outflows, as we could have a restricted sight line along which the gas is static or cold pristine gas is flowing into the galaxy, and such that outflows are not visible to our viewing angle.

If we are in fact observing the effects of gas inflowing towards the star-forming regions in SL2S0217, then we may be witnessing an early episode of star formation during which we might have expected relatively low $N_{\mathrm{HI}}$ and negligible dust attenuation (inferred from the Balmer decrement of the grism spectrum) to favor a leakage of ionizing radiation. The fraction of escaping Ly $\alpha$ can be estimated by comparing the intrinsic and observed Ly $\alpha$ luminosities. We determine the intrinsic Ly $\alpha$ luminosity by multiplying the theoretical $\mathrm{Ly} \alpha / \mathrm{H} \beta$ ratio of 23.3 (assuming $T_{e}=1.5 \times 10^{4} \mathrm{~K}$ and $n_{e}=10^{2} \mathrm{~cm}^{-3}$; Osterbrock 1989) with the $\mathrm{H} \beta$ intensity (only corrected for galactic extinction; see Section 5.2). Note, however, that the theoretical $\mathrm{Ly} \alpha / \mathrm{H} \beta$ ratio is density sensitive, and so we also consider the $n_{e}=10^{3} \mathrm{~cm}^{-3}$ case in which $\mathrm{Ly} \alpha / \mathrm{H} \beta=25.7$. Finally, we use the total magnification $\left(\mu_{\text {tot }}=17.3\right)$ to correct the observed $\mathrm{H} \beta$ intensity and the effective mean magnification $\left(\mu_{e f f}=19\right)$ to correct the observed Ly $\alpha$ intensity. Interestingly, the Ly $\alpha$ escape fraction, $f_{e s c}^{L y \alpha}$, is $L_{L y \alpha}^{o b s} / L_{L y \alpha}^{i n t}=0.061(0.055)$ for $n_{e}=10^{2}\left(10^{3}\right) \mathrm{cm}^{-3}$, or only $6 \%$ of Ly $\alpha$ emission escapes along the line of sight.

For a sample of local Ly continuum (LyC) emitters, Verhamme et al. (2017) found that both Ly $\alpha$ and LyC escape fractions increased with increasing Ly $\alpha$ EWs, while Ly $\alpha$ peak separations decreased. Along these lines, the small Ly $\alpha$ peak separation $\left(371 \mathrm{~km} \mathrm{~s}^{-1}\right)$ and large EW in SL2S0217 (113 $\AA$ ) argues for significant Ly $\alpha$ leakage ( 40 - 60\%; Verhamme et al. 2017), discordant with the observed estimated escape fraction. Note, however, that the Ly $\alpha$ escape fraction may be underestimated due to slit losses. A preliminary analysis of the continuumsubtracted HST WFC3 F343N image suggests significant Ly $\alpha$ slit losses of roughly $30-40 \%$. Correcting the Ly $\alpha$ flux for this factor increases the Ly $\alpha$ escape fraction to $10 \%$, still surprisingly small given the large observed Ly $\alpha$ 
EW. A complete analysis will be discussed in a future paper (Erb et al. 2018, in prep.).

While SL2S0217 is a clear outlier to the trends found for the sample of LyC emitters in Verhamme et al. (2017), Jaskot et al. (2017) recently reported an extreme Green Pea galaxy, J1608, with properties similar to SL2S0217. In particular, these authors found J1608 to be a very high ionization (from $[\mathrm{O} \mathrm{III}] /[\mathrm{O} \mathrm{II}]$ ) galaxy, with no evidence of outflows, and measured a Ly $\alpha$ escape fraction of 0.16 , despite its strong Ly $\alpha$ EW of $163 \AA$ and narrow peak separation of $\Delta_{\text {peaks }}=214 \mathrm{~km} \mathrm{~s}^{-1}$. They suggest that multiple mechanisms for LyC escape exist, where such extreme targets may have suppressed superwinds, with radiation dominated feedback driving Ly $\alpha$ escape, and likely escaping LyC emission. Similarly, despite the low Ly $\alpha$ escape fraction measured for SL2S0217, it has several characteristics, namely strong [O III $] /[\mathrm{O}$ II $]$, large Ly $\alpha \mathrm{EW}$, and small Ly $\alpha$ peak separation, that are indicative of LyC leakage (Henry et al. 2015, Izotov et al. 2016 Verhamme et al. 2017). SL2S0217 is, therefore, a unique template to probe the conditions of extreme galaxies that may have played a critical role in the reionization of the Universe.

\subsection{Interstellar Absorption Lines}

Outflowing gas, if present, can be directly probed by examining the interstellar absorption line profiles. Given sufficient spectral resolution and signal-to-noise in these features, a map of the covering fraction of the absorbing gas as a function of velocity can be inferred for both highand low-ionization states. To better understand the gas kinematics in SL2S0217, the UV spectrum was normalized using the Rix et al. (2004) continuum windows as a guide, with further continuum designated by eye.

The normalized profiles of the significant absorption line detections are depicted in Figure 7, arranged by ion. Many of the strong, low-ionization absorption lines that are characteristic of $z \sim 2-3$ galaxies (e.g., Pettini et al. 2002b. Shapley et al. 2003) are also present in the SL2S0217 spectrum, but with $(\sim 3 \times)$ narrower velocity ranges. We can place constraints on the absorbing gas by measuring the properties of these profiles for various transitions. Using a bootstrap Monte Carlo simulation in which the $1 \sigma$ uncertainty was used to perturb a given absorption profile and generate 1000 artificial spectra, we measured the flux-weighted centroid, velocity range, and integrated equivalent width for each line. These values, along with the oscillator strengths for each line (Morton 2003), are given in Table 6. The integrated profiles are depicted by the filled absorption features in Figure 7

Measurements of two different lines from the same ion can be used to assess the optical depth quantitatively. On the linear part of the curve of growth, $W \propto N f \lambda^{2}$, where $W$ is the equivalent width, $N$ is the column density, $f$ is the oscillator strength, and $\lambda$ is the wavelength of a given transition. Then, the theoretical ratio of the Si II lines is $W_{\lambda 1527} / W_{\lambda 1808}=45.2$, such that Si II $\lambda 1527$ is expected to be about 45 times stronger than Si II $\lambda 1808$ in the optically thin case. Instead, the observed ratio of $W_{\lambda 1527} / W_{\lambda 1808}=1.136 \AA / 0.271 \AA=4.2$ indicates line saturation and optically thick gas. Similar results are found for the low-ionization Fe II $\lambda \lambda 1608,1611$ lines (theoretical $W_{\lambda 1608} / W_{\lambda 1611}=41.7$ versus 2.7 observed), suggesting the Fe II $\lambda 1608$ line is strongly saturated. Deviations are also found for the high-ionization Si IV $\lambda \lambda 1394,1403$ lines, but to a smaller degree, as the observed ratio is about half of the theoretical ratio. Therefore, all but perhaps the weakest absorption lines in the UV SL2S0217 spectrum are saturated to varying degrees. In this case, the equivalent width is dependent on both the velocity range and covering fraction of the absorbing gas, but high resolution is needed to model the covering fraction as a function of velocity. Given the low resolution of the UV SL2S0217 spectrum (FWHM $280 \mathrm{~km}$ $\mathrm{s}^{-1}$ at $1500 \AA$ ), a more detailed kinematic analysis of individual lines is unfeasible at this time.

\subsection{Average Absorption Profiles}

Differences between the average low- and highionization line profiles can inform gas properties. We determined error-weighted average velocity profiles from the $(i)$ low-ionization, (ii) high-ionization, and (iii) combined absorption features for comparison. Given that the near-UV spectrum is significantly more affected by night sky contamination, and the Fe II profiles may be affected by emission filling (see $\S 5.6$ ), absorption features from the red side were not used in the average profile. From the blue spectrum, the S II + Si II $\lambda 1260$ feature was excluded from the average due to its blended nature. Far-UV regions affected by night-sky contamination were also omitted. The combined average profile, therefore, incorporated the appropriate portions of $\mathrm{O}_{1}$ $\lambda 1302$, Si II $\lambda 1304$, Si IV $\lambda 1394$, Si IV $\lambda 1403$, Si II $\lambda 1527$, Fe II $\lambda 1608$, Fe II $\lambda 1611$, and $\mathrm{Al}$ II $\lambda 1670$, indicated by the black profiles in Figure 7

The resulting profiles are nearly identical. In Figure 7 the combined average profile (blue) is overplotted on the individual absorption features, where the portions of each line used in the average are designated by a solid black line. Apparently the low- and high-ionization gas has similar kinematic properties, as all the observed species seem to be nominally consistent with the average profile. Using a bootstrap Monte Carlo simulation in which the $1 \sigma$ uncertainty was used to perturb the average profile and generate 1000 artificial spectra, we measured a flux-weighted centroid of $-7.8 \mathrm{~km} \mathrm{~s}^{-1}$, with limits of $[-392,+319] \mathrm{km} \mathrm{s}^{-1}$ (designated by red vertical lines in Figure 7), and an equivalent width of $0.80 \AA$. Despite our reticence to interpret the gas kinematics (due to line saturation and low-resolution), the average absorption profile of SL2S0217 is clearly uncharacteristic in terms of both velocity centroid and range relative to interstellar absorption observed in other $z \sim 2$ galaxies (e.g., Pettini et al. 2000, Quider et al. 2010, Erb et al. 2010). Rather, the average profile is characterized by nearly symmetric, narrow absorption around the systemic velocity, a signature of little-to-no outflowing gas.

In Figure 8 we compare the average velocity profiles of the low- and high-ionization absorption lines to the $\mathrm{O}$ III] emission features present in the UV spectrum of SL2S0217. Evidently, the absorption velocity profiles are remarkably similar to those of the emission lines, whose widths represent the instrumental profile, providing a vivid demonstration of the lack of outflows in SL2S0217.

\subsection{Fine Structure and Resonant Emission Lines}




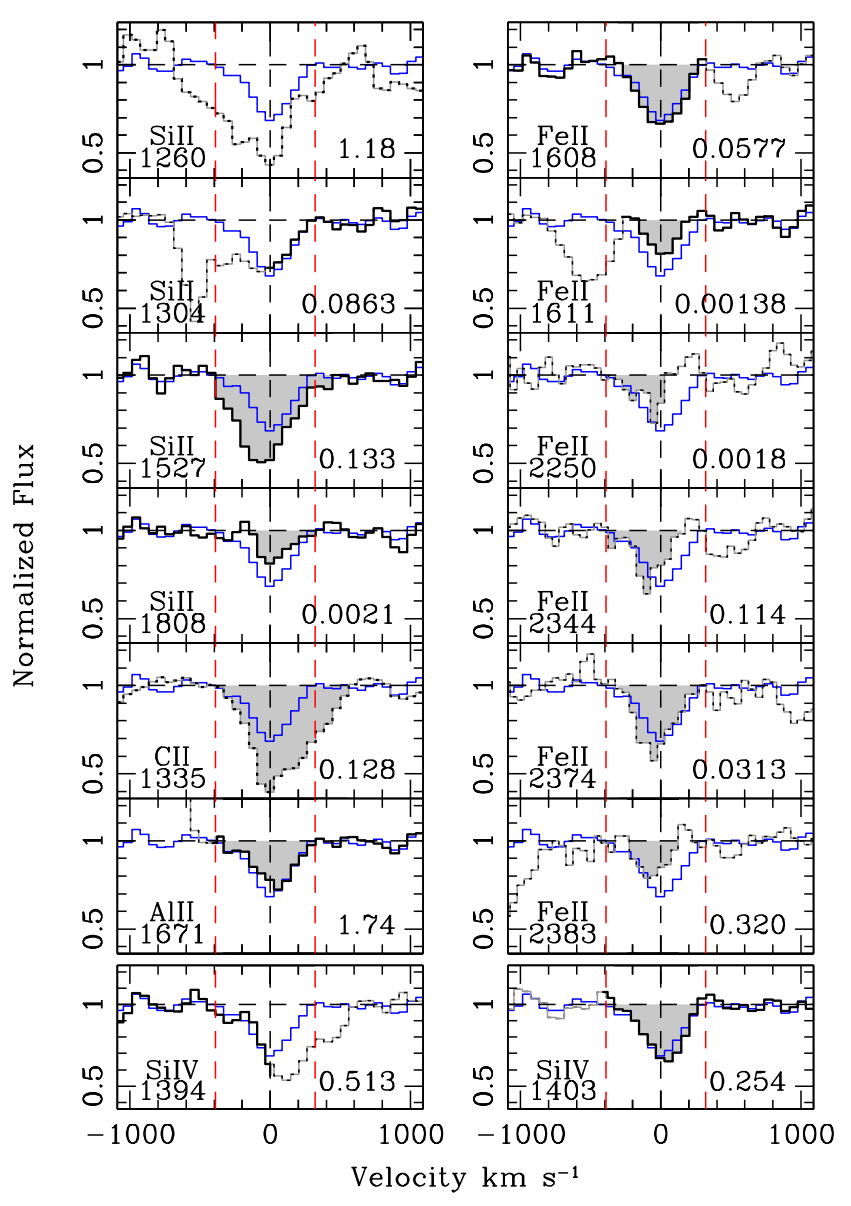

FIG. 7.- Absorption features of both low and high ionization species in SL2S0217. The blue line represents the average absorption profile, composed of the portions of the spectra with thick, solid black lines. The average profile is centered close to $0 \mathrm{~km} / \mathrm{s}$ and is nearly symmetric, with velocity limits of $[-392,+319] \mathrm{km} / \mathrm{s}$ (red, dashed vertical lines). In comparison to the $f$-values (listed in the lower right-hand corner for each line), the strongest lines must be saturated.

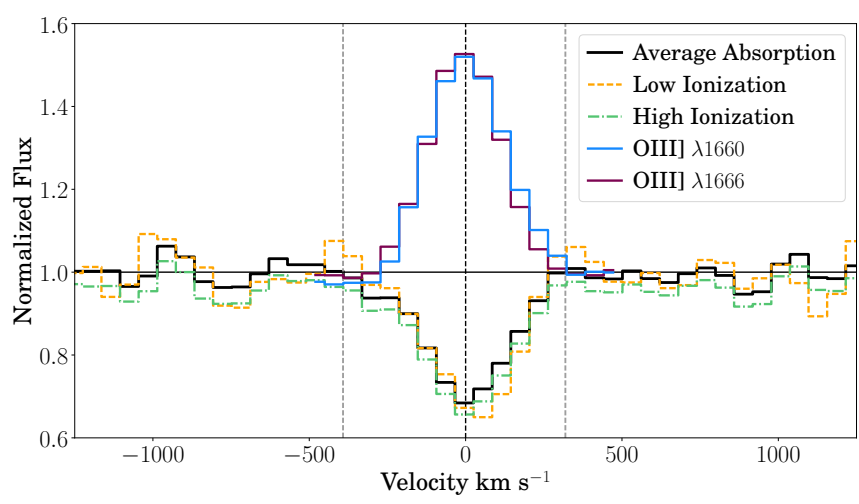

FiG. 8. - Comparison of the emission and absorption velocity profiles in SL2S0217. The strengths of the emission lines are arbitrarily scaled for visualization. An average profile was determined for the low-ionization species by combining the individual profiles (see Figure 7), whereas Si IV $\lambda 1403$ alone was used to represent the high-ionization profile. Remarkably, the emission and absorption profiles are well-matched in terms of line width and velocity centroid.
Fine structure lines, such as Si II* $\lambda \lambda \lambda 1265,1309,1533$, $\mathrm{C}$ II $^{*} \lambda 1335$, and $\mathrm{Fe} \mathrm{II}^{*} \lambda \lambda 2365,2396$, are clearly visible and redshifted in other $z \sim 2$ emission-line galaxies such as BX418 (emission; Erb et al. 2010) and cB58 (absorption; Pettini et al. 2000), but are not detected in the UV spectra of local starbursts (e.g., Schwartz et al. 2006; Leitherer et al. 2011). Additionally, previous studies have found correlations between strong Fe II* features and/or Mg II $\lambda \lambda 2796,2803$ resonant emission and galaxies with extreme properties similar to SL2S0217, namely, high sSFRs, low stellar masses, blue UV slopes, and large Ly $\alpha$ equivalent widths (e.g., Erb et al. 2012; Jones et al. 2012 Kornei et al. 2013). Based on these properties alone, we might have expected to see Fe fine structure or $\mathrm{Mg}$ resonant emission in our spectrum of SL2S0217, however, we find no evidence of these features. On the other hand, from a sample of $184 z \sim 1$ star-forming galaxies, $\mathrm{Du}$ et al. (2017) find that Fe II* strength does not vary significantly with stellar mass or color. While the significance of these trends is still a matter of debate, the fact that we do not significantly detect any of the Fe II* or $\mathrm{Mg}$ II features is interesting.

Previous attempts to reproduce the fine structure emission lines using CLOUDY photoionization models have been unsuccessful, suggesting that these lines are not likely produced by nebular photoionization (Shapley et al. 2003: Erb et al. 2010). Erb et al. (2010) measure a consistent redshift of the fine structure lines in the $z=2.3$ galaxy Q2343-BX418 by $\sim 200 \mathrm{~km} \mathrm{~s}^{-1}$ and suggest an origin in the outflowing gas, potentially through resonant scattering, but caution that this theory is incomplete as it does not explain the narrowness of the observed lines. Rubin et al. (2011) proposed that Fe II* emission, observed at or near the systemic velocity, arises from photon scattering in outflowing gas, and so may trace the spatial extent of the outflows. This scenario is supported by the nearly systemic mean velocity centroid for the sample of $96 z \sim 1-2$ star-forming galaxies analyzed by Erb et al. $\left(\Delta v_{2626}=-20 \mathrm{~km} \mathrm{~s}^{-1} ; 2012\right)$.

The absence of Fe II fine-structure features in SL2S0217 could then be due, in part, to the lack of outflowing gas observed along our line of sight (see discussion of the velocity structure of the absorption lines in Section 5.4). Nevertheless, if scattering in outflows is in fact the production mechanism responsible for $\mathrm{Fe} \mathrm{II}^{*}$ emission, then extended emission in transverse outflows, and subsequent slit loses, are possible. Note, however, that these weak features may simply not be detectable given the low resolution and signal-to-noise of the LRIS spectrum.

Galactic outflow models have shown that the overall profiles of the Fe II and $\mathrm{Mg}$ II lines can be significantly altered by the effects of photon scattering and re-emitted photons (Scarlata \& Panagia 2015). Emission filling, where the re-scattered emission "fills in" a portion of the absorption profile, may then be responsible for the bluer velocity centroids and reduced equivalent widths of many of the near UV Fe II profiles depicted in Figure 7 and listed in Table 6 (see, also, Erb et al. 2012). The effects of re-scattered emission may be even greater for Fe II $\lambda 2383$, whose only allowed transition after absorption is back to the ground state.

$\mathrm{Mg}$ II absorption profiles may be filled by both resonance re-emission and nebular emission (e.g., Henry et al. 
TABLE 6

Interstellar ABsorption Lines

\begin{tabular}{cccccc}
\hline \hline Ion & $\begin{array}{c}\lambda_{\text {lab }} \\
(\AA)\end{array}$ & $f$-value & $\begin{array}{c}v^{a} \\
\left(\mathrm{~km} \mathrm{~s}^{-1}\right)\end{array}$ & $\begin{array}{c}\Delta v^{b} \\
\left(\mathrm{~km} \mathrm{~s}^{-1}\right)\end{array}$ & $\begin{array}{c}W_{0}{ }^{c} \\
(\AA)\end{array}$ \\
\hline C II & $1334.5323^{d}$ & 0.128 & 93 & $-350:+525$ & $1.279 \pm 0.119$ \\
Si IV & 1402.7729 & 0.254 & 14 & $-314+282$ & $0.511 \pm 0.063$ \\
Si II & 1526.7070 & 0.133 & -37 & $-432:+445$ & $1.136 \pm 0.102$ \\
Fe II & 1608.4511 & 0.0577 & 4 & $-332:+292$ & $0.643 \pm 0.072$ \\
Fe II & 1611.2005 & 0.00138 & 6 & $-197:+201$ & $0.237 \pm 0.040$ \\
Al II & 1670.7886 & 1.74 & 19 & $-317:+342$ & $0.489 \pm 0.088$ \\
Si II & 1808.0129 & 0.0021 & 39 & $-154:+310$ & $0.271 \pm 0.058$ \\
Fe II & 2249.8768 & 0.0018 & -95 & $-299:+61$ & $0.315 \pm 0.079$ \\
Fe II & 2344.2189 & 0.114 & -83 & $-323:+149$ & $0.567 \pm 0.074$ \\
Fe II & 2374.4612 & 0.0313 & -27 & $-303:+282$ & $0.927 \pm 0.107$ \\
Fe II & 2382.7652 & 0.320 & -63 & $-247:+124$ & $0.395 \pm 0.087$ \\
Ave. & & & -8 & $-392:+319$ & $0.805 \pm 0.050$ \\
\hline
\end{tabular}

Note. - Measurements of the cleanest absorption profiles in SL2S0217. Vacuum wavelengths and $f$-values are taken from Morton (2003). Columns 4 and 5 list the flux-weighted velocity centroids and velocity ranges over which the profiles were integrated in order to determined the equivalent widths given in Column 6. Characteristics of the error-weighted average absorption profile are given in the final row for comparison.

${ }^{a}$ Velocity relative to $z_{\text {sys }}=1.844$ (see Table 2.

${ }^{b}$ Velocity range used for equivalent width measurements relative to line center (Column 2) at $z_{\text {sys }}=1.844$.

${ }^{c}$ Rest-frame equivalent width.

${ }^{d}$ The unresolved blend of $\mathrm{C}_{\text {II }} \lambda 1334.5323+\mathrm{C}_{\text {II }}{ }^{*} \lambda 1335.6627$

TABLE 7

IntERstellar ABUNDANCES

\begin{tabular}{lccccc}
\hline \hline Ion & $\lambda(\AA)$ & $\log \left(N_{\mathrm{HI}} \mathrm{cm}^{-2}\right)$ & $\log (\mathrm{X} / \mathrm{H})$ & $\log (\mathrm{X} / \mathrm{H})_{\odot}^{b}$ & {$[\mathrm{X} / \mathrm{H}]^{c}$} \\
\hline H I & 1215 & $20.0^{a}$ & - & - & - \\
Fe II & 1611 & 15.874 & -4.126 & -4.50 & 0.374 \\
Si II & 1808 & 15.649 & -4.351 & -4.49 & 0.139 \\
\hline
\end{tabular}

Note. - Interstellar abundances estimated from the weakest absorption features, assuming the optically thin case.

${ }^{a}$ Value of $\log \left(N_{\mathrm{HI}} \mathrm{cm} \mathrm{s}^{-1}\right)$ predicted by homogeneous shell models with no expansion velocity, $b=10 \mathrm{~km} \mathrm{~s}^{-1}$, and $\Delta v=371 \mathrm{~km} \mathrm{~s}^{-1}$ Verhamme et al. 2015).

${ }^{b}$ Solar (meteoric) abundances from the compilation by Asplund et al. (2009).

${ }^{\mathrm{c}}[\mathrm{X} / \mathrm{H}]=\log (\mathrm{X} / \mathrm{H})-\log (\mathrm{X} / \mathrm{H}) \odot$.

2018). From their composite spectra, Du et al. (2017)

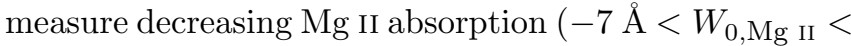
$-1 \AA$ ) with increasing $\mathrm{C}$ III] emission, attributing the effect to nebular emission filling. From the scatter in the LRIS spectrum, we estimate the upper limit of the Mg II features to be $W_{0, \mathrm{Mg} \text { II }} \sim 0.5 \AA$; only a small residual feature is possible, as expected for nebular and, possibly, significant re-scattered emission line filling in a target with little-to-no outflows.

\section{ABSORPTION LINE ABUNDANCES}

While most of the absorption lines in the UV spectrum of SL2S0217 are likely saturated, the weakest lines offer the best opportunity to constrain relative abundances. Using the integrated equivalent widths from Table 6, we estimated the column density for the weakest lines observed, assuming optically thin conditions (apparent optical depth method). From Spitzer (1978), the linear part of the curve of growth can be characterized as

$$
N=1.13 \times 10^{20}\left(\frac{W_{\lambda}}{\lambda^{2} f}\right)\left(\mathrm{cm}^{-2}\right),
$$

where $N$ is the column density, $\lambda$ is the transition wavelength, and $f$ is the oscillator strength (see Table 6). The resulting $N$ values are listed in Table 7 , however, if any of the lines are saturated, the column densities determined here should be considered lower limits.

Because the Ly $\alpha$ profile is seen purely in emission (see discussion in $\S 5.3$, we do not have a measure of the total column density of neutral hydrogen. As discussed in $\S 5.3$ the static, homogeneous radiative transfer shell models of Verhamme et al. (2015) predict a column density of neutral gas of $N_{\mathrm{HI}}=10^{20} \mathrm{~cm}^{-2}$ for the Ly $\alpha$ peak separation in SL2S0217. Adopting this value as indicative, we subsequently estimated the element abundances for Si II and Fe II, as reported in Table 7.

Since Si II and Fe II both have lines with very weak and similar oscillator strengths, assuming these lines are unsaturated, we can estimate the Si II/Fe II ratio (independent of $N_{\mathrm{HI}}$ ). Further, Si II and Fe II have similar ionization potentials and are the only ionization states that we observe in absorption for $\mathrm{Si}$ and $\mathrm{Fe}$, so the ionization correction factor will be small, and, to first order, can be ignored. Using our measured values for SL2S0217 in Table 7, we find $\log (\mathrm{Si} / \mathrm{Fe})=-0.225 \pm 0.118$, or $44-77 \%$ solar. However, Si is expected, both empirically and theoretically, to be overabundant relative to $\mathrm{Fe}$ in metalpoor dwarf galaxies (e.g., Tolstoy et al. 2009). This is because $\mathrm{Si}$ is predominantly an alpha element $(\alpha)$, produced on relatively short timescales during type II supernovae (SNe; massive stars) explosions, whereas Fe is synthesized by SNe Ia (intermediate binaries with mass transfer) and returned much later. Since SNe Ia have longer timescales than SNe II, Si and Fe trace different stellar populations and time scales. Enhanced $[\alpha / \mathrm{Fe}]$ occurs until SNe Ia begin to contribute to the chemical evolution $10^{8}-10^{9}$ years after the first episode of starformation.

Given the derived properties of SL2S0217, one may have expected a pronounced alpha-enhancement in a galaxy experiencing a young burst of star formation. Instead, the abnormal Si/Fe ratio measured for SL2S0217 could be evidence of an old stellar population that has had sufficient time to enrich the gas in Fe. Alternatively, the $[\alpha / \mathrm{Fe}]$-poor gas could be the result of larger $\mathrm{Si}$ than Fe depletion onto dust grains, although the authors of this work are not aware of any empirical evidence to support this scenario. The UV emission line ratios indicate that the Si depletion may be as great as 65\% in SL2S0217 (see $\S 7.2 .3$. Unfortunately, we cannot estimate the Fe depletion from emission lines with existing data. The Si abundance and dust depletion are further discussed in $\S 7.2 .3$.

\section{NEBULAR EMISSION LINE ABUNDANCES}

\subsection{Physical Parameters of the ISM}

Directly measuring abundances from nebular emission lines requires knowledge of the physical properties of the emitting gas. While detailed observations of nearby starforming regions reveal complex nebular structures (e.g., Pellegrini et al. 2012), simplified H II region models leverage a spherical geometry with three separate ionization volumes. The advantage of such models is that they account for the fact that the low-, intermediate-, and highionization zones are governed by different physical prop- 
erties, and therefore require reliable electron temperature and densities for each volume to determine accurate abundances. Although the SL2S0217 arc is evidently composed of multiple star-forming regions, they are not easily disentangled from the UV spectrum, and so we employ a single $\mathrm{H}$ II region model to estimate the nebular gas properties. The electron temperature and electron density of SL2S0217 were determined using the PYNEB package in PYTHON (Luridiana et al. 2012, 2015), assuming a five-level atom model (De Robertis et al. 1987).

\subsubsection{Electron Temperature}

Electron temperature is typically determined by observing a temperature-sensitive auroral-to-strong-line ratio. Historically, the [O III $] \lambda 4363 /[\mathrm{O}$ III $] \lambda 5007$ emission line ratio has been the ideal measure of electron temperature in the high-ionization zone of nebulae. However, the temperature-sensitive [O III] $\lambda 4363$ auroral line is not resolved in the $H S T$ grism spectrum.

Fortunately, the electron temperature can also be determined from the $\mathrm{O}$ III $] \lambda 1666 /[\mathrm{O}$ III $] \lambda 5007$ ratio, as is commonly done in high redshift targets where the intrinsically faint optical auroral line is often undetected. In the case of SL2S0217, this diagnostic combines spaceand ground-based observations, potentially introducing flux and aperture mismatching issues, and so this calculation must be done carefully. Given the excellent flux calibration of the HST grism spectrum and the match of the flux-corrected LRIS continuum to the HST ACS F606W AB magnitude, we used the $\mathrm{O}$ III $] \lambda 1666 /[\mathrm{O}$ III $] \lambda 5007$ ratio to measure $T_{e, \lambda 1666}=15,400 \pm 200 \mathrm{~K}$. Adopting this $T_{e, \lambda 1666}$ measurement for the high ionization zone electron temperature, the intermediate- and low-ionization zone temperatures $\left(T_{e}[\mathrm{~S} \mathrm{III}]\right.$ and $T_{e}[\mathrm{O} \mathrm{II}]$ respectively) were then determined from the theoretical temperature relationships of Garnett (1992). The temperatures used for each ionization zone are listed in Table 8.

Interestingly, Brammer et al. (2012) reported excess emission from the $\mathrm{H} \gamma+[\mathrm{O}$ III $] \lambda 4363$ blend in the grism spectrum, which can be attributed to [O $\mathrm{III}] \lambda 4363$ and used to constrain the optical electron temperature diagnostic. We determined the $\mathrm{H} \gamma$ flux contribution to the blend using the measured $\mathrm{H} \beta$ grism flux $\left(74 \pm 2 \times 10^{-17} \mathrm{erg} \mathrm{s}^{-1} \mathrm{~cm}^{-2}\right)$ and the Case-B $\mathrm{H} \gamma / \mathrm{H} \beta$ value from Hummer \& Storey (1987) assuming $T_{e}=$ $15,000 \mathrm{~K}$ and $n_{e}=100 \mathrm{~cm}^{-3}$ (conditions representative of SL2S0217; see Section 7.1.2 for $n_{e}$ ). Subtracting this $\mathrm{H} \gamma$ value from the blend $\left(40 \pm 3 \times 10^{-17} \mathrm{erg} \mathrm{s}^{-1}\right.$ $\mathrm{cm}^{-2}$; B12), the flux corresponding to [O III] $\lambda 4363$ is $F_{\lambda 4363}=4.9 \times 10^{-17} \mathrm{erg} \mathrm{s}^{-1} \mathrm{~cm}^{-2}$. We use the standard [O III] $\lambda 4363 /[\mathrm{O}$ III $] \lambda 5007$ temperature diagnostic to estimate the high-ionization zone electron temperature to be $T_{e, \lambda 4363}=15,700 \pm 3,200 \mathrm{~K}$, in agreement with the combined UV/optical temperature diagnostic. A higher signal-to-noise and resolution rest-frame optical spectrum would be useful to more securely measure [O III] $\lambda 4363$ and compare the two temperature diagnostics.

\subsubsection{Electron Density}

In nearby objects, the gas phase electron density is most commonly determined from the $[\mathrm{S}$ II $]$ and $\left[\begin{array}{ll}\mathrm{O} & \mathrm{II}\end{array}\right.$ optical collisionally-excited doublets. For SL2S0217, the
[O II] lines were not detected and the [S II] lines were outside the wavelength range covered by the $H S T$ grism spectrum. Instead, we observe two sets of densitysensitive UV emission-line doublets in our LRIS spectrum: Si III] $\lambda \lambda 1883,1892$ and C III] $\lambda \lambda 1907,1909$. We calculate densities of $4,500_{-1,400}^{+1,500} \mathrm{~cm}^{-3}$ and $300_{-300}^{+1,300}$ $\mathrm{cm}^{-3}$ for the Si III] and C III] ions respectively ${ }^{7}$. Since the relevant lines are all high $\mathrm{S} / \mathrm{N}$ and should result purely from nebular emission, we find no obvious explanation for this discrepancy.

The critical densities of $\mathrm{C}$ III] and Si III] are both of the order of $10^{4} \mathrm{~cm}^{-3}$, and so they are generally not useful density diagnostics for $n_{e}$ values below $\sim 10^{3} \mathrm{~cm}^{-3}$. This is evident in Figure 9, where we plot the emissivity ratios of common rest-frame $\mathrm{UV}$ ( $\mathrm{Si}$ III] and $\mathrm{C}$ III]) and optical ([O $\left[\begin{array}{ll}\mathrm{O}\end{array}\right]$ and $[\mathrm{S} \mathrm{II}]$ ) electron density diagnostic ratios, assuming a general electron temperature of $15,000 \mathrm{~K}$. When measured, the optical line ratios provide constraints below $n_{e} \sim 10^{3} \mathrm{~cm}^{-3}$, however, they probe a different (low-) ionization zone, and indicate significantly lower densities relative to the highly-ionized gas in high-redshift star-forming galaxies (e.g., Christensen et al. 2012; Bayliss et al. 2014, James et al. 2014). Traditionally, the high-ionization zone $(\sim 35-55 \mathrm{eV})$ is represented by $\mathrm{O}^{++}$, whereas the low-ionization zone $(\sim 15-35 \mathrm{eV})$ is represented by the $\mathrm{O}^{+}$or $\mathrm{N}^{+}$ions, with the intermediate-zone, based on $\mathrm{S}^{++}$, partially overlapping $(\sim 23-35 \mathrm{eV})$. By these definitions, the UV $\mathrm{Si}$ III] and $\mathrm{C}$ III density diagnostics span the low-tointermediate and intermediate-to-high ionization zones respectively. Perhaps not surprisingly, the mixed-zone electron densities measured for high-redshift targets, including SL2S0217, are significantly larger than the pure low-ionization zone densities typical of $\mathrm{H}$ II regions in nearby galaxies. Further, Sanders et al. (2016) find that $z \sim 2$ galaxies have mean electron densities that are an order of magnitude higher relative to local galaxies at fixed stellar mass.

While the insensitivity of the UV density diagnostics to low densities inhibits the density determination for SL2S0217, the C III] ratio is at least in agreement with the low-density regime, and so we assumed a standard value of $n_{e}=100 \mathrm{~cm}^{-3}$. Fortunately, the gas temperature and line emissivities are practically independent of the gas density in the low-density regime, and so this choice of density makes little difference. In fact, subsequently derived abundance ratios for both $n_{e}=100$ $\mathrm{cm}^{-3}$ (consistent with the $n_{e}$ from C III]) and $n_{e}=4,500$ $\mathrm{cm}^{-3}$ (from Si III]), given in Table 8, show differences that are significantly smaller than the respective uncertainties. However, detailed studies of these diagnostics must be prioritized to understand whether targets such as SL2S0217 have abnormally high electron densities/density inhomogeneities, the UV diagnostics probe a higher density regime than their optical counterparts, or some other effect is at play.

7 We note that a preliminary Si III] density determination using TEMDEN in IRAF produced unphysical results for SL2S0217. We subsequently found that the emissivity ratios for the Si III] density diagnostic approach an unexpected value of 3 using the default atomic data in IRAF. Alternatively, we find that the PyNeb package in Python uses the correct Si III] emissivities. 
TABLE 8

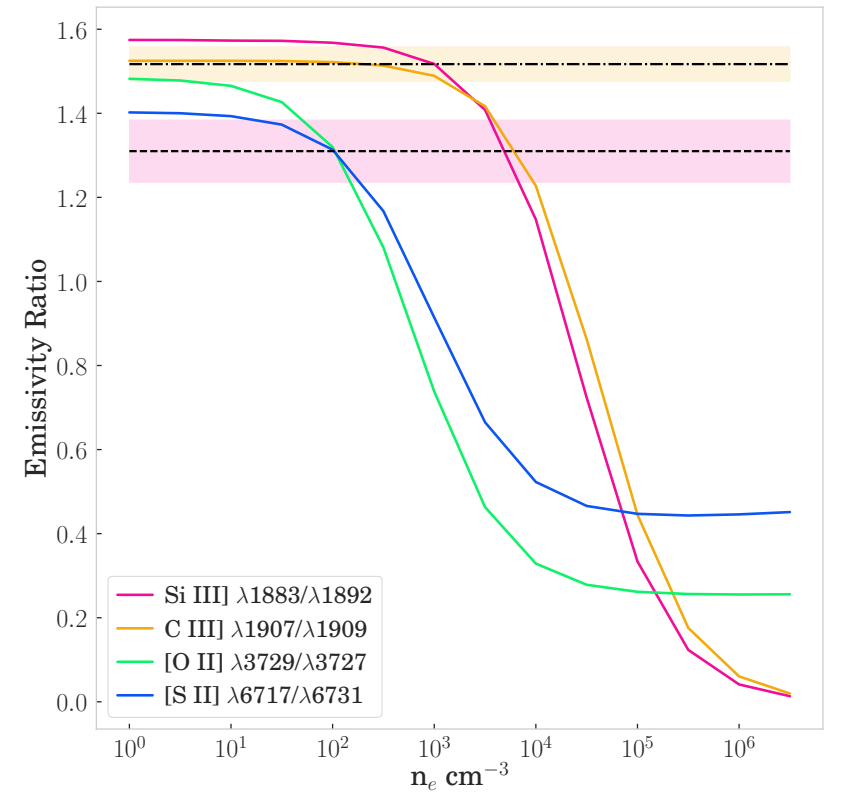

FIG. 9.- A comparison of common rest-frame UV (Si III] and $\mathrm{C}$ III] $)$ and optical ([O $\mathrm{OI}]$ and $[\mathrm{S} \mathrm{II}]$ ) electron density diagnostic ratios. Emissivities were calculated using the $\mathrm{PyNeb}$ package in Python, assuming an electron temperature of 15,000 K. The dashed (dashed-dotted) black line and pink (orange) box represent the Si III] (C III) ratio and uncertainty for SL2S0217. Unfortunately, neither $\mathrm{C}$ III] or Si III] are sensitive to low densities, so we simply assume a typical nebular density of $n_{e}=100 \mathrm{~cm}^{-3}$.

\subsection{Ionic And Total Abundances}

Due to the number of strong rest-frame UV emission lines observed in the spectrum of SL2S0217, we were able to determine relative $\mathrm{C}, \mathrm{O}, \mathrm{N}$, and $\mathrm{Si}$ abundances. Using the rest-frame optical grism spectrum, ionic abundances were also determined relative to hydrogen:

$$
\frac{N\left(X^{i}\right)}{N\left(H^{+}\right)}=\frac{I_{\lambda(i)}}{I_{H \beta}} \frac{j_{H \beta}}{j_{\lambda(i)}} .
$$

The emissivity coefficients, $j_{\lambda(i)}$, which are functions of both temperature and density, were determined using the five-level atom approximation and updated atomic data reported in Berg et al. (2015).

Typically, the total oxygen abundance $(\mathrm{O} / \mathrm{H})$ in an $\mathrm{H}$ II region is calculated by simply summing the $\mathrm{O}^{+} / \mathrm{H}^{+}$ and $\mathrm{O}^{+2} / \mathrm{H}^{+}$ionic abundances, as contributions from $\mathrm{O}^{+3} / \mathrm{H}^{+}$(requiring an ionization energy of $54.9 \mathrm{eV}$ ) are thought to be negligible. Although no emission is seen for the strongest UV O IV transitions $(\lambda \lambda 1401,1404)$ in the LRIS spectrum, the presence of He II from recombination (requiring an ionization energy of $54.4 \mathrm{eV}$ ) makes this argument less secure. To account for possible contributions from $\mathrm{O}^{+3}$, we measured the $3 \sigma$ upper limit on the flux at $\lambda \lambda 1401,1404$ to be $\leq 3.3 \times 10^{-18} \mathrm{erg} \mathrm{s}^{-1}$ $\mathrm{cm}^{-2}$. Comparing this value to the $\mathrm{O}$ III] $\lambda \lambda 1661,1666$ line strengths, we find a marginal oxygen ionization correction factor (ICF) of 1.055 .

For SL2S0217 we can measure $\mathrm{O}^{+2} / \mathrm{H}^{+}$from the [O III] $\lambda \lambda 4959,5007 / \mathrm{H} \beta$ optical emission line ratio, however
Ionic and Relative Nebular Abundances

\begin{tabular}{|c|c|c|}
\hline$n_{e}$ used & $100 \mathrm{~cm}^{-3}$ & $4,500 \mathrm{~cm}^{-3}$ \\
\hline 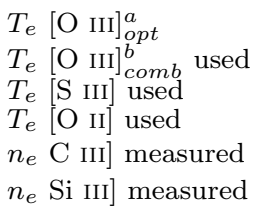 & $\begin{array}{r}15,700 \pm \\
15,400= \\
14,500= \\
13,800= \\
300_{-30}^{+1,30} \\
4,500_{-1,}^{+1,}\end{array}$ & $\begin{array}{l}3,200 \mathrm{~K} \\
\pm 200 \mathrm{~K} \\
\pm 200 \mathrm{~K} \\
\pm 200 \mathrm{~K} \\
50 \mathrm{~cm}^{-3} \\
000 \mathrm{~cm}^{-3} \\
400 \mathrm{~cm}^{-3}\end{array}$ \\
\hline $\begin{array}{l}\mathrm{O}^{+} / \mathrm{H}^{+a, c} \\
\mathrm{O}^{+2} / \mathrm{H}^{+a} \\
\mathrm{ICF}^{d} \\
12+\log (\mathrm{O} / \mathrm{H})^{a}\end{array}$ & $\begin{aligned} 5.21 & \times 10^{-7} \\
3.14 \pm 0.12 & \times 10^{-5} \\
& \geq 7.50^{1.0}\end{aligned}$ & $\begin{array}{l}8.77 \times 10^{-7} \\
3.13 \pm 0.12 \times 10^{-5} \\
55 \\
\geq 7.51^{e}\end{array}$ \\
\hline $\begin{array}{l}\mathrm{C}^{+3} / \mathrm{C}^{+2} \\
\mathrm{C}^{+2} / \mathrm{O}^{+2} \\
\log \mathrm{U} \\
\mathrm{ICF} \\
\log (\mathrm{C} / \mathrm{O})\end{array}$ & $\begin{array}{r}0.86 \\
0.122 \pm 0.005 \\
-1 \\
1.27 \pm \\
-0.81 \pm 0.09\end{array}$ & $\begin{array}{l}0.86 \\
0.124 \pm 0.005 \\
.50 \\
0.27 \\
-0.80 \pm 0.09\end{array}$ \\
\hline $\begin{array}{l}\mathrm{N}^{+2} / \mathrm{O}^{+2} \\
\log (\mathrm{N} / \mathrm{O})\end{array}$ & $\begin{array}{r}0.033 \pm 0.062 \\
-1.48 \pm 0.46\end{array}$ & $\begin{array}{l}0.029 \pm 0.054 \\
-1.53 \pm 0.45\end{array}$ \\
\hline $\begin{array}{l}\mathrm{Si}^{+2} / \mathrm{C}^{+2} \\
\mathrm{ICF} \\
\log (\mathrm{Si} / \mathrm{C}) \\
\log (\mathrm{Si} / \mathrm{O})\end{array}$ & $\begin{array}{r}0.072 \pm 0.002 \\
2.31 \pm \\
-0.76 \pm 0.09 \\
-1.59 \pm 0.17\end{array}$ & $\begin{array}{l}0.073 \pm 0.002 \\
\qquad 0.32 \\
\quad-0.77 \pm 0.09 \\
-1.58 \pm 0.17\end{array}$ \\
\hline
\end{tabular}

Note. - Physical conditions and abundances derived for the nebular gas in SL2S0217 assuming either $n_{e}=100 \mathrm{~cm}^{-3}$ (consistent with the $n_{e}$ from C III]) or $n_{e}=4,500 \mathrm{~cm}^{-3}$ (from Si III]). Ionic abundances are reported for both the optical and UV species when available.

${ }^{a}$ Determined from the $H S T$ optical grism spectrum values.

${ }^{b}$ Determined from the combined UV and optical $\lambda 1666 / \lambda 5007$ line ratio.

${ }^{c}$ The $[\mathrm{O}$ II $] \lambda 3727$ emission line intensity was inferred from the observed [O III $\lambda 5007$ flux and the [O III $] \lambda 5007 /\left[\begin{array}{l}\mathrm{OII}\end{array} \lambda 3727\right.$ ratio from CLOUDY photoionization modeling.

${ }_{d}$ The oxygen ICF accounts for unseen $\mathrm{O}^{+3}$, estimated from the $3 \sigma$ flux upper limit of O IV] $\lambda \lambda 1401,1404$.

${ }^{e}$ Because we do not detect [O II], the estimated oxygen abundance is reported as a lower limit. However, the $\mathrm{O}^{+}$contribution is expected to be small at such high ionization.

the corresponding lines for $\mathrm{O}^{+} / \mathrm{H}^{+}$are not detected in the optical grism spectrum. In order to estimate $12+\log (\mathrm{O} / \mathrm{H})$ from the optical spectrum, we used the observed [O III] $\lambda 5007$ emission and results from photoionization models (see Section 8.1) to predict the [O II] $\lambda 3727$ emission line flux. Based on these models, $\mathrm{O}^{+} / \mathrm{H}^{+}$ only contributes $2 \%$ to the total oxygen abundance (and $\mathrm{O}^{0}$ is truly negligible), but such a small contribution is expected at the extreme ionization level of SL2S0217. Therefore, we find SL2S0217 to be an extremely metal poor $(\mathrm{EMP} ; 12+\log (\mathrm{O} / \mathrm{H})<7.7)$ galaxy, with an oxygen abundance, corrected for the unseen $\mathrm{O}^{+} / \mathrm{H}^{+}$and $\mathrm{O}^{+3} / \mathrm{H}^{+}$contributions, with $12+\log (\mathrm{O} / \mathrm{H})=7.50$. The ionic and total oxygen abundances are listed in Table 8 .

\subsection{1. $C / O$ Abundance}

Abundance determinations for other elements require ionization correction factors to account for their unobserved ionic species. The prominence of the highionization-potential He II and C IV emission lines observed in SL2S0217 indicate that ICFs are particularly important for C. Although the relatively narrow emission profiles of the C IV doublet indicate the dominance 
of nebular emission, it is complicated by potential contributions from a range of stellar features, from pure absorption to P-Cygni profiles. To avoid this complication, we used the ICF presented in Berg et al. (2016) to estimate the $\mathrm{C}^{+3}$ contribution at $\log \mathrm{U}=-1.50$ (our best model value; see Section 8.1). Despite the strong C IV emission present in the UV spectrum of SL2S0217, we determine a modest $\mathrm{C} \mathrm{ICF}=1.27 \pm 0.27$. The $\mathrm{C} / \mathrm{O}$ abundance is then determined using

$$
\frac{\mathrm{C}}{\mathrm{O}}=\frac{\mathrm{C}^{+2}}{\mathrm{O}^{+2}} \times \mathrm{ICF}
$$

to be $\log (\mathrm{C} / \mathrm{O})=-0.81 \pm 0.03$, which is typical of starforming dwarf galaxies with similar oxygen abundance (Garnett et al. 1995b, Berg et al. 2016).

Carbon and oxygen are produced on different time scales, by different mass populations of stars, and so the $\mathrm{C} / \mathrm{O}$ trend with metallicity has been studied by many authors with observations for a variety of astrophysical objects, and using a variety of chemical evolution models and stellar yields. Oxygen is synthesized by SNe II (massive stars) and returned to the interstellar medium quickly, whereas carbon is primarily produced by $\mathrm{He}$ burning through the triple- $\alpha$ process, a reaction that can occur in both massive $\left(M>8 M_{\odot}\right)$ and low to intermediate mass $\left(1 M_{\odot}<M<8 M_{\odot}\right)$ stars.

Historically, $\mathrm{C} / \mathrm{O}$ has been difficult to measure, but recently $\mathrm{C} / \mathrm{O}$ has been measured in dozens of nearby, metal-poor, dwarf galaxies using the UV lines (e.g., Berg et al. 2016; Senchyna et al. 2017). Similar to the early work of Garnett et al. (1995b), these studies find a general trend of increasing $\mathrm{C} / \mathrm{O}$ with oxygen abundance, but with a significant unexplained scatter. These data can also be interpreted as a constant trend in $\mathrm{C} / \mathrm{O}$ at low-metallicities. We plot SL2S0217 (open star) on this $\mathrm{C} / \mathrm{O}$ versus $\mathrm{O} / \mathrm{H}$ trend in the left panel of Figure 10 where nearby dwarf galaxies are plotted as filled circles for comparison. The trend is extended to higher oxygen abundances by incorporating $\mathrm{C} / \mathrm{O}$ determinations from optical recombination lines (filled squares: Esteban et al. 2002, Esteban et al. 2009; Esteban et al. 2014; Pilyugin \& Thuan 2005: García-Rojas \& Esteban 2007; LópezSánchez et al. 2007).

Significant UV C and O has also been measured for a handful of $z \sim 2-3$ galaxies (gold triangles in Figure 10; Pettini et al. 2000; Fosbury et al. 2003; Erb et al. 2010 Christensen et al. 2012 stark et al. 2014 Bayliss et al. 2014; James et al. 2014; Vanzella et al. 2016; Steidel et al. 2016; Amorín et al. 2017; Rigby et al. 2017), amongst which the $\mathrm{C} / \mathrm{O}$ ratio of SL2S0217 appears to be average. Interestingly, $\mathrm{C} / \mathrm{O}$ values for the $z \sim 2-3$ galaxies are consistent with the nearby dwarf galaxies of similar metallicity, but potentially with a lower average value. The distinction between these populations of different ages may support the notion put forth by Berg et al. (2016) that higher $\mathrm{C} / \mathrm{O}$ values may be due to a delayed pseudo-secondary carbon contribution from lowand intermediate-mass stars.

\subsubsection{Relative $N$ Abundance}

We estimate the $\mathrm{N} / \mathrm{O}$ ratio by combining the UV O III] $\lambda \lambda 1661,1666$ and $\mathrm{C}$ III] $\lambda \lambda 1907,1909$ emission lines and the N III] $\lambda 1750$ multiplet. Since the N III] $\lambda 1750$ fea- ture is only weakly detected in the SL2S0217 LRIS spectrum, it is appropriate to think of the following N/O determination as an upper limit. Utilizing the fact that the ionization potentials of $\mathrm{N}^{++}$and $\mathrm{C}^{++}$are nearly the same (47.448 eV and $47.887 \mathrm{eV}$ respectively), we used the $\mathrm{N}^{++} / \mathrm{C}^{++}$ion ratio to measure a relative $\mathrm{N} / \mathrm{C}$ enrichment of $\log (\mathrm{N} / \mathrm{C})=-0.68 \pm 0.02$. Then, combining this ratio with the ionization corrected $\mathrm{C} / \mathrm{O}$ ratio (see Table 8), we inferred a total $\mathrm{N} / \mathrm{O}$ of $\log (\mathrm{N} / \mathrm{O}$ ) $=-1.48 \pm 0.46$, which is consistent with the range of $\log (\mathrm{N} / \mathrm{O})$ seen for local dwarf galaxies with similar oxygen abundance (e.g., van Zee \& Haynes 2006; Berg et al. 2012 ).

Relative N/O abundances are well studied in nearby dwarf and spiral galaxies, providing a robust sample to compare measurements from more distant targets. In the upper right panel of Figure 10 we compare the N/O of SL2S0217 to nearby dwarf (red points: van Zee \& Haynes (2006); Berg et al. (2012); Berg et al. (2016); Berg et al. 2018, in prep.) and spiral galaxies (blue points: the CHAOS project - Berg et al. (2015); Croxall et al. (2015, 2016); Berg et al. 2018, in prep.), as well as nearby galaxies with oxygen abundance measurements from recombination lines (green squares). SL2S0217 lies amongst the $\mathrm{N} / \mathrm{O}$ range of both nearby dwarf galaxies and the handful of $z \sim 2-3$ galaxies with $\mathrm{N} / \mathrm{O}$ and $\mathrm{O} / \mathrm{H}$ measurements (gold triangles). Note that $\mathrm{N} / \mathrm{O}$ value for SL2S0217 falls on the plateau expected for primary (metallicity-independent) $\mathrm{N}$ production, as expected for a low oxygen abundance of $12+\log (\mathrm{O} / \mathrm{H}) \sim 7.5$ (see, for example, the discussion in Pettini et al. 2002a; Pettini \& Cooke 2012).

In the bottom half of Figure 10 (b) we examine the $\mathrm{C} / \mathrm{N}$ ratio, noting the remarkably flat trend for both nearby and distant galaxies. Berg et al. (2016) found that the constant ratio of $\mathrm{C} / \mathrm{N}$ seen over a large range in $\mathrm{O} / \mathrm{H}$ for nearby galaxies may indicate that carbon is predominantly produced by similar nucleosynthetic mechanisms as nitrogen (also see Steidel et al. 2016). However, a larger sample of $\mathrm{C} / \mathrm{N}$ measurements in distant galaxies is needed to determine whether the hypothesis of Berg et al. (2016) also applies at $z \sim 2-3$, or whether different nucleosynthetic processes dominate $\mathrm{C}$ and $\mathrm{N}$.

Recently, observations of the standard rest-frame optical emission-line $[\mathrm{O}$ III $] / \mathrm{H} \beta$ versus $[\mathrm{N}$ II $] / \mathrm{H} \alpha$ diagnostic plot (the BPT diagram; Baldwin et al. 1981) has revealed a pronounced offset of $\sim 2-3$ galaxies from the main sequence of local star forming galaxies (e.g., Masters et al. 2014, Steidel et al. 2014; Shapley et al. 2015). Several studies have investigated the physical origin of this offset by analyzing the BPT parameter space in terms of other physical properties for large surveys of local galaxies. Using $\sim 100,000$ galaxies from the Sloan Digital Sky Survey Data Release 12, Masters et al. (2016) found that the region of the BPT occupied by $\sim 2-3$ galaxies corresponds to galaxies with elevated SFR surface densities, stellar masses, and $\mathrm{N} / \mathrm{O}$ ratios, concluding that the higher $\mathrm{N} / \mathrm{O}$ ratios at fixed $[\mathrm{O}$ III $] / \mathrm{H} \beta$ are the proximate cause of the offset. In contrast, the sample of $z \sim 2-3$ galaxies plotted in Figure 10 occupy the same range in $\mathrm{N} / \mathrm{O}$ as the local comparison sample. SL2S0217, in particular, has an average $\mathrm{N} / \mathrm{O}$ abundance relative to the local dwarf galaxies, however, this value is an upper limit estimated from the weak detection of the $\mathrm{N}$ III] $\lambda 1750$ 

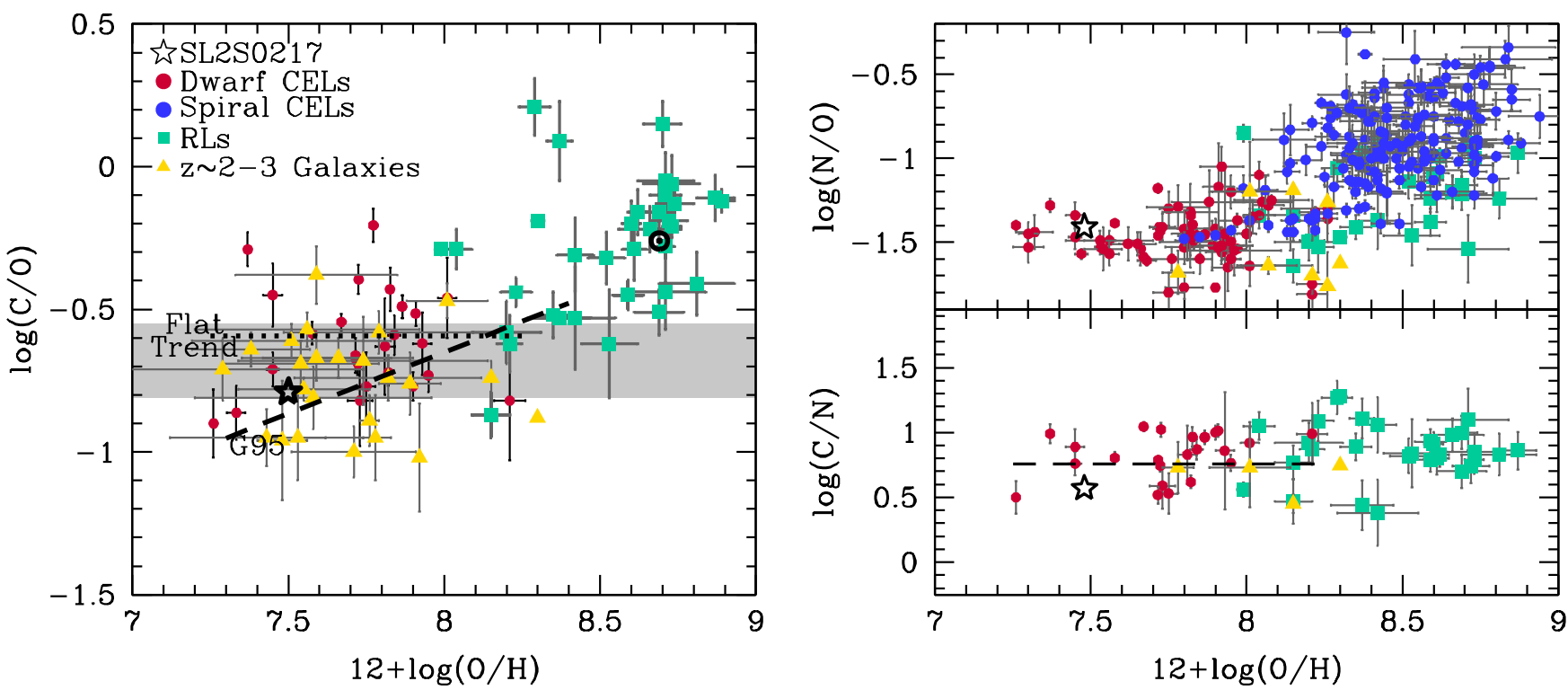

FIG. 10.- Left: Carbon to oxygen ratio vs. oxygen abundance of SL2S0217 (black star) in comparison to other star forming galaxies. Comparison data of nearby galaxies is divided into two groups. Red circles are metal-poor dwarf galaxies as reported in Berg et al. (2016) and Berg et al. (2018, in prep.), and green filled squares represent star-forming galaxies with larger oxygen abundances determined trom recombination lines (Esteban et al. 2002, Esteban et al. 2009 Esteban et al. 2014 Pilyugin \& Thuan 2005 García-Rojas \& Esteban 2007 López-Sánchez et al. 2007). The dashed line is the least-squares fit from Garnett et al. (1995b. G95) and the dotted line is the weighted mean of the significant CEL C/O detections (filled red circles). More distant $(z \sim 2-3)$ galaxies are represented as gold triangles (Pettini et al. 2000 Fosbury et al. 2003. Erb et al. 2010. Rigby et al. 2011. Christensen et al. 2012. Stark et al. 2014 Bayliss et al. 2014 James et al. 2014 Vanzella et al. 2016 Steidel et al. 2016 Amorín et al. 2017 Rigby et al. 2017), including composite spectra from Steidel et al. (2016), Amorin et al. (2017), and Rigby et al. (2017). The background shaded region demonstrates the value for the $z \sim 3$ Lyman break galaxy composite spectrum determined by Shapley et al. (2003).

Right: Trends in relative nitrogen abundance versus oxygen abundance for galaxies spanning a range in redshift. Additional direct abundances are plotted for nearby dwarf galaxies (van Zee \& Haynes 2006 Berg et al. 2012) and for nearby spiral galaxies (blue points: the CHAOS project - Berg et al. (2015); Croxall et al. (2015, 2016); Berg et al. (2018), in prep.). SL2S0217 appears to have a normal N abundance with respect to both nearby, metal-poor dwart galaxies and the few other $z \sim 2-3$ galaxies with $\mathrm{C} / \mathrm{N}$ observations.

line. Further, despite the unusual strength of its UV and optical emission lines, the $[\mathrm{O} \mathrm{III}] / \mathrm{H} \beta$ and $[\mathrm{N} \mathrm{II}] / \mathrm{H} \alpha$ (inferred from photoionization modeling; $\S 8.1 .1$ ratios of SL2S0217 indicate it may not coincide with the offset region of the BPT. Clearly there is still much to understand about the $z \sim 2-3$ BPT diagram.

\subsubsection{Relative Si Abundance}

Silicon abundances relative to carbon were determined following the logic presented in Garnett et al. (1995a): The Si III] and C III] doublets arise from comparable energy levels ( $\sim 6.57$ and $6.50 \mathrm{eV}$ respectively), and therefore the $\mathrm{Si} / \mathrm{C}$ abundance ratio is relatively insensitive to uncertainties in the electron temperature. Additionally, $\mathrm{Si} / \mathrm{C}$ depends little on the assumed density as both Si III] and $\mathrm{C}$ III] have very high critical densities, meaning their volume emissivities have a similar dependence on density. Finally, the proximity of the Si III] and C III] emission lines in wavelength eliminates strong effects of differential extinction.

The Si III] and C III] emission line features are extremely prominent in SL2S0217; we measured their strengths with $\mathrm{S} / \mathrm{N}>20$ (see Figure 5). As described in Section 7.1.2, we assume the ionized gas of SL2S0217 is in the low density limit $\left(<10^{3} \mathrm{~cm}^{-3}\right)$, where collisional de-excitation does not significantly impact the $\mathrm{Si}^{+2} / \mathrm{C}^{+2}$ ratio derived from the UV lines. Because $\mathrm{C}^{+2}$ has a larger ionization potential than $\mathrm{Si}^{+2}(47.9$ and $33.5 \mathrm{eV}$ respectively), we expect a larger fraction of $\mathrm{Si}$ to be in a higher, unobserved ionization state than $\mathrm{C}$, and so an ICF is needed to determine the $\mathrm{Si} / \mathrm{C}$ abundance. We determine the $\mathrm{Si} / \mathrm{C}$ element abundance using the equation

$$
\frac{\mathrm{Si}}{\mathrm{C}}=\frac{\mathrm{Si}^{+2}}{\mathrm{C}^{+2}}\left[\frac{X\left(\mathrm{Si}^{+2}\right)}{X\left(\mathrm{C}^{+2}\right)}\right]^{-1}=\frac{\mathrm{Si}^{+2}}{\mathrm{C}^{+2}} \times \mathrm{ICF},
$$

where $\mathrm{X}\left(\mathrm{Si}^{+2}\right)$ and $\mathrm{X}\left(\mathrm{C}^{+2}\right)$ are the $\mathrm{Si}^{+2}$ and $\mathrm{C}^{+2}$ volume fractions, respectively. The ICF is modeled as a function of the ionization parameter using the photoionization code CLOUDY (Ferland et al. 2013), with BPASS models as the input ionizing radiation field (see Section 8.1). We sought simple models that reproduced the conditions in SL2S0217 as closely as possible; see Section 8.1 for a detailed discussion.

The ionization fraction of $\mathrm{Si}$ and $\mathrm{C}$ species as a function of ionization parameter are shown in Figure 11. As we will show in Section 8.1.1, we can reproduce many aspects of the observed spectrum using CLOUDY models of a stellar population with binaries and an ionization parameter of $\log \mathrm{U}=-1.5$; this value is reasonable given the very hard ionizing radiation field predicted by the SED modeling of B12. We estimate the uncertainty in the ICF as the scatter amongst the different models considered at a given $\mathrm{C}^{+2}$ volume fraction. The resulting $\mathrm{Si}$ $\mathrm{ICF}=2.31 \pm 0.32$ for $12+\log (\mathrm{O} / \mathrm{H})=7.5$ and $t=10^{7.0}$ yrs is significant, correcting the relative $\mathrm{Si}$ abundance from $\log (\mathrm{Si} / \mathrm{C})=-1.14$ to $\log (\mathrm{Si} / \mathrm{C})=-0.76 \pm 0.09$. 

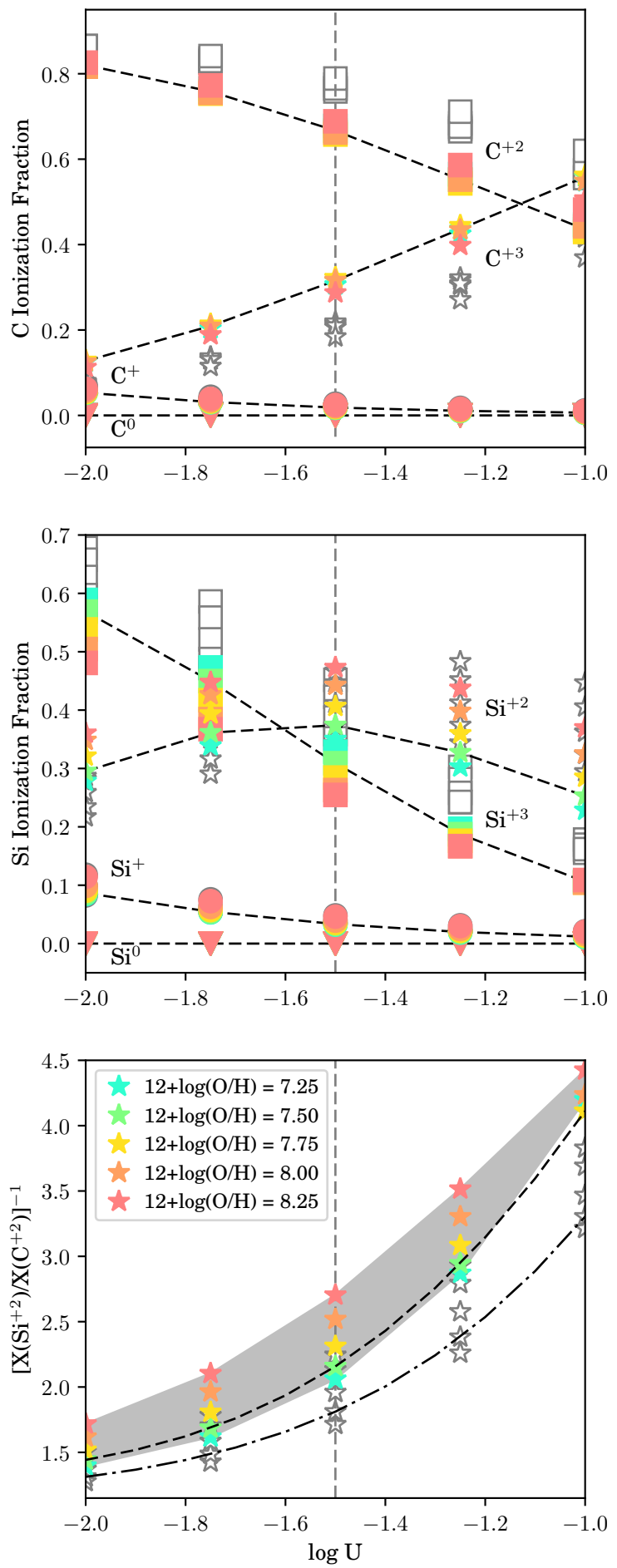

FIG. 11.- Ionization fraction of $\mathrm{C}$ and $\mathrm{Si}$ species as a function of ionization parameter from CLOUDY models of $\mathrm{Z}_{n e b}=0.05 \mathrm{Z}_{\odot}(\mathrm{Z}=$ 0.001) BPASS stellar models. Two different ages of instantaneous bursts are shown, demonstrating the limits of the parameter space explored: $t=10^{6.4}$ and $10^{7.0}$ yrs. For the older model, symbols are color coded by the gas-phase oxygen abundance, whereas the younger model is depicted by gray symbols. In the top two panels the different ionic species are designated by triangles, circles, squares, and stars in order of increasing ionization. The bottom panel plots the ionization correction factor (ICF) vs. ionization parameter, where the dashed and dashed-dotted curves indicate the ICF for the $12+\log (\mathrm{O} / \mathrm{H})=7.5$ model at an age of $10^{6.4}$ and $10^{7.0}$ yrs respectively and the gray shading highlights the range spanned by metallicity. The gray vertical dashed line marks the predicted ionization parameter for SL2S0217.
When combined with the $\mathrm{C} / \mathrm{O}$ abundance derived for SL2S0217, we estimate $\log (\mathrm{Si} / \mathrm{O})=-1.59 \pm 0.17$. The relevant $\mathrm{Si}$ abundance values are reported in Table 8.

Due to the need of far-UV spectroscopic observations of nearby galaxies, there exists a general paucity of nebular measurements of Si/O to compare to. Perhaps the bestknown study of Si/O comes from Garnett et al. (1995a), who measured the UV Si III] emission lines for seven extragalactic H II regions using the Faint Object Spectrograph on $H S T$. These authors found $\mathrm{Si} / \mathrm{O}$ to be constant over the observed range in oxygen abundance, with a weighted mean value of $\log (\mathrm{Si} / \mathrm{O})=-1.59 \pm 0.07$. Our $\log (\mathrm{Si} / \mathrm{O})=-1.59 \pm 0.17$ measurement for SL2S0217 is remarkably consistent with this average from the Garnett et al. (1995a) study, but indicates significant $\mathrm{Si} / \mathrm{O}$ enrichment or a reduced Si depletion onto dust relative to the $z \sim 2.4$ galaxies of Steidel et al. $(2016, \log (\mathrm{Si} / \mathrm{O})$ $=-1.81 \pm 0.10)$.

Note that our $\mathrm{Si} / \mathrm{O}$ value does not account for the relative depletion of $\mathrm{Si}$ and $\mathrm{O}$ onto dust grains. While one might expect this effect to be small given the low predicted dust reddening in SL2S0217 and the probability of grain destruction or erosion in the presence of a hard ionizing radiation field, our $\mathrm{Si} / \mathrm{O}$ value is significantly lower than the solar ratio of $\log (\mathrm{Si} / \mathrm{O})_{\odot}=-1.15($ Jenkins 2009). Many studies argue that both $\mathrm{Si}$ and $\mathrm{O}$ are predominantly products of massive star nucleosynthesis, and so their ratio is expected to vary little with time (e.g., Timmes et al. 1995). Note, however, that some studies find Si production can have a substantial contribution from Type Ia SNe outbursts (c.f., Matteucci \& Greggio 1986, Kobayashi et al. 2006). Since oxygen doesn't deplete as strongly, the observed underabundance can likely be attributed to the depletion of Si onto dust (Steidel et al. 2016).

We estimated the logarithmic depletion of $\mathrm{Si}$ in the ISM following the prescription laid out in Jenkins $(2009)^{8}$ :

$$
[\mathrm{Si} / \mathrm{H}]_{\text {gas }}=[\mathrm{Si} / \mathrm{H}]_{\mathrm{obs}}-[\mathrm{Si} / \mathrm{H}]_{\mathrm{ref}}
$$

where, assuming the observed subsolar $\mathrm{Si} / \mathrm{O}$ ratio is solely due to $\mathrm{Si}$ depletion, we use $\mathrm{Si} / \mathrm{O}$ in place of $\mathrm{Si} / \mathrm{H}$ and use the solar values from Asplund et al. (2009) for our reference. This results in a dust depletion of $[\mathrm{Si} / \mathrm{H}]_{\text {gas }}=-0.43$ for SL2S0217; a value typical with respect to Garnett et al. (1995a), but smaller than the value derived by Steidel et al. (2016). From observations of nearby dwarf galaxies we have come to expect [Si/Fe] enrichment at low $[\mathrm{Fe} / \mathrm{H}]$ due to early contamination of Si by Type II SNe. However, this exercise indicates that a significant fraction of the Si produced in SL2S0217 is missing, perhaps resulting in the subsolar $\mathrm{Si} / \mathrm{Fe}$ ratio measured from the absorption lines (Section 8).

Jenkins (2009) interpreted the abundances of 17 different elements from more than 100 studies of sight lines to 243 different stars to construct a unified representation

8 More recently, Jenkins \& Wallerstein (2017) updated the elemental depletion equations for a given sightline in the Large Magellanic Cloud, including updated parameters for the Si depletion. However, this work does not analyze the depletion relationships for $\mathrm{C}, \mathrm{N}$, or O. Therefore, we have used the Jenkins (2009) equations for consistency.

${ }^{9}$ For dust depletion of $\mathrm{D}(\mathrm{x})=\left[\frac{X}{H}\right]$, the dust depletion factor is $\delta(\mathrm{x})=1-10^{\mathrm{D}(\mathrm{x})}$. 
of depletions that is linearly dependent on the severity of depletion along a given sight line, $F_{*}$. Using this model and the inferred depletion value of $\mathrm{Si}$, we estimated the depletion of the other significant elements in the SL2S0217 UV spectrum - C, N, and O. Using the fit from Jenkins $(2009)$ for $\mathrm{Si},[X / \mathrm{H}]_{\text {gas }}=B_{X}+A_{X}\left(F_{*}-z_{X}\right)(\mathrm{co}-$ efticients reported in their Table 4$)$, we found a depletion severity of $F_{*}=0.182$. Then, we determine the following dust depletion factors: $\delta(\mathrm{Si})=0.628, \delta(\mathrm{C})=0.259$, $\delta(\mathrm{N})=0.222$, and $\delta(\mathrm{O})=0.111^{9}\left([\mathrm{C} / \mathrm{H}]_{\text {gas }}=-0.130\right.$, $[\mathrm{N} / \mathrm{H}]_{\text {gas }}=-0.109$, and $\left.[\mathrm{O} / \mathrm{H}]_{\text {gas }}=-0.051\right)$. While these depletion values imply a systemically higher relative $\mathrm{Si} / \mathrm{O}$ abundance $(\Delta \sim 0.4 \mathrm{dex})$, the $\mathrm{O} / \mathrm{H}, \mathrm{C} / \mathrm{O}$, and $\mathrm{N} / \mathrm{O}$ ratios would remain relatively unchanged $(\Delta \sim 0.05$ dex).

\section{DISCUSSION}

\subsection{Photoionization Models}

While the number of objects with detections of extreme UV emission line features continues to grow, many questions remain regarding their ionizing sources. To gain insight, we investigated the potential to produce strong UV emission lines from an ionizing radiation field powered by stars. Previous studies have also used photoionization models to examine the factors that regulate UV emission lines, focusing on $\mathrm{C}$ III] in particular. Stark et al. (2014) found that metal-poor $\left(Z_{n e b}<0.4 Z_{\odot}\right)$, young $(<50 \mathrm{Myr})$ galaxies with subsolar $\mathrm{C} / \mathrm{O}$ ratios and large ionization parameters were capable of reproducing the large C III] EWs observed for high-redshift galaxies. More recently, Jaskot \& Ravindranath (2016) created a large grid of photoionization models of starforming galaxies to examine $\mathrm{C}$ III] EWs by varying starburst age, metallicity, ionization parameter, $\mathrm{C} / \mathrm{O}$ abundance, dust content, gas density, optical depth, and nebular geometries. These authors found that low metallicities and high ionization parameters enhance C III] emission. Further, the largest C III] EWs were produced by stellar populations that incorporated binary interactions among massive stars. Note, however, that models that include stellar rotation have also been shown to extend the lifetime (up to $5-7 \mathrm{Myr}$ ) and increase the strength of nebular emission (e.g., Byler et al. 2017, Choi et al. 2017). We focus here on models using binaries alone, as they can produce nebular emission up to $\sim 10 \mathrm{Myr}$, but note that likely both rotation and binaries play a role in the observed line strengths.

Our primary goal was to reproduce the strong UV emission line ratios observed for SL2S0217. Building on the lessons of Jaskot \& Ravindranath (2016), we ran a grid of photoionization models using CLOUDY17.00 in which ionization parameter, gas-phase metallicity, and relative $\mathrm{C}, \mathrm{N}$, and $\mathrm{Si}$ abundances were varied. For the input ionizing radiation field, we used a suite of models from the new release of "Binary Population and Spectral Synthesis" (BPASSv2.14; Eldridge \& Stanway 2016 Stanway et al. 2016), with an initial mass function $(\mathrm{MMF})$ index of -1.30 for $0.1-0.5 M_{\odot}$ and -2.35 for $M>0.5 M_{\odot}$.

We ran six sets of models depending on three variables: whether (1) an IMF upper mass cutoff of 100 or $300 M_{\odot}$ was used for $(2)$ continuous or a burst of star formation, and (3) whether or not binary evolution was included. Limited parameter ranges were informed by the SED modeling of Brammer et al. (2012) for the stellar population properties and by the UV emission line ratios measured from the LRIS spectrum for the nebular gas properties. In particular, Jaskot \& Ravindranath (2016) found that C III] EWs as strong as those measured for SL2S0217 (>10 ̊) only result from a hard radiation field (log $\mathrm{U}>-2)$. Each model set considered an age range of $10^{6.4}-10^{7.0} \mathrm{yrs}$, a range in ionization parameter of $-2.0<\log \mathrm{U}<-1.0$, with stellar metallicity of $\mathrm{Z}=0.001\left(\mathrm{Z}_{\star}=0.05 \mathrm{Z}_{\odot}\right)$ to match the estimated gas-phase abundance $(12+\log (\mathrm{O} / \mathrm{H})=7.5$ or $\left.\mathrm{Z}_{n e b}=0.065 \mathrm{Z}_{\odot}\right)$. The GASS10 solar abundance ratios within CLOUDY were used to initialize the relative gas-phase abundances. These abundances were then scaled to cover a range in nebular metallicity $(7.25<$ $12+\log (\mathrm{O} / \mathrm{H})<8.25)$, and relative $\mathrm{C}, \mathrm{N}$, and $\mathrm{Si}$ abundances $\left(0.1<(\mathrm{X} / \mathrm{O}) /(\mathrm{X} / \mathrm{O})_{\odot}<0.5\right)$. The range in relative $\mathrm{N} / \mathrm{O}, \mathrm{C} / \mathrm{O}$, and $\mathrm{Si} / \mathrm{O}$ abundances was motivated by our measured values for SL2S0217 and the observed values for nearby metal-poor dwarf galaxies (e.g., Berg et al. 2012, Berg et al. 2016; Garnett et al. 1999). The resulting $\mathrm{X} / \mathrm{O}=[0.1,0.3,0.5] \mathrm{X} / \mathrm{O}_{\odot}$ model parameters (written as CNSi $=[0.1,0.3,0.5]$ for shorthand) were $\log (\mathrm{C} / \mathrm{O})=$ $[-1.26,-0.78,-0.56], \log (\mathrm{N} / \mathrm{O})=[-1.86,-1.38,-1.16]$, and $\log (\mathrm{Si} / \mathrm{O})=[-2.18,-1.70,-1.48]$.

\subsubsection{Best Model}

To determine the best model from our grid, we first eliminated sets of models that were obviously poor matches. The single-star model sets do not reproduce the UV nebular emission line feature strengths (see also, Jaskot \& Ravindranath 2016), and so were not investigated further. For the young ages considered here $\left(t<10^{7.2} \mathrm{yrs}\right)$, the continuous star formation models are consistent with the burst models, and so only the burst models were examined as representative of both types of star formation. Note, however, that continuous star formation models do produce very different results from bursts for older systems. Finally, the models with an IMF cutoff of $300 M_{\odot}$ do produce more He II ionizing photons than the $100 M_{\odot}$ models, yet they still fall short of producing the He II flux observed for SL2S0217, and significantly overpredict the $\mathrm{Si}$ III]/C III] ratio. Therefore, we employed an error-weighted $\chi^{2}$-minimization routine of the grid of binary burst models with an IMF cutoff of $100 M_{\odot}$. We find we can reproduce most of the features in the UV emission-line spectrum of SL2S0217 with a model similar to the SED modeling of Brammer et al. (2012): the best model is a high-ionization (log $\mathrm{U}=-1.5)$, metal-poor $(12+\log (\mathrm{O} / \mathrm{H})=7.75 ; \mathrm{CNSi}=$ 0.3 ) gas cloud, ionized by $\mathrm{Z}=0.001$ star formation with binaries at an age of $t=10^{7.0}$ yrs.

In Figure 12 we compare the normalized LRIS spectrum of SL2S0217 to the output model spectrum at the spectral resolution of the observed spectrum. The C IV, $\mathrm{O}$ III], N III], Si III], and C III] emission lines are all relatively well-matched, while the He II emission feature is clearly discrepant. Notably, Fosbury et al. (2003) also observed strong, narrow nebular He II and C IV emission from the $z \sim 3.4$ Lynx Arc, reportedly due to a young, low-metallicity stellar cluster with an extreme ionization parameter of $\log \mathrm{U}=-1.0$. However, to date, no pure 


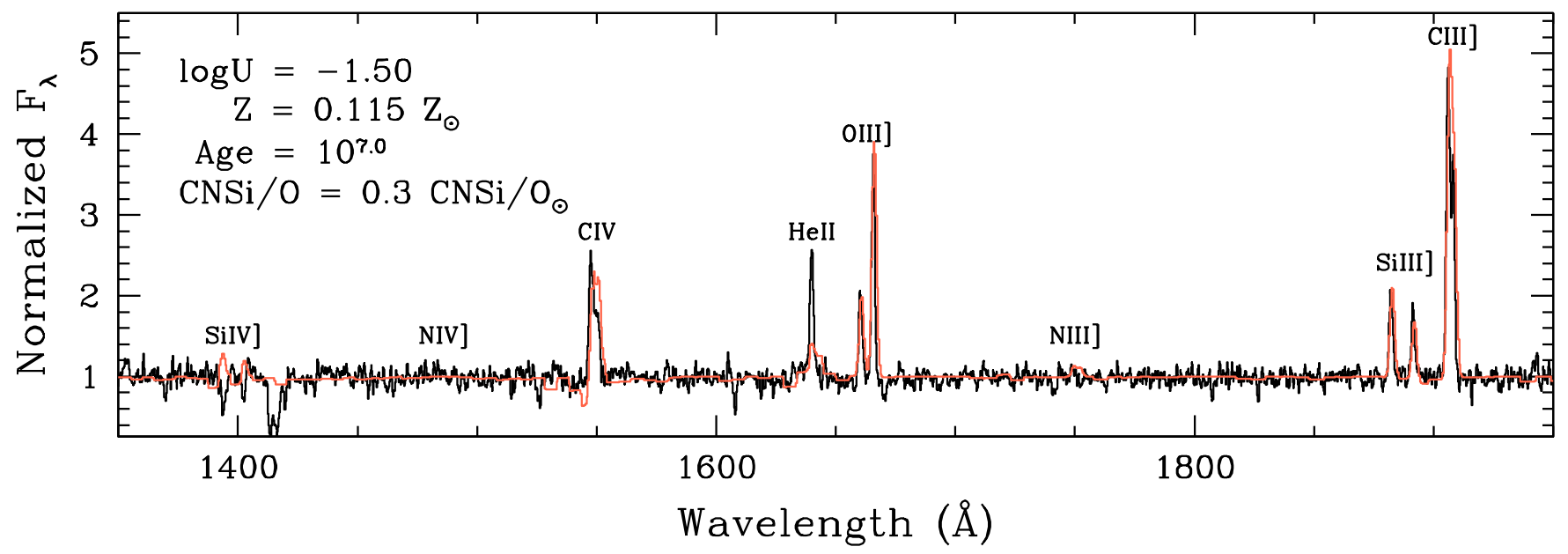

FIG. 12.- The photoionization model (orange line) best-matched to the blue arm of the UV SL2S0217 spectrum. SL2S0217 can be described by a high-ionization $(\log \mathrm{U}=-1.5)$, metal-poor $\left(12+\log (\mathrm{O} / \mathrm{H})=7.75 ; \mathrm{X} / \mathrm{O}=0.3 \mathrm{X} / \mathrm{O}_{\odot}\right)$ gas cloud, ionized by a $\mathrm{Z}=0.001$ star-burst BPASS binary model at an age of $t=10^{7.0} \mathrm{yrs}$. All of the emission features, except He II which is notoriously difficult to emulate with pure photoionization models, are reproduced with this model.

stellar photoionization studies have reported the levels of nebular He II emission observed in SL2S0217 or the Lynx Arc. Interestingly, we do not see the N IV] emission line in our SL2S0217 spectrum despite the fact that we see strong $\mathrm{C}$ IV emission and $\mathrm{C}^{++}$and $\mathrm{N}^{++}$have similar ionization potentials $(47.888 \mathrm{eV}$ and $47.445 \mathrm{eV}$, respectively). The $\mathrm{N} \mathrm{IV]} \mathrm{emission} \mathrm{line} \mathrm{is} \mathrm{also} \mathrm{absent} \mathrm{from} \mathrm{the}$ best model, indicating that $\mathrm{N}$ emission is mostly sensitive to metallicity and, therefore, not expected in metal-poor galaxies like SL2S0217, whereas C IV is more sensitive to temperature / cooling. This fact has motivated the use of the strong-line $[\mathrm{N} \mathrm{II}] / \mathrm{H} \alpha$ metallicity indicator in nearby galaxies, however, the line ratio is not available from the ground at high redshifts $(z \gtrsim 2.5)$ and is highly sensitive to ionization parameter at larger metallicities (e.g., Kewley \& Dopita 2002). Since exceptionally low nitrogen abundances seem to be common amongst distant galaxies with similar extreme emission line features (e.g., Bayliss et al. 2014; James et al. 2014, Smit et al. 2017), the lack of UV N III] and N IV] could help identify additional metal-poor galaxies at high redshifts.

The predicted nebular emission line ratios from our photoionization models are plotted relative to the ratios measured from the LRIS spectrum of SL2S0217 in Figure 13. Different gas-phase metallicity models are indicated by color, where relative $\mathrm{C} / \mathrm{O}, \mathrm{N} / \mathrm{O}$, and $\mathrm{Si} / \mathrm{O}$ abundances increase with point size. The statistically best model is indicated by the dashed yellow line $\left(12+\log (\mathrm{O} / \mathrm{H})=7.75, \mathrm{CNSi}=0.3, t=10^{7.0} \mathrm{yrs}\right)$, where the corresponding yellow-shaded region extends from $t=10^{6.4}-10^{7.0}$ yrs. Note that nebular He II emission peaks before $t=10^{7.0}$ yrs $\left(\sim 10^{6.8} \mathrm{yrs}\right)$ in the models, and so the dashed yellow line in the He II/C III] plot lies within the yellow-shaded region. Interestingly, although C IV also has a very high ionization potential, its nebular line strength continues to increase past $t=10^{7.0}$. Measured line ratios and uncertainties for SL2S0217 are shown as solid black horizontal lines and gray-shaded boxes. Good agreement between model and measurement is found near an ionization parameter of log $\mathrm{U}=-1.50$ for all of the $\mathrm{UV}$ emission line ratios except
He II/C III].

Just as Stark et al. (2014), Berg et al. (2016), and Senchyna et al. (2017) observed strong C III] emission resulting from subsolar $\mathrm{C} / \mathrm{O}$ in metal-poor systems ( $\mathrm{Z}$ $\left.\leq 0.5 \times \mathrm{Z}_{\odot}\right)$, reproducing the observed emission lines in SL2S0217 also requires subsolar C, N, and Si abundance ratios relative to $\mathrm{O}$. The low gas metallicity prevents efficient metal cooling by oxygen, resulting in a high electron temperature that secures a stable production of the collisionally-excited $\mathrm{O}, \mathrm{Si}$, and $\mathrm{C}$ nebular emission lines. If a portion of the metals are also locked up in dust, $\mathrm{C}$ and Si may be under abundant as a result. However, no evidence of dust reddening exists and the extreme ionizing radiation field likely destroyed or eroded the dust in the H II regions (but also see discussion of dust depletion in $\S 7.2 .3$.

\subsection{Stellar Ionization}

The rate of ionizing photons needed to power the arc can be estimated from the demagnified $\mathrm{H} \beta$ flux. At the redshift measured in this work, we calculate a luminosity distance of $D_{l}=1.444 \times 10^{4} \mathrm{Mpc}$ (Wright 2006). The hydrogen ionizing photon luminosity is then given by

$$
Q_{\text {ion. }} \approx \frac{4 \pi D_{l}^{2} F_{H \beta}}{\mu h \nu_{H \beta}} \frac{\alpha_{B}}{\alpha_{H \beta}^{e f f}},
$$

where $\mu$ is the lensing magnification and $\alpha_{B}$ and $\alpha_{H \beta}^{\text {eff }}$ are the total and effective $\mathrm{H} \beta$ case- $\mathrm{B} H$ recombination coefficients. We calculate the recombination coefficients for $T_{e}=1.6 \times 10^{4} \mathrm{~K}$ using the equations of Pequignot et al. (1991). Given the lensing magnification determined here $(\mu=17.3)$ and the $\mathrm{H} \beta$ flux from the grism spectrum, we estimate an ionizing photon luminosity of $\mathrm{Q}_{\text {ion. }}$. = $7.3 \times 10^{53}$ photons $\mathrm{s}^{-1}$. The cluster IMF and metallicity is needed in order to then constrain the nature of the ionizing stellar population, however, this value is still indicative of a large number of massive stars powering the observed spectrum $\left(>10^{3} \mathrm{O}\right.$ stars), which can live to greater ages due to rotation and binary evolution. 

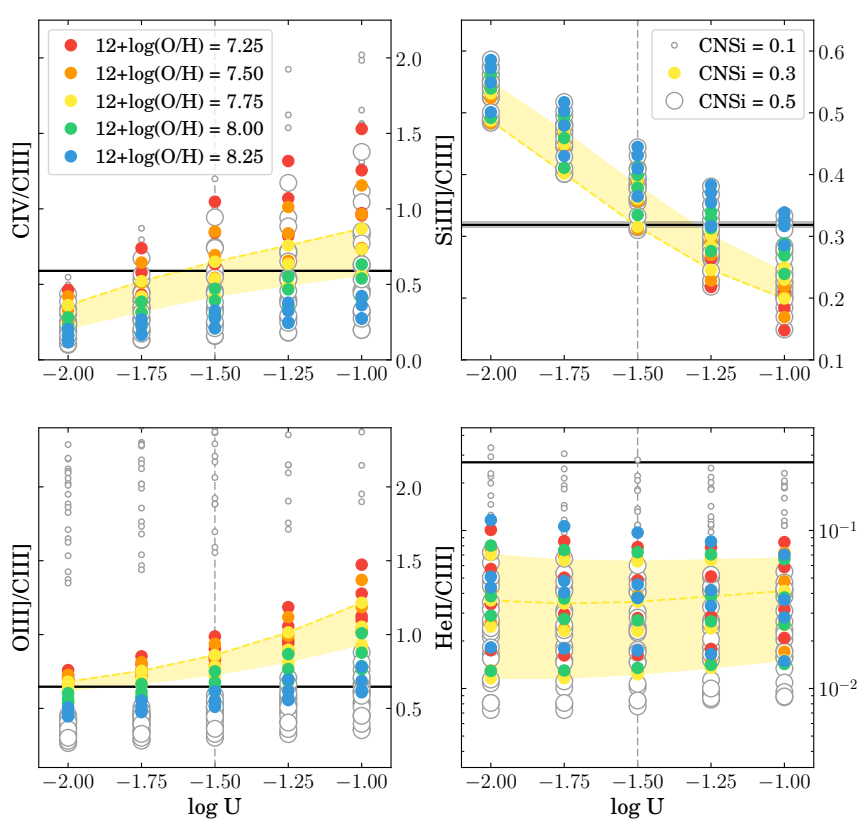

FIG. 13.- UV emission line ratios from CLOUDY photoionization models using the BPASS $\mathrm{Z}_{\star}=0.001 \mathrm{Z}_{\odot}$ binary models for the ionizing source, assuming an $\mathrm{IMF}_{u p}=100 \mathrm{M}_{\odot}$ over a range in age of $10^{6.4}-10^{7.0} \mathrm{yrs}$. The color coded points represent a range of gasphase metallicities assumed: $7.25 \leq 12+\log (\mathrm{O} / \mathrm{H}) \leq 8.25$. We also explored a range in $\mathrm{C} / \mathrm{O}, \mathrm{N} / \mathrm{O}$, and $\mathrm{Si} / \mathrm{O}$ abundances relative to their respective solar ratios, as indicated by various point sizes (see description in Section 8.1. Observed line ratios and corresponding uncertainties for SL2SO217 are plotted as solid black lines and gray extensions, respectively. For the $12+\log (\mathrm{O} / \mathrm{H})=7.75$ models, the change in line ratio as a function of age is shown by the spread of the shaded yellow region, where the dashed yellow line marks the upper limit of $10^{7.0}$ yrs.

\subsection{Multiple Ionization Sources}

While many of the emission-line features observed in the rest-frame UV spectra of SL2S0217 can be reproduced by photoionization from stars alone, outliers such as He II prompted an investigation into additional ionization sources. Indeed, the ratios of rest-frame far-UV emission lines provide a means to discern between nuclear activity and star-formation as the ionizing sources (e.g., Feltre et al. 2016). However, Feltre et al. (2016) uses the photoionization models of Gutkin et al. (2016) which are not appropriate for our young target as they assume continuous star formation for 100 Myrs and do not include the effects of binaries (see Section 8.1.1). Instead, we investigate the effects of incorporating shocks and AGN into the photoionization models presented above.

\subsubsection{Shocks}

Even in star-forming galaxies, a substantial portion of the nebular emission may be generated by shocks (e.g., Krabbe et al. 2014, Rich et al. 2014). This fact motivated Jaskot \& Ravindranath (2016) to incorporate contributions from shocks to their pure photoionization models. In their study, shock+ precursor models were taken from Mappings III (Allen et al. 2008), assuming a metallicity of $Z \sim 0.003$ (the Small Magellanic Cloud models), a velocity range of $125-1000 \mathrm{~km} \mathrm{~s}^{-1}$, and magnetic field strengths of $0.5-10 \mu \mathrm{G}$. The authors find that the addition of the shock models result in an increase of both $\mathrm{C}$ III] and $\mathrm{He}$ II emission relative to $\mathrm{H} \beta$, and, therefore, suggest that nebular He II may serve as a useful diagnostic of shocks for UV spectra.

In light of these findings, we investigated emission from shock ionization as a source for the significant He II emission seen in SL2S0217. Following the methodology of Jaskot \& Ravindranath (2016), we scaled the shock emission and added it to the emission from our photoionization models such that the shocks contribute $10 \%, 30 \%$, $50 \%, 70 \%$, or $90 \%$ of the total predicted emission in $\mathrm{H} \alpha$ (see their Section 2.3 for a more detailed description).

The emission line ratios for a varying shock contribution to our best photoionization model are depicted in Figure 14 (a) relative to those measured for SL2S0217. These results demonstrate that the addition of shocks to the BPASS photoionization does not improve the general match of models to the SL2S0217 features. While the extreme He II/C III] ratio can be reproduced when the ionizing radiation is dominated by shocks, the other observed line ratios are generally over-predicted and cannot be matched with the same conditions. Further, it is physically unlikely that the gas in SL2S0217 is 90\% shock ionized, and so we dismiss shocks as a main source of ionization in SL2S0217.

\subsubsection{Active Galactic Nuclei}

The complex suite of spectral features observed in extreme galaxies such as SL2S0217 may be due to a complex combination of ionizing sources, such as AGN emission diluted by stellar radiation. For super-massive black holes $(\mathrm{SMBH})$ in massive galaxies, numerous studies have found the SMBH mass is well correlated with the velocity dispersion of the host-galaxy bulge (e.g., Gebhardt et al. 2000, Gültekin et al. 2009). However, it is unclear whether this well-known scaling relation extends to the galaxies as small as SL2S0217 (see discussion in Kormendy \& Ho 2013). Black hole masses in dwarf AGNs have been measured in several low-mass star-forming galaxies in the local universe (e.g., Reines et al. 2011, 2014), with the lowest reported mass being just $\sim 50,000 \mathrm{M}_{\odot}$ (Baldassare et al. 2015). Recently, Baldassare et al. (2017) found evidence for dwarf AGN in 11 nearby $(z<0.055)$ composite dwarf galaxies. These authors measured black hole masses ranging from $10^{4.9}-10^{6.1} \mathrm{M}_{B H} / \mathrm{M}_{\odot}$ for galaxies with stellar masses of $10^{8.1}-10^{9.4} \mathrm{M}_{\star} / \mathrm{M}_{\odot}$, on the order of SL2S0217. While these recent studies of local dwarf galaxies indicate that a non-negligible fraction of low-mass galaxies might harbor AGN, we caution that we do not know how these results extrapolate to galaxies at high redshifts.

Given the uncertainties surrounding AGN in low-mass galaxies, it is worthwhile to explore the possibility of producing the extreme emission-line ratios observed in SL2S0217 with the addition of emission from AGN. For this purpose, we used the dust-free, low-metallicity $\left(Z_{\star}=0.25 Z_{\odot}\right)$, narrow-line AGN photoionization models from Groves et al. (2004), assuming simple power-law radiation fields $\left(f_{\nu} \propto \nu^{\beta}\right)$ with a range of powers. As in Section 8.3.1, we scale the AGN emission such that the AGN contribute $10 \%, 30 \%, 50 \%, 70 \%$, or $90 \%$ of the total emission in $\mathrm{H} \alpha$.

Emission line ratios for the best BPASS + AGN models are plotted in Figure 14 (b) relative to those measured for SL2S0217. The resulting shifts in line ratios due to 

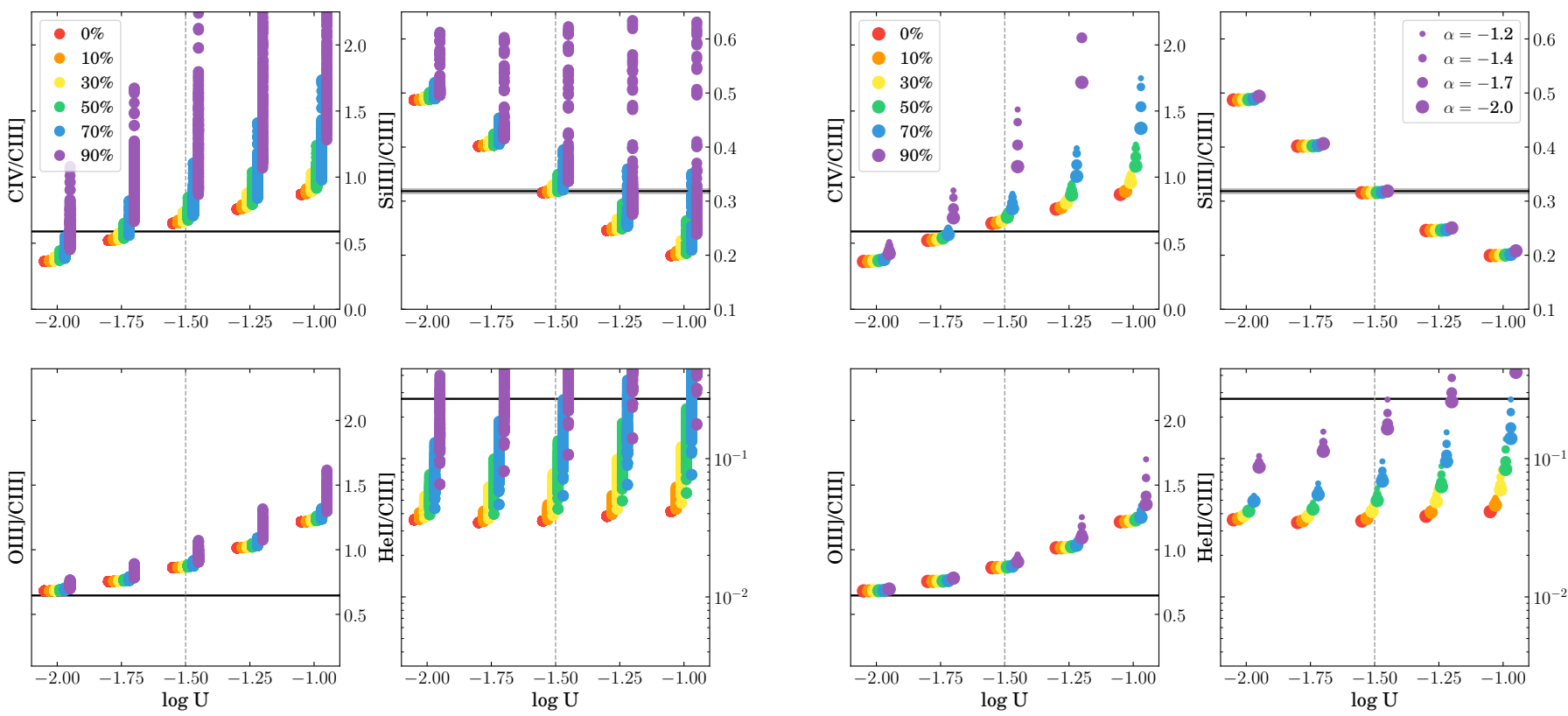

(a) BPASS+Shock Models

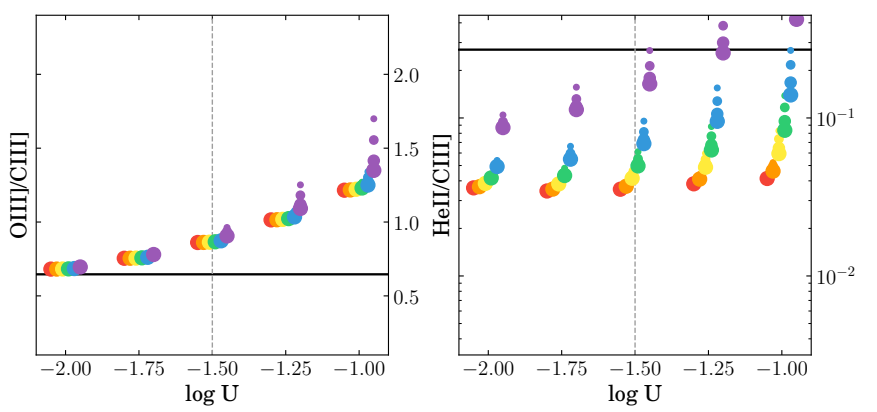

(b) BPASS+AGN Models

FIG. 14.- Cumulative UV emission line ratios from CLOUDY photoionization models plus a (a) shock or (b) AGN contribution, while each color, slightly offset for better visualization, indicates a different fractional contribution from shocks or AGN to the total H $\alpha$ emission. The input stellar photoionization model is the best model fit to the SL2S0217 spectrum (see Figure 12 ). $\mathrm{Z}_{\star} \sim 0.003$ shock+precursor models from Mappings III (Allen et al. 2008) are used for the shock contribution, where the spread represents a range in velocity of $125-1000$ $\mathrm{km} \mathrm{s}^{-1}$ and magnetic field strengths of $0.5-10 \mu \mathrm{G} . \mathrm{Z}_{\star}=0.25 \mathrm{Z}_{\odot}$ dust-free AGN models over a range of power-law exponents (symbol size) are taken from Groves et al. (2004). While the extreme He II/C III] ratio observed for SL2S0217 can be reproduced by models with a significant shock or AGN component, the other line ratios cannot be matched with the same conditions.

the contribution of emission from AGN look very similar to those seen for the BPASS + Shock models. In either case, the additional hard radiation increases the relative emission from the highest ionization lines: He II, O III], and $\mathrm{C}$ IV. This allows the $\mathrm{C}$ IV/C III] ratio to be reproduced with small AGN contributions. Typical narrowline Type II AGN observations have stronger C IV than C III] emission (e.g., Humphreys et al. 2008, Matsuoka et al. 2009: Hainline et al. 2011: Cassata et al. 2013), but our models suggest that the C III] dominant emission in SL2S0217 (C III]/C IV= 1.46) could be produced by a gas-enshrouded AGN.

On the contrary, Figure 14 shows that the $\mathrm{Si}$ III]/C III] ratio is significantly less affected by contributions from AGN than from shocks. This is due, in part, to the fact that the full range of shock velocities are considered at each value of $\log \mathrm{U}$, such that the maximum contribution from shocks for any ion is always included (near $v \sim 175$ $\mathrm{km} \mathrm{s}^{-1}$ for Si III]). On the other hand, the AGN contribution to Si III] emission peaks at low ionization parameter, however, it has little effect on the total Si III] $/ \mathrm{H} \alpha$ emission, which is dominated by photoionization.

An additional difference is seen in the He II/C III] ratio, where the value observed in SL2S0217 is only reached by AGN dominated models ( $>90 \%$ contribution; purple points), yet such models significantly over estimate the $\mathrm{C}$ IV/C III] and $\mathrm{O}$ III]/C III] ratios. Further, if the strong nebular emission lines in SL2S0217 were powered by a Type II AGN, we would also expect to detect several high ionization emission lines that are missing from the rest-frame UV spectrum: N V $\lambda 1240$, Si IV $\lambda 1403$,
N IV $\lambda 1486$ (e.g., Vanden Berk et al. 2001, Hainline et al. 2011). Again, the suite of lines observed for SL2S0217 cannot be simultaneously matched by BPASS photoionization models with an additional source of hard radiation, in this case, from AGN.

\subsection{High Ionization Emission Lines}

While we postulate that SL2S0217 is indeed a starforming galaxy, its strong He II and C IV emission is rare and interesting. In fact, only one percent of UV-selected galaxies at $z \sim 3$ show strong nebular $\mathrm{C}$ IV emission (e.g., Reddy et al. 2008; Hainline et al. 2011; Mainali et al. 2017). In comparison, $z \sim 2-3$ LBGs with large Ly $\alpha$ EWs have been reported to measure relatively weak $\mathrm{C}$ IV emission with strengths $\leq 1 \%$ of $\operatorname{Ly} \alpha$ (e.g., Erb et al. 2010). Notably, significant narrow C IV and/or He II emission are also detected in several nearby dwarf galaxies, suggesting that extremely hard ionizing radiation fields may be common in low-mass, low-metallicity galaxies (e.g., Kehrig et al. 2011; Berg et al. 2016). This idea is further supported by several recent studies that have found nebular C IV and/or He II in distant metalpoor star-forming galaxies (Erb et al. 2010, Christensen et al. 2012; Stark et al.|2014; de Barros et al.|2016; Steidel et al. 2016 Vanzella et al. 2016). Of the few extreme UV emission line galaxies that have been observed at $z \gtrsim 2$ (e.g., Erb et al. 2010, Christensen et al. 2012, Bayliss et al. 2014; Stark et al. 2014, 2015; Caminha et al. 2016; de Barros et al. |2016; Vanzella et al. 2016 : Smit et al. 2017), none show a comparable combination of emission line ratios. 


\subsubsection{Nebular $C$ IV $\lambda \lambda 1548,1550$}

Typically galaxies show $\mathrm{C}$ IV in $(i)$ absorption from the surrounding ISM or CGM gas or (ii) exhibit a P-Cygni profile from the stellar winds of massive stars (e.g., Leitherer et al. 2001). To the contrary, AGN can produce C IV emission, but line-widths are typically broader than nebular emission lines (hundreds of $\mathrm{km} \mathrm{s}^{-1}$ ). To date, narrow C IV has only been observed in a handful of strongly lensed high-redshift galaxies (e.g., Christensen et al. 2012, Stark et al. 2014; Smit et al. 2017), therefore, the significant C IV emission detected in SL2S0217 is another interesting piece of this peculiar puzzle.

Since the C IV $\lambda \lambda 1548,1550$ doublet is expected to be produced as a combination of nebular, stellar, and ISM/CGM components, we can use a simple combination of absorption and emission profiles to offer a possible model for the C IV profile observed in SL2S0217. Despite the absence of apparent C IV absorption, if we assume that the absorption in both C IV and Si IV is due to high ionization clouds in the surrounding ISM of the galaxy, we can use the average absorption profile (which is nearly identical to the Si IV $\lambda 1403$ profile; see Figure 7) to model the C IV absorption. Although the oscillator strength of Si IV $\lambda 1394$ is roughly two times larger than Si IV $\lambda 1403$, their relative absorption profile depths are closer to a factor of 1.5 , likely due to saturation. Similarly, C IV $\lambda 1548$ absorption is expected to be twice as strong as C IV $\lambda 1550$. Following the observed model of Si IV, we assume relative absorption profile strengths of $3: 2$ for both Si IV $\lambda \lambda 1394,1403$ and C IV $\lambda \lambda 1548,1550$. The total profile is then produced by co-adding the absorption profile with the emission predicted by the best photoionization model, $n \times F_{\lambda}$, where $n$ is an arbitrary scaling of the emission to best-match the observed spectrum of SL2S0217. The resulting model profiles and their components are plotted against the SL2S0217 spectrum in Figure 15. These model fits offer $a$ solution in which the Si IV and C IV observed profiles could be produced, but we caution that this interpretation cannot be confirmed.

\subsubsection{Nebular He II $\lambda 1640$}

Arguably the most aberrant feature of the SL2S0217 UV spectrum is the impressive nebular He II emission strength. Nebular He II emission is produced by recombination from the $\mathrm{He}^{+2}$ ionization state, and so requires very hard ionizing radiation with photons at energies $\geq 54 \mathrm{eV}$ in order to ionize $\mathrm{He}^{++}$. The interpretation of He II emission is further complicated by the fact that it can have both nebular and stellar origins. Stellar He II is produced by the stellar winds of massive stars, such as Wolf-Rayet (W-R) stars, and so is broadened in relation to the wind velocity (e.g., Brinchmann et al. 2008, Erb et al. 2010: Cassata et al. 2013).

Leitherer et al. (1999) showed that the number of W-R stars relative to $\mathrm{O}$ stars peaks around $10^{6.6} \mathrm{yrs}$, and so stellar winds may no longer be prominently contributing to the He II emission at a star-burst age of $10^{7} \mathrm{yrs}$, in line with the fact that we do not observe a significant broad component to the He II emission in SL2S0217. Further, Eldridge \& Stanway (2009) demonstrated that the WR He II $\lambda 1640$ feature is only prominent in binary models with stellar metallicities of $Z_{\star}=0.004$ or 0.008 , which
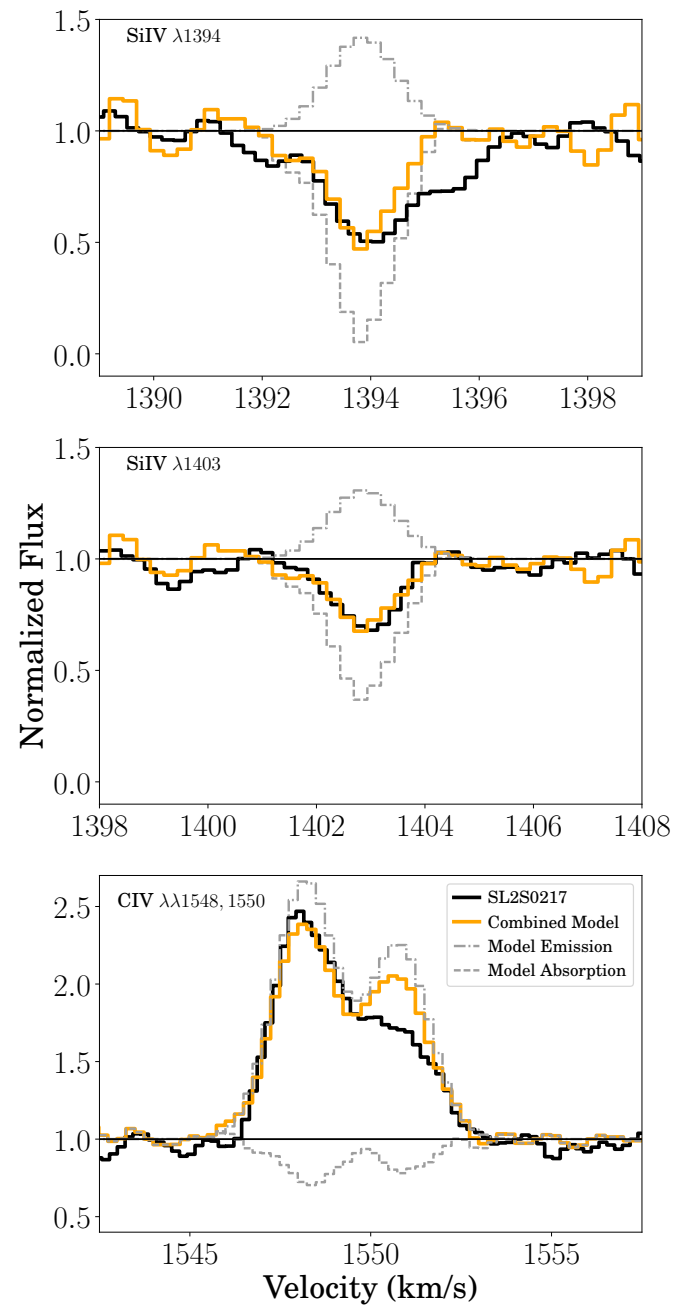

FIG. 15.- Model to produce the observed Si IV absorption and C IV emission features by combining scaled versions of the average absorption profile and the emission line ratios predicted by the photoionization described in Section 8.1.1

do not pair well with the gas-phase oxygen abundance of SL2S0217 $\left(Z_{n e b} \sim 0.001\right)$. Instead, the He II emission in SL2S0217 appears to be nebular in origin. In the photoionization models of Jaskot \& Ravindranath (2016), the narrow, nebular He II emission seen in SL2S0217 only becomes strong for more metal-rich systems during the W-R phase or when ionized by shocks. Other sources of high energy photons may be required to produce strong nebular He II, such as AGN, exotic Pop III stars, or highmass X-ray binaries (e.g., Shirazi \& Brinchmann 2012, Kehrig et al. 2015).

As discussed in Section 8.3.1, we dismiss a significant contribution to the ionizing budget from shocks as the models significantly overpredict the $\mathrm{O}$ III]/C III] budget. In addition to overshooting $\mathrm{O}$ III]/C III], the AGN models $(\S 8.3 .2)$ also produce an excess of $\mathrm{C}$ IV relative to $\mathrm{C}$ III] emission, but this argument is not definitive as some of the emission may be obscured by overlapping C IV absorption. However, AGN typically have large He II EWs ( 8Å; Hainline et al. 2011; Cassata et al. 2013), which are generally greater than those measured 
for $\mathrm{z} \sim 2$ galaxies (Scarlata et al. 2009, Erb et al. 2010) and for SL2S0217 $\left(\mathrm{EW}\left(\mathrm{He} \mathrm{II}_{n}\right)=2.4 \AA\right.$ or EW(He $\left.\mathrm{II}_{t o t}\right)$ $=2.8 \AA$, where the difference is due to the difficulty in deblending the narrow and wide components).

Photoionization by AGN has also been investigated using diagnostic plots of the $\mathrm{C}$ IV/He II and $\mathrm{C}$ III]/He II line ratios, both of which are sensitive to metallicity and ionization parameter (Villar-Martin et al. 1997). In Figure 16 we plot our stellar photoionization models described in Section 8.1.1 in comparison to photoionization from pure shocks and AGN for the C IV/He II versus C III]/He II ratios. The observations for SL2S0217 (black star), are extended to larger values (shaded region) in case C IV is underestimated (due to overlapping $\mathrm{C}$ IV absorption) and/or He II is overestimated (due to a stellar contribution), clearly suggesting a stellar origin of the photoionization budget. Note, however, that the stellar photoionization models still fail to reproduce the absolute strength / EW of the He II emission. Motivated by the uncertainties of these line ratios, we will present a preferable analysis in a forthcoming paper, that allows us to distinguish between sources of ionizing radiation using line ratios that are not complicated by stellar and CGM components.

In observations of star-forming galaxies, He II emission is commonly attributed to W-R stars, which typically show strong and broad $(\Delta \mathrm{v}>1000 \mathrm{~km} / \mathrm{s}$; Crowther (2007)) emission lines produced by their fast stellar winds. For example, Chandar et al. (2004) measured an extreme He II $\lambda 1640 \mathrm{EW}$ in the local Universe from W$\mathrm{R}$ stars in the massive cluster NGC3125-1 with a broad width of $\sim 1000 \mathrm{~km} \mathrm{~s}^{-1}$, and further supported by strong detections of the N IV $\lambda 1488$ and N v $\lambda 1720$ WN W-R features. We therefore rule out $\mathrm{W}$-R stars as the source of the He II emission on the account that we do not observe significant broad He II emission in SL2S0217, or other indicative feature, and that the $\mathrm{W}-\mathrm{R}$ phase occurs in a very short and early window that is unlikely the period of our observations. In binary star models, where the effects of mixing and mass transfer extends the life of these massive stars, He II emission peaks $\sim 50$ Myrs after a burst of $\mathrm{Z}_{\star}=0.004$ star formation. However, the He II emission from W-Rs depends on the metallicity, and the lower metallicity stellar spectra representative of SL2S0217 are not capable of reproducing the observed He II (see Figure 13 .

Theoretically, gravitational cooling radiation from gas accretion is another mechanism that can produce He II (as well as Ly $\alpha$ ) emission profiles like those in SL2S0217. In these models, infalling gas onto dark matter can be heated up to temperatures of $T \sim 10^{5} \mathrm{~K}$, and then is cooled by $\mathrm{H}$ and $\mathrm{He}$ line emission (Kereš et al. 2005 Yang et al. 2006). It has been suggested that Ly $\alpha$ halos (Ly $\alpha$ "blobs" that show extended Ly $\alpha$ emission on scales of tens of kpc or more, e.g., Caminha et al.|2016; Patrício et al. 2016) are the observational signature of this cooling radiation in forming galaxies (Haiman et al. 2000; Fardal et al. 2001). While inflowing gas would also help explain the strong blue peak in the Ly $\alpha$ profile, it is unlikely that SL2S0217 is a Ly $\alpha$ halo. Our observations and lensing model show a small galaxy with Ly $\alpha$ emission coming from three dense star-forming regions, where the Ly $\alpha$ emission extends beyond the continuum on much smaller spatial scales (as detected in narrow-band HST imaging; Erb et al. 2018, in prep.).

The current arsenal of stellar population models in the literature are only able to reproduce strong He II emission lines with pure photoionization for extreme stellar populations. Due to its high ionization potential, He II emission has been used as a probe to find Pop III stars (e.g., Visbal et al. 2015). A top-heavy IMF in extremely low metallicity regions can produce large He II line luminosities (Tumlinson \& Shull 2000, Schaerer 2003, Yoon et al. 2012). However, the He II EW is very sensitive to metallicity, and so requires $\mathrm{Z}<10^{-5}$ for this model. Steidel et al. (2016) predicted that H II regions with normal oxygen abundances can exhibit extreme UV features if they are powered by stars that are $\mathrm{O} / \mathrm{Fe}$ enhanced relative to solar. Such a scenario would be expected if the ISM was enriched by core-collapse SNe, and then the best-matching stellar model would actually have a metallicity several times lower than the gas-phase oxygen abundance. Since stellar spectra are primarily dependent on the total opacity, which is largely modulated by the Fe abundance (e.g., Rix et al. 2004), much harder ionizing radiation would be produced than is expected from the nebular metallicity. Alternatively, Tornatore et al. (2007) predicted that zero-metallicity regions can survive in low-density regions around large over densities, allowing Pop III stars to form, until as recently as $z \sim 2$.

Other studies do not require large modifications to relative abundances or metal-free stars to produce narrow He II emission. Kudritzki (2002) showed that certain $\mathrm{O}$ stars are indeed hot enough to ionize $\mathrm{He}$ II and Brinchmann et al. (2008) suggested that at low metallicities nebular He II is predominantly produced by $\mathrm{O}$ stars where optically thin winds can be penetrated by ionizing photons. The energy production of low-metallicity massive stars may also be increased if they are fast rotators (Meynet \& Maeder 2007). Further support is provided by the study of Kehrig et al. (2015), who found that the He II-ionization budget of $1 \mathrm{Z} \mathrm{w} 18$ can only be produced by models of either low-metallicity super-massive O stars or rotating metal-free stars. However, the details of very low-metallicity $\mathrm{O}$ stars are still widely debated, and further complicated by the fact that some He II nebulae don't appear to have surviving O or W-R stars (Kehrig et al. 2011). Gräfener \& Vink (2015) prosed an alternative scenario for the origin of narrow He II emission in which metal-poor, very massive stars form strong but slow W-R-type stellar winds due to their proximity to the Eddington limit. Post-AGB stars from an older stellar population may also produce He II emission. The combined radiation field of post-AGB stars will dominate the ionizing spectrum about 100 Myrs after a burst of star formation and would be strong enough to ionize He II (Binette et al. 1994). However, the He II luminosities produced by post-AGB stars are insufficient to explain the emission line strength seen for SL2S0217 (Cassata et al. 2013).

It seems likely that very massive stars play a significant role in the He II line strength. Unfortunately, the nature of metal-poor massive stars remains poorly understood and therefore very massive stars are often withheld from current population synthesis models. Theoretical 


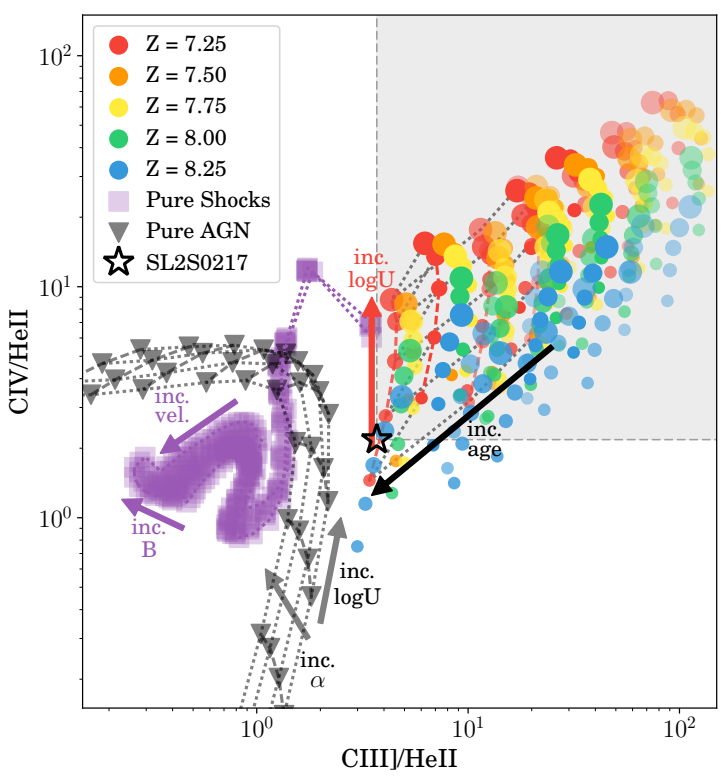

Fig. 16.- C IV/He II versus $\mathrm{C}$ III]/He II emission line diagnostic diagram. The stellar photoionization models described in Section 8.1 are shown as solid colored circles, where the metallicity increases with color, ionization parameter increases with point size, age increases with color saturation. As an example, for the CNSi $=$ 0.1 and $12+\log (\mathrm{O} / \mathrm{H})=7.25$ model, dashed-lines connect points of constant age and black dotted-lines connect points of constant ionization parameter. Pure shock and AGN models are also shown. The observed line ratios of SL2S0217 are depicted as the black star, where the gray shaded region indicates the possible values of SL2S0217 if C IV or He II are not purely nebular emission.

work on rotation and binary evolution of massive stars (e.g., Eldridge \& Stanway 2009; de Mink et al. 2014) has produced a vast range in the predicted lifetimes and energy output of low-metallicity massive stars. Further, these models make simplifying assumptions about the rotation velocities and distribution of binary separations that are poorly motivated. In the future, more sophisticated models of metal-poor massive stars may be able to explain the strength of high ionization nebular lines observed for SL2S0217. However, since narrow He II emission is more common at $z \sim 3$ (Cassata et al. 2013) than at $z \sim 0$ (Kehrig et al. 2011), it is possible that the stellar populations that produce He II ionizing radiation are more common at higher redshifts and are fundamentally different than typical populations in the local Universe.

\section{CONCLUSIONS}

We present the rest-frame UV Keck/LRIS spectrum of SL2SJ021737-051329 (SL2S0217), a lensed galaxy magnified by a factor of roughly 17 by a massive galaxy at $\mathrm{z}=0.65$. SL2S0217 is particularly interesting given its very low mass $\left(\mathrm{M}<10^{9} \mathrm{M}_{\odot}\right)$, low estimated metallically $\left(\mathrm{Z}_{\star} \sim 1 / 20 \mathrm{Z}_{\odot}\right)$, and extreme star-forming conditions that produce strong nebular UV emission lines in the absence of any apparent outflows. Because these characteristics are more common at higher redshifts (e.g., Brinchmann et al. 2008, Stark et al. 2014) and have been shown to be correlated with escaping ionizing radiation (e.g., Verhamme et al. 2017), we present SL2S0217 as a template to study the extreme conditions that may be responsible for the reionization-era.
In our analysis of the UV spectrum of SL2S0217 we found the Ly $\alpha$, interstellar absorption, and nebular emission features to be notable in the following ways:

1. The Ly $\alpha$ feature is observed purely in emission that is double-peaked and centered near the systemic velocity. The dominant blue peak and small peak separation indicate little-to-no outflowing neutral gas along the line of sight. Given the large Ly $\alpha$ EW, we measure an unexpectedly low Ly $\alpha$ escape fraction from the $L_{L y \alpha}^{o b s} / L_{L y \alpha}^{i n t}$ ratio of $7 \%$.

2. Nearly all of the observed absorption features are saturated due to optically thick ionized gas. However, we were able to use the weakest Si II and Fe II lines to estimate a relative abundance of $\log (\mathrm{Si} / \mathrm{Fe})=-0.225 \pm$ 0.118 , or $44-77 \%$ solar, where this underabundance can be accounted for by Si depletion onto dust grains. Similar to the Ly $\alpha$ emission, both the low- and high-ionization absorption features show profiles with nearly systemic velocity centers and small velocity ranges, indicating very little or no outflowing ionized gas along the sightline to the lensed galaxy.

3. Large equivalent widths are measured for the $\mathrm{C}$ IV $\lambda \lambda 1548,1550$, He II $\lambda 1640$, O III] $\lambda \lambda 1661,1666$, Si III] $\lambda \lambda 1883,1892$, and C III] $\lambda \lambda 1907,1909$ nebular emission lines. In the case of $\mathrm{C} \mathrm{III]}$ and $\mathrm{C} \mathrm{IV}$, these line strengths are similar to those observed for the extreme star-forming galaxies at higher redshifts $(z>7$; e.g., Stark et al. 2015, Stark 2016).

With the exception of He II, the relative emission line strengths can be reproduced by ionization from a very high ionization, low metallicity starburst with binaries. We rule out large contributions from AGN and shocks to the photoionization budget, suggesting that the emission features requiring the hardest radiation field likely result from extreme stellar populations that are beyond the capabilities of current models. We, therefore, argue that the combination of features observed for SL2S0217 are the result of an extreme episode of star formation in a young galaxy along a sightline that does not cross any outflowing gas.

We have studied the extreme spectral features of SL2S0217, finding both its absorption and emission features to be distinct from the general population of $z \sim$ $2-3$ galaxies. In particular, our models show that the emission line strengths of SL2S0217 require very hard ionizing radiation, and so it adds to the small population of galaxies with UV emission lines powered by the hard ionizing stellar spectra that may be responsible for the reionization era. Therefore, due to its optimal properties and leveraged by its lensed nature, SL2S0217 is an ideal template to study the extreme conditions that are important for reionization and thought to be more common in the early Universe.

This work evolved over the course of the project thanks to many creative and insightful conversations with colleagues, especially John Chisholm, Anne Jaskot, and Claudia Scarlata. We extend our gratitude to the Lorentz Center for useful discussions during the "Characterizing Galaxies with Spectroscopy with a view for JWST" workshop. Additionally, we are grateful to the referee for valuable comments that improved and clarified the paper. We wish to recognize and acknowledge 
the very significant cultural role and reverence that the summit of Mauna Kea has always had within the indigenous Hawaiian community. We are most fortunate to have the opportunity to conduct observations from this mountain.

DAB and DKE are supported by the US National Science Foundation through the Faculty Early Career Development (CAREER) Program, grant AST-1255591. Models in this work were computed using the computer cluster, Nemo, at The Leonard E Parker Center for Gravitation, Cosmology and Astrophysics is supported by NASA, the National Science Foundation, UWMilwaukee College of Letters and Science, and UWMilwaukee Graduate School. Any opinions, findings, and conclusions or recommendations expressed in this material are those of the author(s) and do not necessarily reflect the views of these organizations. 


\section{REFERENCES}

Allen, M. G., Groves, B. A., Dopita, M. A., Sutherland, R. S., \& Kewley, L. J. 2008, ApJS, 178, 20

Amorín, R., Fontana, A., Pérez-Montero, E., et al. 2017, Nature Astronomy, 1, 0052

Amorín, R., Grazian, A., Castellano, M., \& other. 2014, ApJ, 788, L4

Asplund, M., Grevesse, N., Sauval, A. J., \& Scott, P. 2009, ARA\&A, 47, 481

Baldassare, V. F., Reines, A. E., Gallo, E., \& Greene, J. E. 2015, ApJ, 809, L14

-. 2017, ApJ, 836, 20

Baldwin, J. A., Phillips, M. M., \& Terlevich, R. 1981, PASP, 93, 5

Bayliss, M. B., Rigby, J. R., Sharon, K., et al. 2014, ApJ, 790, 144

Behrens, C. \& Braun, H. 2014, A\&A, 572, A74

Berg, D. A., Skillman, E. D., Croxall, K. V., Pogge, R. W. Moustakas, J., et al. 2015, ApJ, 806, 16

Berg, D. A., Skillman, E. D., Henry, R. B. C., Erb, D. K., \& Carigi, L. 2016, ApJ, 827, 126

Berg, D. A., Skillman, E. D., Marble, A. R., et al. 2012, ApJ, 754, 98

Binette, L., Magris, C. G., Stasińska, G., \& Bruzual, A. G. 1994, A\&A, 292, 13

Brammer, G. B., Sánchez-Janssen, R., Labbé, I., et al. 2012, ApJ 758, L17

Brinchmann, J., Kunth, D., \& Durret, F. 2008, A\&A, 485, 657

Byler, N., Dalcanton, J. J., Conroy, C., \& Johnson, B. D. 2017 , ApJ, 840, 44

Calzetti, D., Armus, L., Bohlin, R. C., et al. 2000, ApJ, 533, 682

Caminha, G. B., Karman, W., Rosati, P., \& other. 2016, A\&A, 595, A100

Cardelli, J. A., Clayton, G. C., \& Mathis, J. S. 1989, ApJ, 345, 245

Cassata, P., Le Fèvre, O., Charlot, S., \& others. 2013, A\&A, 556, A68

Chandar, R., Leitherer, C., \& Tremonti, C. 2004, ApJ, 604, 153c

Choi, J., Conroy, C., \& Byler, N. 2017, ApJ, 838, 159

Christensen, L., Laursen, P., Richard, J., et al. 2012, MNRAS, 427, 1973

Christensen, L., Møller, P., Fynbo, J. P. U., \& Zafar, T. 2014 MNRAS, 445, 225

Cooray, A., Fu, H., Calanog, J., \& others. 2011, ArXiv e-prints

Cowie, L. L., Barger, A. J., \& Hu, E. M. 2011, ApJ, 738, 136

Crowther, P. A. 2007, ARA\&A, 45, 177

Croxall, K. V., Pogge, R. W., Berg, D. A., Skillman, E. D., \& Moustakas, J. 2015, ApJ, 808, 42

-. 2016, ApJ, 830, 4

de Barros, S., Vanzella, E., Amorín, R., \& others. 2016, A\&A, $585, \mathrm{~A} 51$

de Mink, S. E., Sana, H., Langer, N., Izzard, R. G., \& Schneider, F. R. N. 2014, ApJ, 782, 7

De Robertis, M. M., Dufour, R. J., \& Hunt, R. W. 1987, JRASC, 81,195

Dessauges-Zavadsky, M., D'Odorico, S., Schaerer, D., et al. 2010, A\&A, 510, A26

Dijkstra, M. 2014, PASA, 31, e040

Dijkstra, M., Haiman, Z., \& Spaans, M. 2006, ApJ, 649, 37

Du, X., Shapley, A. E., Martin, C. L., \& Coil, A. L. 2017, ApJ, 838,63

Eldridge, J. J. \& Stanway, E. R. 2009, MNRAS, 400, 1019

-. 2016, MNRAS, 462, 3302

Erb, D. K., Pettini, M., Shapley, A. E., et al. 2010, ApJ, 719, 1168

Erb, D. K., Quider, A. M., Henry, A. L., \& Martin, C. L. 2012, ApJ, 759, 26

Esteban, C., Bresolin, F., Peimbert, M., et al. 2009, ApJ, 700, 654

Esteban, C., García-Rojas, J., Carigi, L., et al. 2014, MNRAS, 443,624

Esteban, C., Peimbert, M., Torres-Peimbert, S., \& Rodríguez, M. 2002, ApJ, 581, 241

Fardal, M. A., Katz, N., Gardner, J. P., \& others. 2001, ApJ, 562 , 605

Feltre, A., Charlot, S., \& Gutkin, J. 2016, MNRAS, 456, 3354

Ferland, G. J., Porter, R. L., van Hoof, P. A. M., \& others. 2013, Rev. Mexicana Astron. Astrofis., 49, 137

Finkelstein, S. L., Papovich, C., Ryan, R. E., et al. 2012, ApJ, 758,93

Fontanot, F., Cristiani, S., Pfrommer, C., Cupani, G., \& Vanzella, E. 2014, MNRAS, 438, 2097

Fosbury, R. A. E., Villar-Martín, M., \& others. 2003, ApJ, 596, 797

Fruchter, A. S., Hack, W., Dencheva, N., Droettboom, N., \& Greenfield, P. 2010, in 2010 Space Telescope Science Institute Calibration Workshop, p. 382-387, 382-387

García-Rojas, J. \& Esteban, C. 2007, ApJ, 670, 457

Garnett, D. R. 1992, A.J, 103, 1330
Garnett, D. R . Dufour, R. J., Peimbert, M., \& others. 1995a, ApJ, 449, L77

Garnett, D. R., Shields, G. A., Peimbert, M., et al. 1999, ApJ, 513,168

Garnett, D. R., Skillman, E. D., Dufour, R. J., et al. 1995b, ApJ, 443,64

Gebhardt, K., Bender, R., Bower, G., et al. 2000, ApJ, 539, L13

Gould, A. \& Weinberg, D. H. 1996, ApJ, 468, 462

Gräfener, G. \& Vink, J. S. 2015, A\&A, 578, L2

Grogin, N. A., Kocevski, D. D., Faber, S. M., et al. 2011, ApJS, 197,35

Gronke, M., Dijkstra, M., McCourt, M., \& Oh, S. P. 2016, ApJ, 833, L26

Groves, B. A., Dopita, M. A., \& Sutherland, R. S. 2004, ApJS, 153,75

Gültekin, K., Richstone, D. O., Gebhardt, K., \& othes. 2009, ApJ, 698, 198

Gutkin, J., Charlot, S., \& Bruzual, G. 2016, MNRAS, 462, 1757

Haiman, Z., Spaans, M., \& Quataert, E. 2000, ApJ, 537, L5

Hainline, K. N., Shapley, A. E., Greene, J. E., \& Steidel, C. C. 2011, ApJ, 733, 31

Hainline, K. N., Shapley, A. E., Kornei, K. A., Pettini, M., Buckley-Geer, E., et al. 2009, ApJ, 701, 52

Hashimoto, T., Verhamme, A., Ouchi, M., et al. 2015, ApJ, 812, 157

Henry, A., Berg, D., Scarlata, C., Verhamme, A., \& Erb, D. 2018 ApJ, submitted

Henry, A., Scarlata, C., Martin, C. L., \& Erb, D. 2015, ApJ, 809, 19

Hummer, D. G. \& Storey, P. J. 1987, MNRAS, 224, 801

Humphreys, E. M. L., Reid, M. J., Greenhill, L. J., Moran, J. M., \& Argon, A. L. 2008, ApJ, 672, 800

Izotov, Y. I., Schaerer, D., Thuan, T. X., \& others. 2016, MNRAS, 461, 3683

James, B. L., Pettini, M., Christensen, L., et al. 2014, MNRAS, 440,1794

Jaskot, A. E. \& Oey, M. S. 2013, ApJ, 766, 91

Jaskot, A. E., Oey, M. S., Scarlata, C., \& Dowd, T. 2017, ApJ, 851, L9

Jaskot, A. E. \& Ravindranath, S. 2016, ApJ, 833, 136

Jenkins, E. B. 2009, ApJ, 700, 1299

Jenkins, E. B. \& Wallerstein, G. 2017, ApJ, 838, 85

Jones, T., Stark, D. P., \& Ellis, R. S. 2012, ApJ, 751, 51

Jones, T. A., Ellis, R. S., Schenker, M. A., \& Stark, D. P. 2013, ApJ, 779, 52

Kehrig, C., Oey, M. S., Crowther, P. A., \& others. 2011, A\&A, $526, \mathrm{~A} 128$

Kehrig, C., Vílchez, J. M., Pérez-Montero, E., \& others. 2015, ApJ, 801, L28

Kereš, D., Katz, N., Weinberg, D. H., \& Davé, R. 2005, MNRAS, 363,2

Kewley, L. J. \& Dopita, M. A. 2002, ApJS, 142, 35

Kobayashi, C., Umeda, H., Nomoto, K., Tominaga, N., \& Ohkubo, T. 2006, ApJ, 653, 1145

Koekemoer, A. M., Faber, S. M., Ferguson, H. C., et al. 2011, ApJS, 197, 36

Kormendy, J. \& Ho, L. C. 2013, ARA\&A, 51, 511

Kornei, K., Shapley, A., Erb, D., et al. 2010, ApJ, 711, 693

Kornei, K. A., Shapley, A. E., Martin, C. L., et al. 2013, ApJ, 774,50

Krabbe, A. C., Rosa, D. A., Dors, O. L., Pastoriza, M. G., \& others. 2014, MNRAS, 437, 1155

Kudritzki, R. P. 2002, ApJ, 577, 389

Kulas, K. R., Shapley, A. E., Kollmeier, J. A., et al. 2012, ApJ, 745,33

Labbé, I., Bouwens, R., Illingworth, G. D., \& Franx, M. 2006, ApJ, 649, L67

Leitherer, C., Leão, J. R. S., Heckman, T. M., \& others. 2001, ApJ, 550, 724

Leitherer, C., Schaerer, D., Goldader, J. D., et al. 1999, ApJS, 123,3

Leitherer, C., Tremonti, C. A., Heckman, T. M., \& Calzetti, D. 2011, AJ, 141, 37

López-Sánchez, Á. R., Esteban, C., García-Rojas, J., Peimbert, M., \& Rodríguez, M. 2007, ApJ, 656, 168

Luridiana, V., Morisset, C., \& Shaw, R. A. 2012, in IAU Symposium, Vol. 283, IAU Symposium, 422-423

Luridiana, V., Morisset, C., \& Shaw, R. A. 2015, A\&A, 573, A42

Madau, P. \& Haardt, F. 2015, ApJ, 813, L8

Mainali, R., Kollmeier, J. A., Stark, D. P., \& others. 2017, ApJ, 836, L14

Mas-Ribas, L. \& Dijkstra, M. 2016, ApJ, 822, 84

Masters, D., Faisst, A., \& Capak, P. 2016, ApJ, 828, 18

Masters, D., McCarthy, P., Siana, B., et al. 2014, ApJ, 785, 153 
Matsuoka, K., Nagao, T., Maiolino, R., Marconi, A., \& Taniguchi, Y. 2009, A\&A, 503, 721

Matteucci, F. \& Greggio, L. 1986, A\&A, 154, 279

Meynet, G. \& Maeder, A. 2007, A\&A, 464, L11

Morton, D. C. 2003, ApJS, 149, 205

Moustakas, J. \& Kennicutt, Jr., R. C. 2006, ApJS, 164, 81

Oke, J. B., Cohen, J. G., Carr, M., et al. 1995, PASP, 107, 375

Osterbrock, D. E. 1989, Astrophysics of gaseous nebulae and active galactic nuclei

Patrício, V., Richard, J., Verhamme, A., et al. 2016, MNRAS, 456, 4191

Pellegrini, E. W., Oey, M. S., Winkler, P. F., \& others. 2012, ApJ, 755, 40

Pequignot, D., Petitjean, P., \& Boisson, C. 1991, A\&A, 251, 680

Pettini, M. \& Cooke, R. 2012, in Nuclei in the Cosmos (NIC XII), 71

Pettini, M., Ellison, S. L., Bergeron, J., \& Petitjean, P. 2002a, A\&A, 391, 21

Pettini, M., Rix, S. A., Steidel, C. C., et al. 2002b, ApJ, 569, 742

Pettini, M., Steidel, C. C., Adelberger, K. L., Dickinson, M., \& Giavalisco, M. 2000, ApJ, 528, 96

Pilyugin, L. S. \& Thuan, T. X. 2005, ApJ, 631, 231

Quider, A. M., Pettini, M., Shapley, A. E., \& Steidel, C. C. 2009, MNRAS, 398, 1263

Quider, A. M., Shapley, A. E., Pettini, M., Steidel, C. C., \& Stark, D. P. 2010, MNRAS, 402, 1467

Reddy, N. A., Pettini, M., Steidel, C. C., et al. 2012, ApJ, 754, 25

Reddy, N. A., Steidel, C. C., Pettini, M., \& others. 2008, ApJS, 175,48

Reines, A. E., Plotkin, R. M., Russell, T. D., \& others. 2014, ApJ, 787, L30

Reines, A. E., Sivakoff, G. R., Johnson, K. E., \& Brogan, C. L. 2011, Nature, 470, 66

Rich, J. A., Kewley, L. J., \& Dopita, M. A. 2014, ApJ, 781, L12

Rigby, J. R., Bayliss, M. B., Chisholm, J., et al. 2017, ArXiv e-prints

Rigby, J. R., Wuyts, E., Gladders, M. D., Sharon, K., \& Becker, G. D. 2011, ApJ, 732, 59

Rix, S. A., Pettini, M., Leitherer, C., et al. 2004, ApJ, 615, 98

Rubin, K. H. R., Prochaska, J. X., Ménard, B., et al. 2011, ApJ, 728,55

Sanders, R. L., Shapley, A. E., Kriek, M., \& others. 2016, ApJ, 816,23

Scarlata, C., Colbert, J., Teplitz, H. I., \& others. 2009, ApJ, 706, 1241

Scarlata, C. \& Panagia, N. 2015, ApJ, 801, 43

Schaerer, D. 2003, A\&A, 397, 527

Schlafly, E. F. \& Finkbeiner, D. P. 2011, ApJ, 737, 103

Schwartz, C. M., Martin, C. L., Chandar, R., \& others. 2006, 646 858

Senchyna, P., Stark, D. P., Vidal-García, A., et al. 2017, MNRAS, 472, 2608

Shapley, A. E. 2011, ARA\&A, 49, 525

Shapley, A. E., Reddy, N. A., Kriek, M., et al. 2015, ApJ, 801, 88

Shapley, A. E., Steidel, C. C., Pettini, M., \& Adelberger, K. L. 2003, ApJ, 588, 65

Shirazi, M. \& Brinchmann, J. 2012, MNRAS, 421, 1043

Smit, R., Swinbank, A. M., Massey, R., Richard, J., Smail, I., \& Kneib, J.-P. 2017, MNRAS, 467, 3306
Spitzer, Jr., L. 1978, JRASC, 72, 349

Stanway, E. R., Eldridge, J. J., \& Becker, G. D. 2016, MNRAS, 456,485

Stark, D. P. 2016, ARA\&A, 54, 761

Stark, D. P., Richard, J., Charlot, S., \& others. 2015, MNRAS, 450, 1846

Stark, D. P., Richard, J., Siana, B., et al. 2014, MNRAS, 445 , 3200

Steidel, C. C., Erb, D. K., Shapley, A. E., Pettini, M., et al. 2010, ApJ, 717, 289

Steidel, C. C., Pettini, M., \& Adelberger, K. L. 2001, ApJ, 546, 665

Steidel, C. C., Rudie, G. C., Strom, A. L., et al. 2014, ApJ, 795, 165

Steidel, C. C., Strom, A. L., Pettini, M., Rudie, G. C., Reddy,

N. A., \& Trainor, R. F. 2016, ApJ, 826, 159

Timmes, F. X., Woosley, S. E., \& Weaver, T. A. 1995, ApJS, 98, 617

Tolstoy, E., Hill, V., \& Tosi, M. 2009, ARA\&A, 47, 371

Tornatore, L., Ferrara, A., \& Schneider, R. 2007, MNRAS, 382, 945

Trainor, R. F., Steidel, C. C., Strom, A. L., \& Rudie, G. C. 2015, ApJ, 809, 89

Trainor, R. F., Strom, A. L., Steidel, C. C., \& Rudie, G. C. 2016 , ApJ, 832, 171

Tu, H., Gavazzi, R., Limousin, M., Cabanac, R., Marshall, P. J., Fort, B., Treu, T., Péllo, R., Jullo, E., Kneib, J.-P., \& Sygnet, J.-F. 2009, A\&A, 501, 475

Tumlinson, J. \& Shull, J. M. 2000, ApJ, 528, L65

van Zee, L. \& Haynes, M. 2006, ApJ, 636, 214

Vanden Berk, D. E., Richards, G. T., Bauer, A., \& others. 2001 AJ, 122,549

Vanzella, E., Balestra, I., Gronke, M., \& others. 2017, MNRAS, 465,3803

Vanzella, E., De Barros, S., Cupani, G., \& others. 2016, ApJ, 821, L27

Vegetti, S. \& Koopmans, L. V. E. 2009, MNRAS, 392, 945

Verhamme, A., Orlitová, I., Schaerer, D., \& Hayes, M. 2015, A\&A, 578, A7

Verhamme, A., Orlitová, I., Schaerer, D., et al. 2017, A\&A, 597, A13

Verhamme, A., Schaerer, D., \& Maselli, A. 2006, A\&A, 460, 397

Villar-Martin, M., Tadhunter, C., \& Clark, N. 1997, A\&A, 323, 21

Visbal, E., Haiman, Z., \& Bryan, G. L. 2015, MNRAS, 450, 2506

Whitaker, K. E., van Dokkum, P. G., Brammer, G., \& Franx, M. 2012, ApJ, 754, L29

Wise, J. H., Demchenko, V. G., Halicek, M. T., et al. 2014 , MNRAS, 442, 2560

Wright, E. L. 2006, PASP, 118, 1711

Yamada, T., Matsuda, Y., Kousai, K., et al. 2012, ApJ, 751, 29

Yang, Y., Decarli, R., Dannerbauer, H., et al. 2012, ApJ, 744, 178

Yang, Y., Zabludoff, A. I., Davé, R., \& ohter. 2006, ApJ, 640, 539

Yoon, S.-C., Dierks, A., \& Langer, N. 2012, A\&A, 542, A113

Yuan, T.-T. \& Kewley, L. J. 2009, ApJ, 699, L161 